University of Louisville

ThinkIR: The University of Louisville's Institutional Repository

$5-2017$

\title{
Synthesis and fundamental property studies of energy material under high pressure.
}

Meysam Akhtar

University of Louisville

Follow this and additional works at: https://ir.library.louisville.edu/etd

Part of the Condensed Matter Physics Commons

\section{Recommended Citation}

Akhtar, Meysam, "Synthesis and fundamental property studies of energy material under high pressure." (2017). Electronic Theses and Dissertations. Paper 2621.

https://doi.org/10.18297/etd/2621

This Doctoral Dissertation is brought to you for free and open access by ThinkIR: The University of Louisville's Institutional Repository. It has been accepted for inclusion in Electronic Theses and Dissertations by an authorized administrator of ThinkIR: The University of Louisville's Institutional Repository. This title appears here courtesy of the author, who has retained all other copyrights. For more information, please contact thinkir@louisville.edu. 


\title{
SYNTHESIS AND FUNDAMENTAL PROPERTY STUDIES OF ENERGY MATERIAL UNDER HIGH PRESSURE
}

\author{
By \\ Meysam Akhtar \\ B.S., Shahid Beheshti University, 2007 \\ M.S., University of Louisville, 2013 \\ A Dissertation \\ Submitted to the Faculty of the \\ College of Arts and Sciences of the University of Louisville \\ in Partial Fulfillment of the Requirements \\ for the Degree of \\ Doctor of Philosophy \\ in Physics \\ Department of Physics and Astronomy \\ University of Louisville \\ Louisville, Kentucky
}

May 2017 
Copyright 2002 by Meysam Akhtar

All rights reserved 



\title{
SYNTHESIS AND FUNDAMENTAL PROPERTY STUDIES OF ENERGY MATERIAL UNDER HIGH PRESSURE
}

\author{
By \\ Meysam Akhtar \\ B.S., Shahid Beheshti University, 2007 \\ M.S., University of Louisville, 2013 \\ A Dissertation Approved on
}

April 19, 2017

by the following Dissertation Committee:

Dr. Gamini Sumanasekera

Dr. Jacek B. Jasinski

Dr. Chakram S. Jayanthi

Dr. Ming Yu

Dr. Jinjun Liu 


\section{ACKNOWLEDGMENTS}

I would like to express my deepest appreciation and thanks to both of my advisors Dr. Gamini Sumanasekera, and Dr. Jacek Jasinski. I am grateful to Dr. Gamini for his great support and guidance throughout my entire $\mathrm{PhD}$ program. In science, he is exceptionally knowledgeable and has a great drive for developing homemade equipment and experimental setup as they become required in a project. I am also thankful to Dr. Jasinski whose invaluable mentoring and encouragement were vital to the completion of this dissertation. I have been inspired by his efforts in contributing to the scientific community. I would like to acknowledge my Ph.D. committee members Dr. Chakram S. Jayanthi, Dr. Ming Yu, and Dr. Jinjun Liu for their valuable comments and suggestions. My particular thanks go to Dr. Jayanthi, Chair of the Department of Physics and Astronomy, for the discussions about my research work and $\mathrm{PhD}$ career in many occasions. I also wish to express my gratitude to Dr. Chris Davis, Graduate Program Director who is a great resource for graduate students in UofL Physics Department, and his advice was essential in achieving my academic goals.

My thanks also go to Dr. Mahendra Sunkara, Director of the Conn Center for Renewable Energy Research, who gave me the opportunity to be a member of the ConnCenter family. I have received generous financial support from Conn Center during my graduate studies. I would also like to thank Dr. Andriy Durygin, who trained me in highpressure experimental methods using diamond anvil cell in CeSMEC at Florida 
International University. Dr. Madhu Menon performed the theoretical DFT calculations required in my work. I am thankful for his contribution and insightful comments. I am also grateful to Ms. Afsar Safaeian, my high school mathematics teacher. Every encounter with math is a reminder of her great efforts in teaching me. I would like to acknowledge the support from School of Interdisciplinary and Graduate Studies through "doctoral dissertation completion award" and the support from "John Dillon Jr Physics Fellowship". I am indebted to administrative and technical staff from Physics Department, and Conn Center, Mary Gayle Wrocklage, Rea Diehlmann, Eunice De Lourdes Salazar, Andrew Marsh, Rodica McCoy, and Joshua Rimmer for their assistance.

I was lucky to work with wonderful research associates and post-doctoral fellows during my research. I wish to express a sincere thank you to Dr. Bijandra Kumar, Dr. Ramchandra K. Rao, Dr. John Samuel Dilip Jangam, Dr. Dominika Ziolkowska, and Dr. Ruvini Dharmadasa. I am thankful to my former and current labmates, George Anderson, Ruwantha Jayasinghe, Venkat Kalyan Vendra, Swathi Sunkara, Ruchira Dharmasena, Rong Zhao, Sahar Pishgar, and Brandon Lavery.

My deepest gratitude and love to Farzaneh Hoveyda, my love, life partner, and soulmate who has been a constant source of encouragement and support. She also helped me in writing this dissertation through great discussion about various scientific aspects. I am heartily thankful to my parents, Faramarz, Soroor, and my brother, Misagh, for tremendous emotional support. 


\title{
ABSTRACT \\ SYNTHESIS AND FUNDAMENTAL PROPERTY STUDIES OF ENERGY MATERIAL UNDER HIGH PRESSURE \\ Meysam Akhtar \\ April 19, 2017
}

\begin{abstract}
Recently, high-pressure science and technology has flourished and rapidly advanced to impact a wide domain of materials and physical sciences. One of the most substantial technological developments is the integration of samples at ultrahigh pressure with a wide range of in-situ probing techniques. Applications of extreme pressure have significantly enriched our understanding of the electronic, phonon, and doping effects on the newly emerged two-dimensional (2D) materials. Under high pressure, materials' atomic volume radically decreases, and electronic density rises, which will lead to extraordinary chemical reaction kinetic and mechanisms. The promising capability of high pressure combine with the significance of novel emerging 2D materials in energyrelated research was the main motivation of this dissertation.
\end{abstract}

Firstly, the application of high pressure to enable the direct synthesis of $\alpha$ $\mathrm{AgGaO}_{2}$ through a reaction of $\mathrm{Ag}_{2} \mathrm{O}$ and $\mathrm{Ga}_{2} \mathrm{O}_{3}$ is demonstrated. The synthesized samples were extensively characterized, and their crystal phase and chemical composition were confirmed. Especially, the rhombohedral delafossite crystal phase of the prepared sample was verified by electron diffraction. The vibrational phonon modes 
were investigated using a combination of ab initio density functional theory (DFT) and experimental Raman measurement. In addition, using a modified DFT to calculate the electronic band structure of $\alpha-\mathrm{AgGaO}_{2}$ reported a more accurate valu of theoretical ${ }^{1}$ band gap than those have been reported previously.

Two-dimensional (2D) materials with efficient ion transport between the layers and the large surface areas have demonstrated promise for various energy-related applications. Few-layer black phosphorus (phosphorene), as a novel two-dimensional (2D) material, is gaining researchers' attention due to the exceptional properties, including puckered layer structure, widely tunable band gap, strong in-plane anisotropy, and high carrier mobility. Phosphorene application expanded from energy storage and conversion devices to thermoelectrics, optoelectronic and spintronic to sensors and actuators. Several recent theoretical studies have indicated that strain engineering can be a viable strategy to tune the electronic structure of phosphorene. Although several theoretical studies have predicted an electronic phase transition such as direct-indirect bandgap and semiconductor-metal transitions, there is not experimental study to indicate the transition.

Next, in this dissertation, a systematic experimental study of in situ high-pressure Raman and PL spectroscopy of phosphorene was reported. Furthermore, short transport growth of bulk black phosphorus and also, liquid-phase exfoliation technique to preparing few-layer black phosphorus was described. The study motivated by a better understanding of high-pressure effects on optical properties and band structure of this

\footnotetext{
${ }^{1}$ Performed by Dr. Madhu Menon (Center for Computational Sciences at the University of Kentucky)
} 
material system. This study help to verify theoretical predictions and to enhance fundamental understanding of relationships between strain and electronic band structure, enabling rational strain engineering towards additional functionalities and device applications of phosphorene and few-layer BP. In situ characterization techniques are invaluable for a fundamental understanding of materials, their processing, and functionalities.

Three-dimensional architecture of graphene has also attracted considerable attentions as an effective way to utilize the unique inherent properties of graphene sheets in practical applications. Three-dimensional graphene-based materials offer an easy and versatile platform for functionalization and integration into devices. Furthermore, the interlocking of graphene sheets into 3D structures solve the restacking issue and make 3D graphene-based materials more compatible with conventional material processing.

Finally, in the dissertation, we report a novel, inexpensive, and highly scalable, approach of fabricating a three-dimensional graphene network (foam) via pyrolysis of organic materials as the source of carbon. A template-assisted method to prepare and tune the properties of a high-quality $3 \mathrm{D}$ graphene network was described. In this simple method, the 3D graphene foam is synthesized in a controlled environment by thermal decomposition of the organic materials in the presence of $\mathrm{Ni}$ foam which plays a dual role of catalyst and 3D template. This technique can efficiently facilitate and control the in situ nitrogen doping of 3D graphene structure by adjusting the growth parameters and choosing the right organic materials (i.e. nitrogen-containing organic acids). In this work, inexpensive organic materials including caffeine $\left(\mathrm{C}_{8} \mathrm{H}_{10} \mathrm{~N}_{4} \mathrm{O}_{2}\right)$, urea $\left(\mathrm{CH}_{4} \mathrm{~N}_{2} \mathrm{O}\right)$ and acetaminophen $\left(\mathrm{C}_{8} \mathrm{H}_{9} \mathrm{NO}_{2}\right)$ were used with a citric acid solution as the source of carbon 
and nitrogen. Nitrogenation of 3D graphene foam create an effective improvement of properties which is suitable for an extensive range of new energy and environmental applications. Our Raman analysis indicated an improvement of graphene network quality with an increase of synthesis temperature between $650^{\circ} \mathrm{C}$ and $1000{ }^{\circ} \mathrm{C}$. Both Raman and TEM study (HRTEM, SAED, and EELS) showed uniform coverage and high crystallinity of multilayered graphitic shells formed in samples synthesis at $1000{ }^{\circ} \mathrm{C}$. The motivation for this $3 \mathrm{D}$ graphene research is to use in-situ high-pressure measurements to study fundamental properties of these materials including its vibrational structures, doping and functionalization. With its distinct Raman signatures dependent on the quality and structure, defect distribution, as well types of dopants and their concertation, 3D graphene seems well-suited for high-pressure in-situ Raman studies. These type of measurements are proposed as part of the future, follow-up research. 


\section{TABLE OF CONTENTS}

PAGE

ACKNOWLEDGMENTS ............................................................................... ii

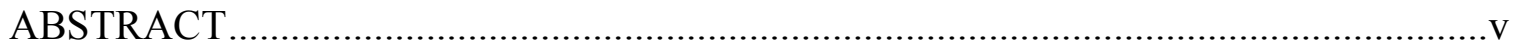

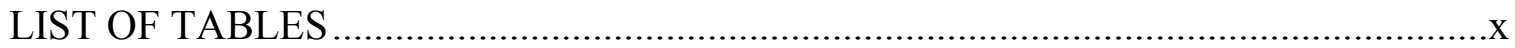

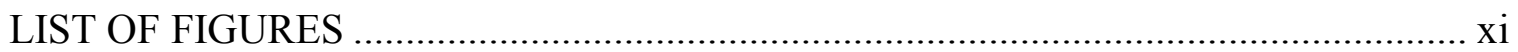

CHAPTER 1: BACKGROUND ....................................................................... 1

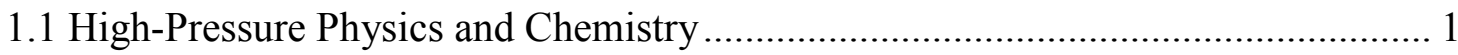

1.2 The Nobel Prizes for High-Pressure Research ................................................ 6

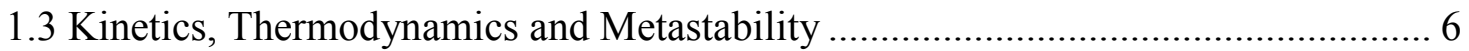

1.4 Semiconductors under High Pressure .............................................................. 7

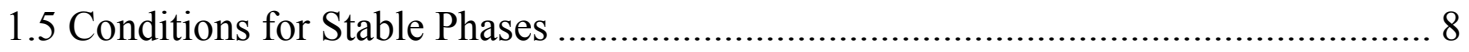

1.6 Rhombohedral $\alpha-\mathrm{AgGaO}_{2}\left(\right.$ Semiconductor Compounds with $\mathrm{ABC}_{2}$ Form) ......... 10

1.7 Three-Dimensional (3D) Graphene Architecture ........................................... 11

1.8 Few-Layer Black Phosphorus (Phosphorene) ............................................... 13

CHAPTER 2: EXPERIMENTAL METHODS ....................................................... 15

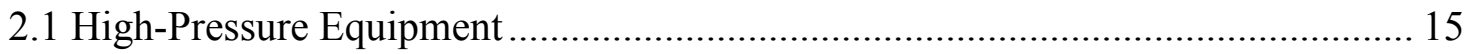

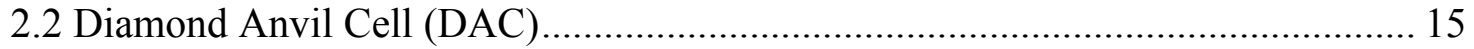




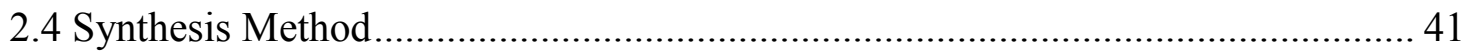

CHAPTER 3: RESULTS AND DISCUSSION ...................................................... 52

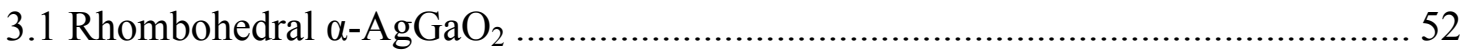

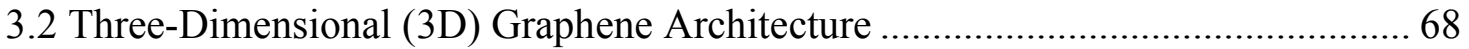

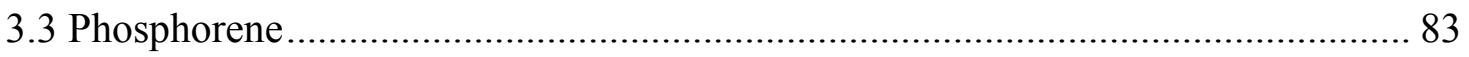

CHAPTER 4: SUMMARY AND OUTLOOK......................................................... 101

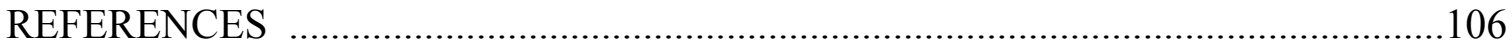

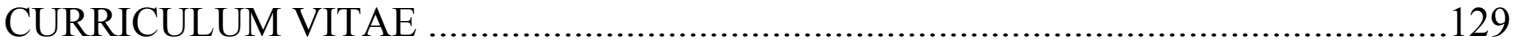




\section{LIST OF TABLES}

PAGE

Table 1.1 | In-situ High Pressure Probes with references. ............................................... 10

Table 2.1 | The Wavelength Ranges and Sources of the Radiation.................................. 30

Table 2.2 | List of samples prepared for this work....................................................... 43

Table $3.1 \mid$ Crystallographic Parameters Obtained for the DFT-Optimized $\alpha-\mathrm{AgGaO}_{2} \ldots 64$

Table 3.2 | Experimental and calculated Raman modes. ................................................ 67 


\section{LIST OF FIGURES}

PAGE

Figure $1.1 \mid$ Range of pressure in the universe and obtainable range in available laboratory

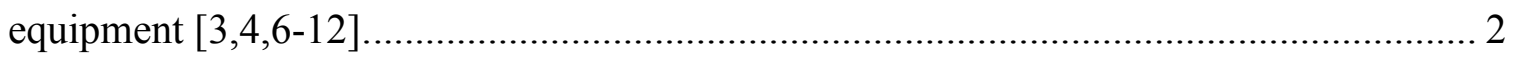

Figure 1.2 | High pressure impact on materials and its applications in materials science.. 3

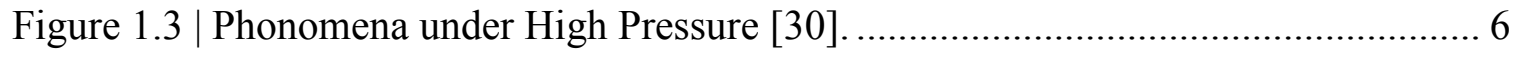

Figure 1.4 | Energy Profile Diagram of a Phase Transformation....................................... 7

Figure 2.1 | Schematics of the diamond anvil cell (DAC) and a photograph of our gas membrane driven DAC. Typical DAC is composed of two opposed diamond anvils with a metallic gasket in between providing a small chamber for the sample........................ 16

Figure 2.2 | Magnified Photograph of a Diamond Anvil under Optical Microscope. ...... 18

Figure 2.3 | Schematic of two diamond anvils with the standard design cut (Culet and

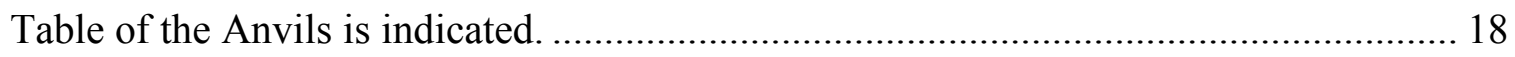

Figure 2.4 | Few Different Diamond Anvil Designs and Cuts that is commercially

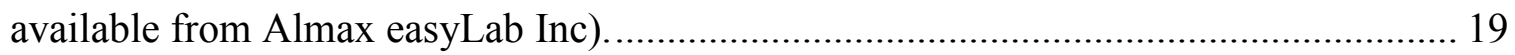

Figure 2.5 | Increasing the pressure inside the sample chamber by applying a force onto

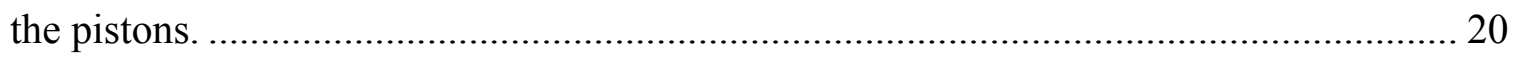

Figure 2.6 | Top View through Diamonds with Optical Microscope Showing the

Misalignment of Anvils. 
Figure 2.7 | Alignment of the diamond anvils and Loading Samples (Powder) in Gasket Hole (Sample Chamber) under the Microscope............................................................ 22

Figure 2.8| Small Hole at the Center of the Metallic Gasket as a Sample Chamber. ....... 23

Figure 2.9 | The Micro EDM System and Optical Zoom Microscope............................ 24

Figure 2.10 | Internal Resistive Heater DAC System and the External Furnace that we

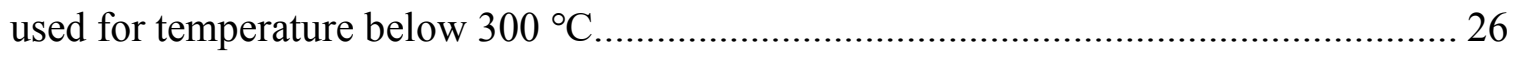

Figure 2.11 | Compact resistive heater $(80 \mathrm{~W})$, heater components, and DC power supply. 26

Figure 2.12 | Burned heater components due to excess of current into the metal resistor.27 Figure 2.13 | Right: Ruby Photoluminescence Spectrum undergo a Red-Shift by Increasing the Pressure. Right: The gas pressure in the membrane of the DAC can be calibrated to be a scale of the sample pressure. ........................................................ 28 Figure 2.14 | Top Row: SEM Images of Two different Ruby Chips and Photograph of Ruby Chips inside Gasket Hole. Bottom Row: The Gas Controller and Gas Membrane along with the DAC.

Figure 2.15 | The sketch shows different forms of energy exchange between a photon and the Sample 31

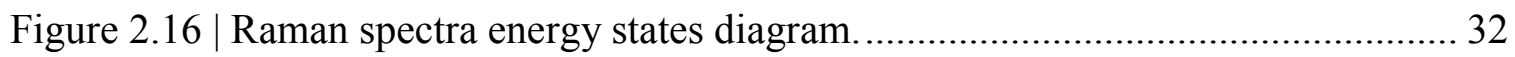

Figure 2.17 | Photoluminescence energy states diagram. ......................................... 33 Figure 2.18| Our modified Raman and PL system for in situ high-pressure measurements.

Figure 2.19 | Our developed optical setup for in situ high-pressure UV/Vis spectroscopy. 
Figure 2.20| Testing Optical Setup for In Situ High-Pressure UV/Vis Spectroscopy. Transmission Absorption Spectra of a Gasket Hole (200 $\mu \mathrm{m}$ in diameter) Covered by Orange Filter. 36

Figure 2.21 | Temperature-Pressure diagram representing conditions used in our DACbased $\alpha-\mathrm{AgGaO}_{2}$ synthesis experiments. Each square represents one experiment and its specific conditions. Green check mark denotes formation of rhombohedral $\alpha-\mathrm{AgGaO}_{2}$. Red crosses show experiments, which did not produce rhombohedral $\alpha-\mathrm{AgGaO}_{2} \ldots \ldots \ldots . . .44$ Figure 2.22 | Schematic image of single graphene sheet and graphite layers.

Figure 2.23 | Chemical Vapor Deposition System setup. It was used for both CVD and the developed pyrolysis methods. 45 Figure 2.24 | Optical and scanning electron microscopy (SEM) images of nickel foam. 46 Figure 2.25 | Photograph of three-dimensional graphene foams fabricated at 500, 650, 800 , and $1000{ }^{\circ} \mathrm{C}$ temperature and with different pre-treatment of nickel surface. 46 Figure 2.26 | Nickel foam (center), three-dimensional graphene before (left) and after (right) etching the nickel template.

Figure 2.27 | Molecular structure and chemical formula of organic precursors used for graphene growth

Figure 2.28 | Vacuum system for synthesis of black phosphorus by short way transport reaction methods.

Figure $2.29 \mid$ The tube furnace and the temperature profile that was used to synthesis black phosphorus.

Figure 2.30 | Pyrex tubes before sealing and evacuated glass (Pyrex) tube after annealing at $600{ }^{\circ} \mathrm{C}$. 
Figure 3.1 | Representative Raman spectra of various samples: (a) $\operatorname{Ag}_{2} \mathrm{O}\left(\mathrm{T}=600^{\circ} \mathrm{C}, \mathrm{P}=\right.$ $10 \mathrm{GPa})$, (b) $\mathrm{Ga}_{2} \mathrm{O}_{3}\left(\mathrm{~T}=600{ }^{\circ} \mathrm{C}, \mathrm{P}=10 \mathrm{GPa}\right)$, (c) Mixed powder of $\mathrm{Ag}_{2} \mathrm{O}+\mathrm{Ga}_{2} \mathrm{O}_{3}(1: 1$, $\left.\mathrm{T}=600{ }^{\circ} \mathrm{C}, \mathrm{P}=10 \mathrm{GPa}\right),(\mathrm{d})$ Mixed powder of $\mathrm{Ag}_{2} \mathrm{O}+\mathrm{Ga}_{2} \mathrm{O}_{3}\left(3: 1, \mathrm{~T}=600{ }^{\circ} \mathrm{C}, \mathrm{P}=\right.$ $10 \mathrm{GPa})$, (e) Mixed powder of $\mathrm{Ag}_{2} \mathrm{O}+\mathrm{Ga}_{2} \mathrm{O}_{3}\left(1: 1, \mathrm{~T}=480{ }^{\circ} \mathrm{C}, \mathrm{P}=10 \mathrm{GPa}\right)$, (f) Mixed powder of $\mathrm{Ag}_{2} \mathrm{O}+\mathrm{Ga}_{2} \mathrm{O}_{3}\left(1: 2, \mathrm{~T}=600{ }^{\circ} \mathrm{C}, \mathrm{P}=\right.$ ambient $)$. Vertical red lines represent the location of our theoretically calculated Raman modes. Vertical light blue lines show the positions of Raman modes calculated by Kumar and Gupta [136]. Vertical dark blue lines show the location of Raman peaks measured by Nagarajan and Tomar [80]. 52 Figure 3.2 Raman spectra of pure silver (I) oxide before (top three curves) and after (bottom curve) DAC experiment. Before applying high pressure, $\mathrm{Ag}_{2} \mathrm{O}$ is unstable under the illumination of $633 \mathrm{~nm}$ laser during Raman measurements. Hence, several shown spectra, measured from the same area at different times differ from each other (top three curves). However, after the high-pressure experiment, $\mathrm{Ag}_{2} \mathrm{O}$ is stable, and its spectrum (bottom curve) does not change under laser illumination. The spectral regions shaded in yellow indicate the location of four peaks characteristic of rhombohedral $\alpha-\mathrm{AgGaO}_{2} \ldots 53$ Figure 3.3 | Gallium (III) oxide before (top curve) and after (bottom curve) applying high pressure. The spectral regions shaded in yellow indicate the location of four peaks characteristic of rhombohedral $\alpha-\mathrm{AgGaO}_{2}$.

Figure 3.4 | Representative Raman spectra of a mixed powder of $\mathrm{Ag}_{2} \mathrm{O}+\mathrm{Ga}_{2} \mathrm{O}_{3}$ with a molar ratio of 1:1 in different pressure and temperature conditions. Characteristic Raman spectrum of rhombohedral $\alpha-\mathrm{AgGaO}_{2}$ is only present in red spectra. 55 
Figure 3.5 | The experimental Raman spectrum of DAC-synthesized $\mathrm{AgGaO}_{2}$ and calculated frequencies of Raman modes for the three known structures of $\mathrm{AgGaO}_{2}$. (There is a five percent uncertainty due to temperature effects.) 56

Figure 3.6 | Electron microscopy characterization of $\mathrm{AgGaO}_{2}$ nanocrystals synthesized during high-temperature DAC experiments: (a) SEM and (b-e) TEM images showing the size and morphology of the nanocrystals. (f) EDX-based elemental mapping was confirming the uniformity and perfect overlap of elemental distributions of $\mathrm{Ag}, \mathrm{Ga}$, and $\mathrm{O}$ (insets)

Figure 3.7 | An EDX spectrum from a typical $\alpha-\mathrm{AgGaO}_{2}$ crystallite synthesized at high pressure. 60 Figure 3.8 | Results of the FullProf-based pattern matching analysis (black curves) of the radial intensity distribution (red curve) of the averaged SAED pattern of the DACsynthesized $\mathrm{AgGaO}_{2}$ for crystal structure models of $\mathrm{AgGaO}_{2}$ with (a) $\mathrm{R} 3 \mathrm{~m}$ (166), (b) P63/mmc (194) and (c) Pna21 (33) space groups. It can be seen that the best fit was obtained for the R3m (166) space group.

Figure 3.9 | (a) A SAED ring pattern acquired from a typical cluster of a few $\mathrm{AgGaO}_{2}$ crystallites. (b) The SAED pattern obtained after averaging the data from about 40 separate clusters. (c) The optimized crystal structure of $\alpha-\mathrm{AgGaO}_{2}$ using our DFT method (see text). (d) Experimental intensity profile obtained after rotational averaging of the pattern shown in $b$ (red curve) and diffraction pattern calculated using the pattern matching mode of the FullProf software suite (black curve) 62 Figure 3.10 | TEM-based single crystal study of individual $\alpha-\mathrm{AgGaO}_{2}$ crystallite: (a) and (b) low-magnification and high-resolution TEM images; (c) Filtered HRTEM image of 
the area marked using yellow frame in b; (d) corresponding [421] zone axis SAED

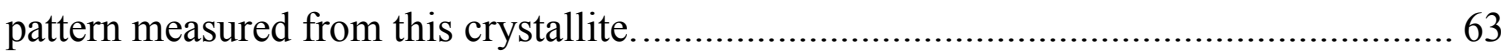

Figure 3.11 | The calculated electronic energy band structure of $\alpha-\mathrm{AgGaO}_{2} \ldots \ldots \ldots \ldots \ldots . . . . . .67$

Figure 3.12 | A SEM Image of 3D Graphene After Etching Ni Foam with HCl 5M (Right) and Photographs of 3D graphene Samples Before and After Etching Ni foam (right).

Figure 3.13 | A representative averages of obtained Raman spectra from 3D graphene foam grown at different temperatures.

Figure 3.14 | Deconvolution of 3D graphene Raman peaks with a Lorentzian function to find FWHM, positions, and area under the peaks for statistical analysis. 71

Figure 3.15 | Raman spectral parameters of synthesized 3D graphene samples at different temperatures. 72

Figure 3.16 | SEM images Show Morphology of a Ni Foam. 73

Figure 3.17 | After the Growth, Graphene Coat Has a Morphology Similar to the Morphology of the Substrate (Ni Foam) 73

Figure 3.18 | Photograph of as-prepared 3D graphene foams synthesized under different growth conditions 74 Figure 3.19 | Scanning Electron Microscopy (SEM) imaging of Ni foam before (top row) and after (bottom row) the graphene growth. 75 Figure 3.20 | a, Transmission Electron Microscopy (TEM) image, b, Selected Area Electron Diffraction (SAED) pattern, and c, Electron Energy Loss Spectroscopy (EELS) of pure $3 \mathrm{D}$ graphene foam prepared by pyrolysis of Citric Acid as the carbon source.... 76 
Figure 3.21 a, Transmission Electron Microscopy (TEM) image, b, Selected Area Electron Diffraction (SAED) pattern, and c, Electron Energy Loss Spectroscopy (EELS) of Nitrogen-doped 3D graphene foam prepared by caffeine as the carbon source. 77 Figure 3.22 | TEM Analysis of Top: Pure 3D Graphene (precursor: Citric Acid) and Bottom: In Situ Nitrogen doped 3D Graphene (precursor: Citric Acid + Caffeine) ....... 78 Figure 3.23 | Raman spectroscopy of nitrogen-doped 3D graphene foam (three upper spectra) and Raman spectrum of pure 3D graphene foam (red) all grown at $1000^{\circ} \mathrm{C} \ldots . .79$ Figure 3.24 | SEM Images of a Nitrogen Doped Sample 79 Figure 3.25 | Deconvoluted high-resolution C (1s) X-ray photoelectron spectroscopy (XPS) spectra of Nitrogen-doped 3D graphene foam using Urea as the carbon source... 80 Figure 3.26 | Deconvoluted high-resolution N (1s) X-ray photoelectron spectroscopy (XPS) spectra of Nitrogen-doped 3D graphene foam prepared by Urea as the carbon source. 81

Figure 3.27 | nitrogen-bonding configurations in the nitrogen-doped carbon 81 Figure 3.28 | Catalytic activity of Nickel foam, Three-dimensional graphene network on nickel foam (Ni-G), Carbon doped with Nitrogen (polyaniline) with core-shell of 3D graphene (Ni-PG), and Silver film (Ag Film) ...................................................... 82

Figure 3.29| Ruby fluorescence spectra at different gas membrane pressures (measured by gas controller unit). These pressures correspond to the actual pressures inside the DAC.

Figure 3.30 | The ruby fluorescence R2 line peak position as a function of pressure inside the gas membrane (measured by gas controller unit). 85 
Figure 3.31 | Pressure inside the diamond anvil cell as a function of gas membrane

pressure. 86

Figure 3.32 | Raman spectra of a few-layer black phosphorus (sample no. 1) under pressure range of $0 \mathrm{GPa}$ (bottom spectrum) to $13.4 \mathrm{GPa}$ (top spectrum) with vertical displacements for clarity. 87

Figure 3.33 | Raman spectra of a few-layer black phosphorus under pressure range of 0 $\mathrm{GPa}$ (bottom spectrum) to $12.56 \mathrm{GPa}$ (top spectrum) with vertical displacements for clarity. 88

Figure 3.34 | Raman shift of three prominent peaks of phosphorene as a function of pressure. 94 Figure 3.35 | Raman $A_{1 g}$ peak position of phosphorene vs. pressure (red) and its linear fit (black) 95

Figure 3.36 | Raman shift of $\mathrm{A}_{2 \mathrm{~g}}$ mode of phosphorene vs. pressure (blue) and its linear fit (black) 95

Figure 3.37 | Raman shift of $\mathrm{B}_{2 \mathrm{~g}}$ mode of phosphorene vs. pressure (green) and its linear fit (black) 96

Figure 3.38 | Raman shift of a full pressure cycle of phosphorene from normal pressure (bottom spectrum) to $13 \mathrm{GPa}$ (indicated by the arrow) and returning to ambient pressure (upper one) 97

Figure 3.39 | Normalized Raman spectra of phosphorene vs. pressure 98 Figure 3.40 | Full-Width Half Maximum (FWHM) of $\mathrm{A}_{1 \mathrm{~g}}$ Raman mode as a function of the pressure. 98 
Figure 3.41 | Full-Width Half Maximum (FWHM) of $\mathrm{A}_{2 \mathrm{~g}}$ Raman mode as a function of the pressure.

Figure 3.42 | Full-Width Half Maximum (FWHM) of $B_{2 g}$ Raman mode as a function of

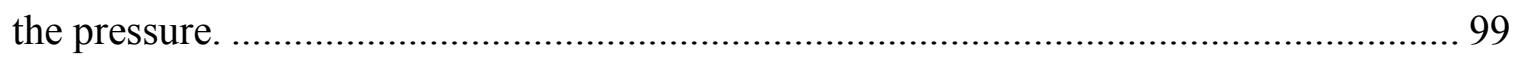




\section{CHAPTER 1: BACKGROUND}

\subsection{High-Pressure Physics and Chemistry}

History of high pressure is about understanding the behavior of matter under extreme conditions and it is extended from planetary science to nuclear explosion and semiconductor physics to crystallography [1]. Pressure is an important thermodynamic variable, which has been less explored comparing to temperature and other thermodynamic variables. High pressure can tremendously influence the physical and chemical properties of materials. Pressure is a valuable tool for synthesizing new phases of material with enhanced properties, and for investigating the available phases. Under extreme conditions interatomic distances, electron configuration, density, and free energy of materials can be modified [2]. Pressure variation range in nature extending over 60 orders of magnitude $[3,4]$ and the range of attainable static pressures with current equipment available in research laboratory exceeding more than 10 orders of magnitude [5]. In this regards pressure is unique among physical variables. In the universe pressure is ranging from the $\sim 10^{-27} \mathrm{~Pa}$ of hydrogen gas in the intergalactic spaces to the $\sim 10^{30} \mathrm{~Pa}$

in center of neutron star and its available domain in laboratory is from $\sim 10^{-12}$ to $\sim 10^{14}$ Pa.[6-9] 


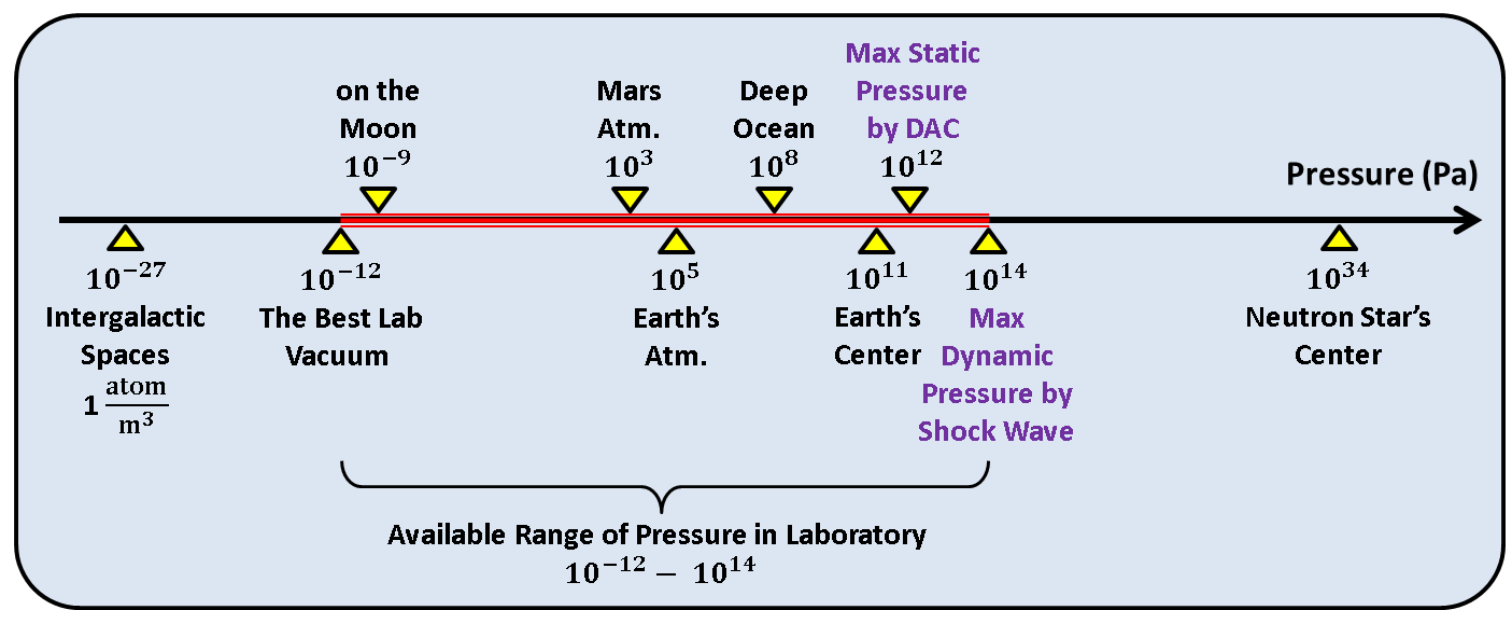

Figure 1.1 | Range of pressure in the universe and obtainable range in available laboratory equipment $[3,4,6-12]$.

Since pressure significantly influences the reaction equilibrium [13] it can be utilized to produce metastable materials. Many thermodynamically metastable materials that synthesized under high pressure can remain kinetically stable at normal pressure for a long time [14]. High pressure can facilitate synthesis of metastable materials by significantly reducing the reaction temperature. High-pressure synthesis of metastable materials are important because many of novel and valuable materials are metastable. Under high pressure, volume changes considerably therefore properties can be explored and tuned with respect to interatomic distances. In contrast to high pressure tuning, chemical modification and modification with temperature have many disadvantages such as imposing melting, boiling, phase separation, disorder, and substantial variations in entropy. The data obtained during high pressure probing and tuning can be used to understand properties of existing materials and designing novel materials for the first time. 


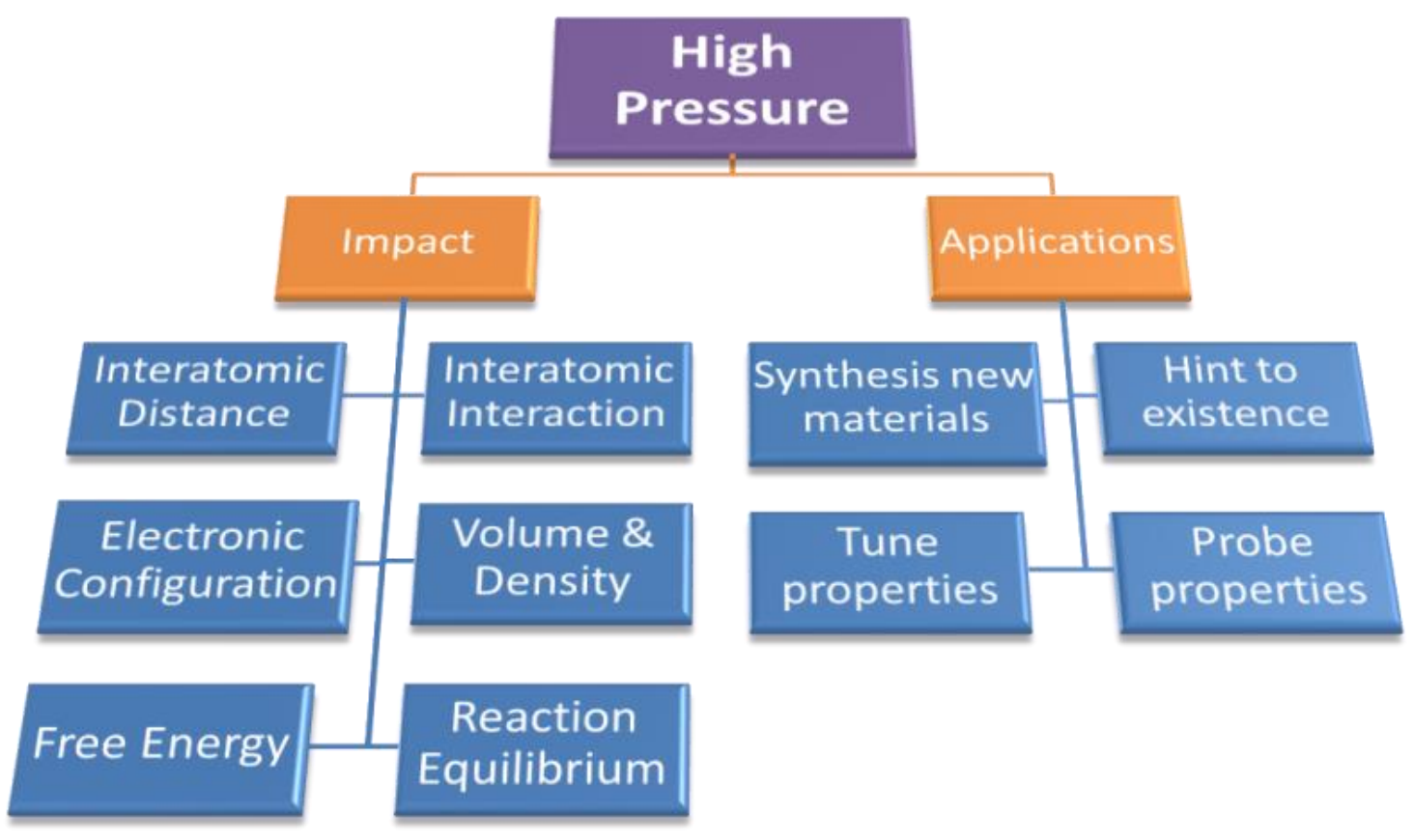

Figure 1.2 | High pressure impact on materials and its applications in materials science.

Fundamental thermodynamic variables (pressure, temperature, and concentration) control the chemical reactions, the equilibrium, and rate of materials transformations. However, in solid state of materials, only temperature and concentration influence on the chemical reaction [15] and equilibrium have been investigated thoroughly and the effect of high pressure is remained less explored. One of the reasons behind the underutilizing the high pressure in materials science in comparison with other thermodynamics parameters is the fact that the equipment of high pressure experiment is not routinely available in physics and chemistry laboratory and they are limited to specialized laboratory. The other reason is the very small volume of the samples in high pressure experiment as it is not possible to reach very high pressure in large volume. Recently, the number of high pressure research works has growth explosively and the study of materials under extreme conditions has attracted substantial attentions among scientist [16]. The main reason for 
this rapid increase is the latest advancement in both experimental and theoretical capabilities that have simplified the high-pressure experiments.

The small volume of the sample and its chamber opening in high-pressure experiments make the signal to noise ratio considerably small and consequently severely hampered an effective in situ measurement. However, development in high pressure instrumentation help researcher to overcome the limitations that arise from the sample size [17]. In last few years, advances in high pressure equipment specially improvement in design of diamond anvil cell (DAC) has led to major breakthrough in application of high pressure in materials science [1]. Nowadays, using a diamond Anvil Cell (DAC), one can achieve to the pressure over $700 \mathrm{GPa}[11]$ and also using laser heating techniques to the temperature up to $\sim 6000{ }^{\circ} \mathrm{C}[18]$. Recent developments in high-pressure equipment have created new opportunities for investigating the behavior of materials at high-pressures. Diamond anvils in DAC is transparent to wide range of electromagnetic radiation and this property of diamond along with its hardness make diamond anvil cell (DAC) extremely powerful tool for various in situ high-pressure measurements. Currently, using DAC a broad range of probing techniques can be performed from X-ray and neutron diffraction to spectroscopic techniques such as Raman, Fourier transform infrared (FTIR) and photoluminescence (PL).

Advances in theoretical and computational calculations also antributed to the rapid growth in high pressure research works. On the one hand, developments in calculation algorithm $[19,20]$ and on the other hand sharp increases of computers power resulted in reliable theoretical predictions of phase stability [17] and also calculation of structural, vibrational and electronic properties of materials accurately. This theoretical data can 
provide research direction for high pressure experiments and also help to interpret the experimental results $[1,21]$.

The most frequent computational modelling method in high pressure research is density functional theory (DFT) $[22,23]$ with local-density approximations (LDA) to the exchange-correlation (XC) energy functional [24-27]. In first principles theoretical calculation approaches like DFT pressure can be concidered as a probe to study variations in properties of condens matter as a function of interatomic distances. Therefore influence of pressure compare to temperature can be incorporated into ab initio modelling easier which need sampling of several configurations. This makes pressure very attractive thermodynamic parameter for first principles calculations [1]. High pressure and its sever impact on iteratomic ditances and consequently overlap of the electron clouds increase the energy of the electrons and put the system in very unstable state. Therefore, the system try to recover and minimized the free energy which leads to several phenomena, namely, amorphization, condensation, polymerization, direct to indirect bad gap transition, dissociation, ionization, semiconductors to superconductors transition, ferromagnetic to paramagnetic transition, crystals phase transformation, and metallization $[28,29]$. 


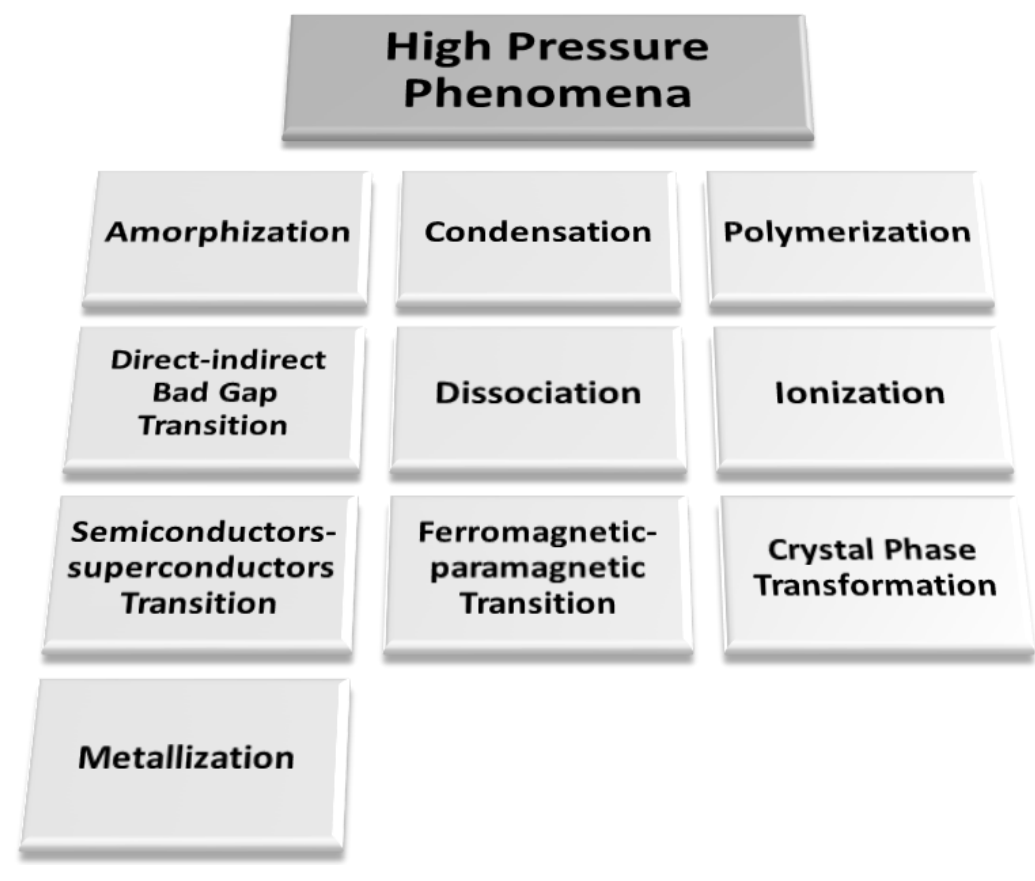

Figure 1.3 Phonomena under High Pressure [30].

\subsection{The Nobel Prizes for High-Pressure Research}

Three nobel prizes in physics and chemistry for high pressure related research are another indication of the impotance of high pressure. In 1918, a German chemist, Fritz Haber (1868 - 1934), was received the Nobel Prize for invention of a high pressure method to synthesize ammonia [31]. In 1931, Carl Bosch (1874 - 1940) and Friedrich Karl Rudolf Bergius (1884 -1949), who were both German chemists, were received the Nobel Prize for the invention and development of chemical high-pressure methods [32]. In 1946, Percy Williams Bridgman (1882 -1961), an American physicist won the physics Nobel Prize for his high pressure research [33].

\subsection{Kinetics, Thermodynamics and Metastability}

In any chemical reaction or phase transformation one can think of atleast three enegy state for the system during the process as it shown in Figure 1.4. 


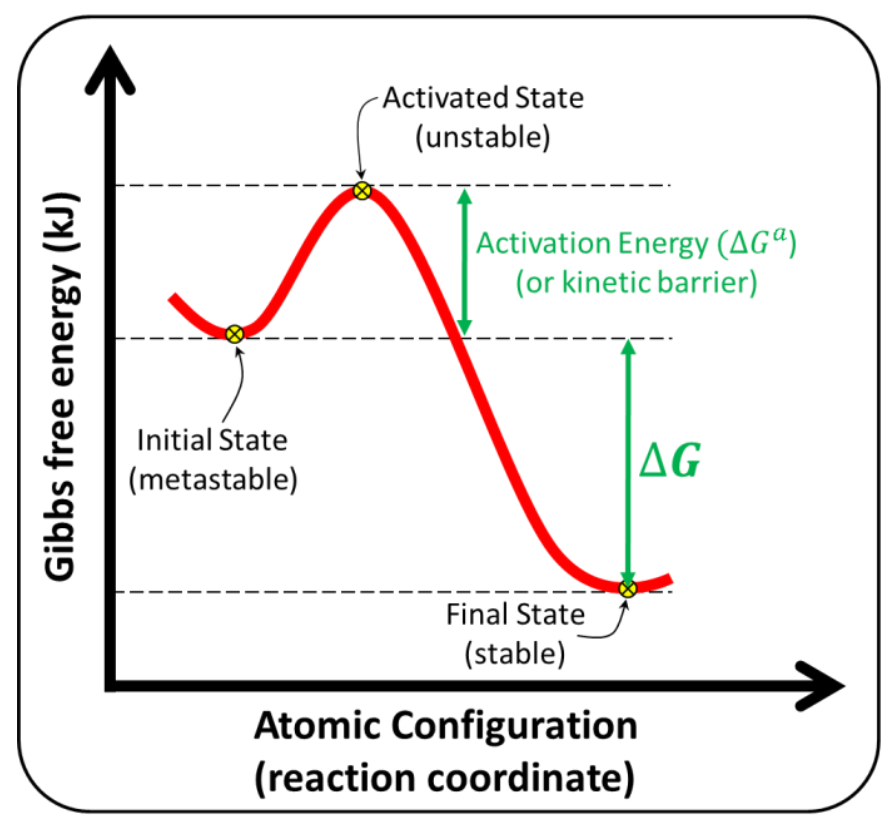

Figure 1.4 | Energy Profile Diagram of a Phase Transformation.

Activation energy (kinetic barriers) determines the transformation rate of a process from initial state to the final [34] equlibrium. When the rate of transformation is very slow due to the kinetic barrier and there is an alternative phase (metastable) with lower energy barrier the crystal may transform to the metastable phase instead of the stable one [14]. Many metastable phases have such a long lifetime that experimentally they are not distigushable with equlibrium phases. Interstingly, metastable phases are common amoung materials with covalent bonds therefore many semiconductor structures are metastable. Diamond is an example of metastable structure with long liftime that if heated at high temperature transforms to more stable graphite phase.

\subsection{Semiconductors under High Pressure}

Semiconductors are significantly important materials with extremely wide range of applications from electronic and information technology to energy harvesting and energy 
storage[17]. Majority of semiconductors are covalently bonded materials and consequently the metastable phases are very common among semiconductors. Furthermore, semiconductor materials naturally tend to transform progressively to a denser structures through a chain of high pressure structural transitions. As a result of the metastablility and typical tendency toward high-pressure phase transitions, studies of semiconductors under high pressure become considerably important. High pressure can severly affect the interatomic distances and consequently all the interatomic interactions which control materials properties. In other words high pressure impose such a drastic changes to semiconductor physical properties that is not possible to achieve by temperature variation alone. Band gap closure and metalization or indirect to direct band gap transition are exaples of high pressure influence on semiconductors. Typically, electron bands get broader under high pressure and this leads to a band gap reduction. Moreover, band gap closure and metallization commonly occur in the first step of phase transitions under high pressure.

Another important advantage of high pressure probing of semiconductors is the possibility of studying the defect nature in these materials. This is possible because the high pressure can locally change neighboring defect structure and move its correlated electronic states. However, the amount of pressure needed to change the local structure around a crystal defects is relatively low compare to pressure needed for phase transitions.

\subsection{Conditions for Stable Phases}

In a structure transition process Gibbs free energy $(G)$ of the system determines the stability of the final phase. In other words during the tarnsition process Gibbs free enrgy 
of the system must be minimized to reach the equlibrium phase at specific temperature and pressure. For Gibbs free energy we have:

$$
G(P, T)=U+P V-T S
$$

where $U$ is the internal energy (joul), $P$ is pressure (pascal), $V$ is the volume $\left(\mathrm{m}^{3}\right), T$ is the temperature (kelvin) and $S$ is the entropy (joule per kelvin).

In a high pressure experiments we are dealing with a system that $\mathrm{P}$ and $\mathrm{T}$ are external parameters, and other variables in Gibbs free energy function (i.e. $\mathrm{U}, \mathrm{V}$, and $\mathrm{S}$ ) are free to adjust in a way to minimize Gibbs free energy. That is to say we work with statistical constant pressure ensemble.

In equation (1) it is evident that by increasing the pressure, phases with smaller volume are more favorable. Furthermore, temperature and entropy are linked together according to the equation and we know configurational entropy are same for all solids crystals. This makes temperature less effective in solids structural transformation compare to pressure. In high pressure phase transformation process if the pressure is hydrostatic there is another condition that must be satisfied for the stable structures. That is the stress tensor $\sigma_{i j}$ must be in diogonal form:

$$
\sigma i j=\frac{d^{2} U}{d \varepsilon_{i} d \varepsilon_{j}}=\left(\begin{array}{ccc}
P & 0 & 0 \\
0 & P & 0 \\
0 & 0 & P
\end{array}\right)
$$

In this equation $\varepsilon_{i}$ and $\mathrm{P}$ are strain in $i$ direction external pressure, respectively. If above two conditions are satisfied for a specific crystal structure then it can be concidered as the stable phase after the transformation process [1]. 
Table 1.1 | In-situ High Pressure Probes with references.

\begin{tabular}{|c|c|c|c|}
\hline \multicolumn{4}{|c|}{ In Situ High Pressure Measurements } \\
\hline X-ray Diffraction (XRD) & {$[35-38]$} & UV - Vis Spectroscopy & [39] \\
\hline Single-Crystal X-Ray Diffraction & {$[38,40,41]$} & Resistivity Measurement & [42-44] \\
\hline X-ray Absorption Spectroscopy (XAS) & {$[38,45,46]$} & Raman Spectroscopy & {$[47-50]$} \\
\hline $\begin{array}{l}\text { Extended X-ray Absorption Fine } \\
\text { Structure (EXAFS) }\end{array}$ & {$[34]$} & Neutron Diffraction & {$[51]$} \\
\hline $\begin{array}{l}\text { X-ray Absorption Near Edge Structure } \\
\text { (XANES) }\end{array}$ & {$[45]$} & Mössbauer Spectroscopy & {$[52,53]$} \\
\hline X-ray Fluorescence (XRF) & {$[45]$} & Impedance Measurements & {$[54]$} \\
\hline $\begin{array}{l}\text { X-ray Magnetic Circular Dichroism } \\
\text { (XMCD) }\end{array}$ & {$[46]$} & Hall Effect Measurement & {$[55]$} \\
\hline Hall Effect Measurement & {$[55]$} & Infrared Spectroscopy & {$[38,48,50]$} \\
\hline Brillouin Spectroscopy & {$[56,57]$} & FTIR Spectroscopy & {$[48,58]$} \\
\hline Photoluminescence Spectroscopy & {$[59,60]$} & $\begin{array}{l}\text { X-ray Raman } \\
\text { Spectroscopy }\end{array}$ & [61] \\
\hline $\begin{array}{l}\text { Nuclear Resonant Inelastic X-Ray } \\
\text { Scattering }\end{array}$ & {$[61,62]$} & Ultrasonic Interferometry & {$[63,64]$} \\
\hline $\begin{array}{l}\text { Resonant Inelastic X-Ray Scattering } \\
\text { Spectroscopy }\end{array}$ & {$[61]$} & Optical Interferometry & {$[65]$} \\
\hline $\begin{array}{l}\text { Electronic Inelastic X-Ray Scattering } \\
\text { Spectroscopy }\end{array}$ & {$[61]$} & & \\
\hline
\end{tabular}

\subsection{Rhombohedral $\alpha-\mathrm{AgGaO}_{2}$ (Semiconductor with $\mathrm{ABC}_{2}$ Form)}

As mentioned earlier, in materials science and solid-state physic, high-pressure techniques are employed for tuning a variety of material properties such as magnetic ordering, electronic and heat transport and optical response. Moreover, they are being used in synthesizing non-equilibrium phases or novel compounds [2,66-68]. Applying 
high-pressure leads to shrinkage of the inter-atomic distances and enhancing the reactivity of materials [30].

In particular, high-pressure techniques are useful in cases of decomposition of one of the precursors under normal pressure (i.e., at 1 atm.) and at temperatures below the reaction temperatures. Applying high-pressure increases the decomposition temperature and consequently, stabilizes the precursor. This method has been reported for stabilizing $\mathrm{HgO}[69], \mathrm{MnO}_{2}$ [70] and $\mathrm{PbO}_{2}$ [71].

In this work, we synthesized $\alpha-\mathrm{AgGaO}_{2}$ [72] by mixing $\mathrm{Ag}_{2} \mathrm{O}$ and $\mathrm{Ga}_{2} \mathrm{O}_{3}$ powders under high-pressure. Since $\mathrm{Ag}_{2} \mathrm{O}$ is instable under the atmospheric pressure and undergoes decomposition at a temperature of about $300^{\circ} \mathrm{C}[73-76]$, it is challenging to synthesize $\mathrm{AgGaO}_{2}$ in a single step using the common open-system methods. Thus, producing silver delafossite has been done using closed-system techniques such as cation exchange (metathetical) [77], hydrothermal reactions [78], oxidizing flux [79] and ultrasound assisted reactions [80]. However, each of these methods imposes different limitations such as producing undesired by-products. Also, they are time consuming and require multiple steps $[79,81,82]$.

\subsection{Three-Dimensional (3D) Graphene Architecture}

For years, graphene materials family has been studied intensively and remains in the center of researchers' attention in the field of materials science owing to its astonishing properties. Combinations of the tremendously large surface area, elegant electrical and thermal conductivity, superior chemical stability, and extreme mechanical strength make graphene an essential component for fabricating functional materials for a broad range of applications. However, practical limitations such as the difficulty of mass 
production of high-quality samples render a barrier to extensive implementation of graphene in practical devices. For example, during the processing of graphene into an actual device, the strong $\pi-\pi$ interaction between graphene sheets cause the isolated graphene flakes to restack to form graphite-like powders which can substantially reduce the specific surface area and thereby resulting in inefficient utilization of the graphene layers [83-89].

Recently, three-dimensional structures of graphene have attracted tremendous attentions as an effective way to utilize the distinctive inherent properties of graphene sheets in practical applications. Three-dimensional graphene-based materials offer an easy and versatile platform for functionalization and integration into devices. Furthermore, the interlocking of graphene sheets into 3D structures solve the restacking issue and make 3D graphene-based materials more compatible with conventional material processing. As a result, 3D graphene materials and their derivatives triggered extensive studies for numerous potential applications in energy, electrochemistry, bioelectrochemistry and pollution management [90-97].

Particularly, in the field of energy, the superior performance of both electric double-layer capacitors and pseudocapacitors can be achieved by functionalization of 3D graphene foam due to its unique structural properties. 3D Graphene foam structure enhances the access of electrolyte with its surfaces while providing electrically conductive pathways for the functional materials. Generally, in electrode systems, the porous structures of 3D graphene facilitate incorporation of active materials to form 3D graphene-based composites. Therefore, the performance of the electrode systems is enhanced by the combination of 3D graphene structural features and electrochemical 
activity of incorporated materials. Moreover, 3D graphene-based materials have been identified as an ideal candidate for environmental applications. The exceptional large surface area along with well-defined porous arrangement allow 3D structures of graphene to capture molecules of contaminations efficiently [98-101]. Also, three-dimensional graphene-based materials can be recycled easily. Removal of oils, organic dyes and solvents, heavy metals, and gas pollutants are few examples of environmental applications for 3D graphene-based materials [102-104].

\subsection{Few-Layer Black Phosphorus (Phosphorene)}

Since 2004 that graphene was discovered [105], there has been a search for new twodimensional (2D) materials. Phosphorene is one of the newly discovered 2D materials with most interesting properties and multiple possible applications [106]. A single atomic layer of black phosphorus (BP) is called phosphorene. Two-dimensional materials are composed of a single-atom-thick layer and can be categorized into diverse groups [107$110]$.

Despite the superior properties of Graphene such as high carrier mobility, ultrahigh surface area, excellent thermal conductivity, and quantum confinement effect [111] the lack of band gap limits its electronic applications. However, BP has a highly tunable thickness-dependent band gap. It can be changed with functionalization, doping and the number of layers from $\sim 1.5 \mathrm{eV}$ (monolayer) to $0.3 \mathrm{eV}$ (bulk BP) [112]. Other significant properties of BP include high carrier mobility, and strong in-plane anisotropy, particularly the anisotropy of electric conductance [113]. Moreover, the carrier mobility and on/off ratio are layer-dependent as well [114]. 
BP was first discovered by Bridgman more than a century ago [115]. Similar to graphite, it is a van der Waals material, and is comprised of vertically stacked 2D sheets. Unlike graphite, the in-plane bonding in BP stems from $\mathrm{sp}^{3}$ hybridization which prevents phosphorene from forming atomically flat sheets [116]. They arrange a puckered honeycomb-structured layers [117]. The layers are attached together by weak van der Waals forces[118]. The electronic band gap of bulk BP is $0.3 \mathrm{eV}$, which is much smaller than the predicted $1.5 \mathrm{eV}$ value of phosphorene. 


\section{CHAPTER 2: EXPERIMENTAL METHODS}

\subsection{High-Pressure Equipment}

Diamond anvil cell (DAC) is a small device to generate extreme thermodynamic conditions i.e. high pressure and high temperature. DAC consists of two opposing diamond anvil with small flat culet. DAC compresses small samples between diamond culets and can generate very high pressure of above $100 \mathrm{GPa}$ easily [11]. The current record of pressure with DAC is about $1 \mathrm{TPa}$ [119]. Moreover, samples in DAC can reach to the temperature as high as $5000 \mathrm{C}$. The first diamond anvil cell was made by Charlie Weir in 1958 in the NBS laboratory[120].

\subsection{Diamond Anvil Cell (DAC)}

Diamond anvil cell (DAC) was developed by Weir et al. and Van Valkenberg [120] for generating high-pressure conditions. This device followed the pioneering work in high pressure by Percy W. Bridgman [33]. They replaced the tungsten carbide in traditional anvils with a diamond, which is significantly smaller and harder. Moreover, the optical properties of diamond allow in-situ spectroscopy at high-pressure conditions.

The DAC is composed of two opposed diamond anvils (Figure 2.1). Due to the smaller surface area at the tip of the diamond, the pressure is considerably higher in this region comparing to the back of the diamond (reffered as table). A metallic gasket is placed between the flattened tips (called culet) to prevent them from touching. The gasket is drilled at the center to create a sample chamber where a pressure transmiting medium 
surrounds the sample. Applying a small force to the diamond tables will apply a significant pressure to the sample. The pressure is measured using a small amount of ruby between the culets. Using DAC, one can achieve pressures in the gigapascal range $[11,119]$.

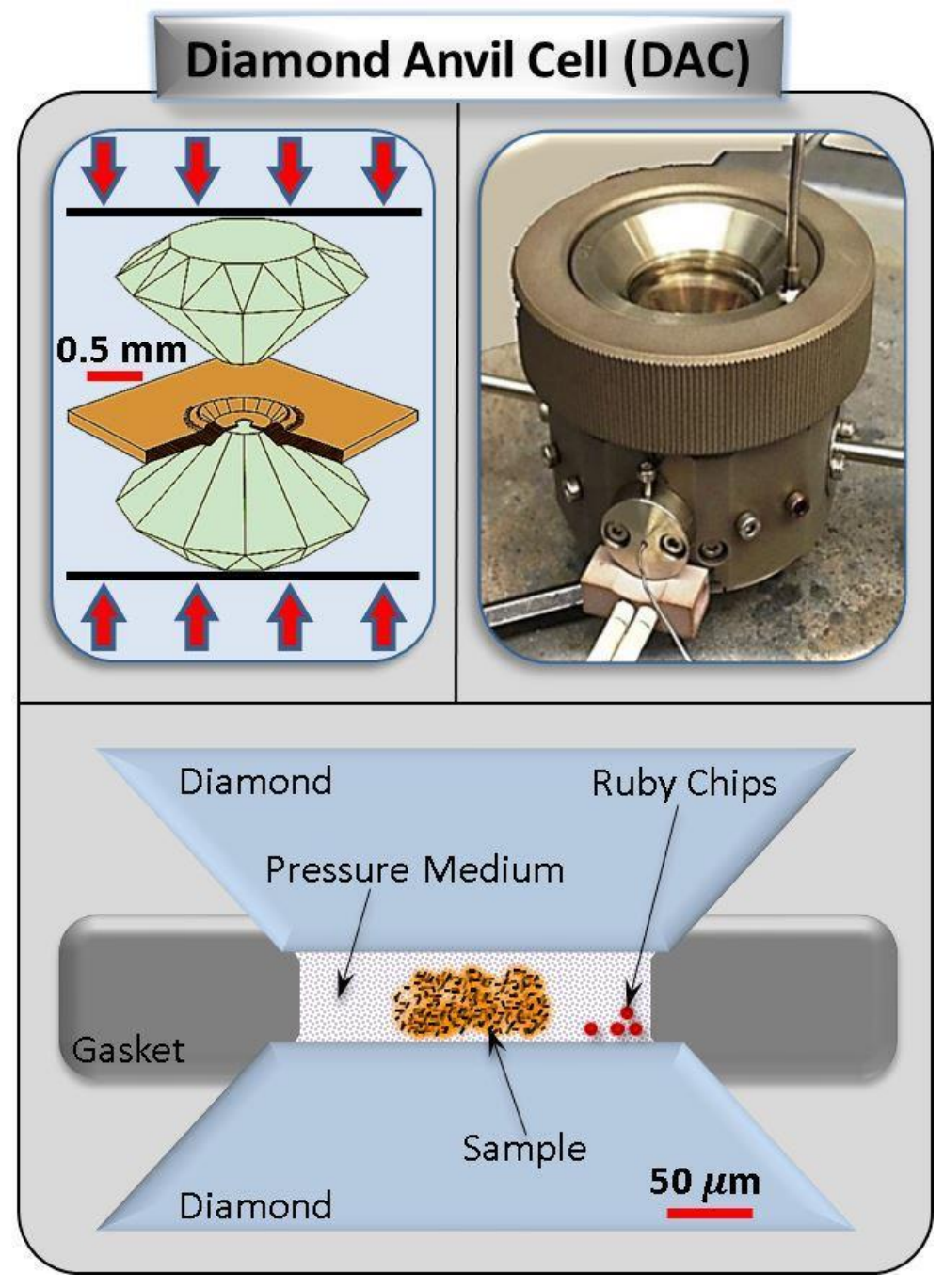

Figure 2.1 | Schematics of the diamond anvil cell (DAC) and a photograph of our gas membrane driven DAC. Typical DAC is composed of two opposed diamond anvils with a metallic gasket in between providing a small chamber for the sample.

The diamonds are attached to the backing plates of the DAC. The pressure can be increased by applying a force on the membrane or by twisting the screws so that the two 
parts of DAC approach further. Since there are four screws, one must make sure to turn all the screws in the same manner. This can be done by keeping the screws at equal height. A caliper can be used to measure the height. It is more reliable to raise the pressure by applying a force on the membrane as the force transforms uniaxially through the membrane.

\subsubsection{Diamond Anvils}

The geometry, purity, and color of the diamond play a deterministic role in the outcome of high-pressure experiments. Hence, it is important to choose the right diamond. This can be done by using the classification of the '4cs' of diamond: carat, clarity, cut and color [121]. A carat is a unit of mass, equal to $200 \mathrm{mg}$ for measuring gemstones and pearls.

The clarity represents the quality of diamond in the sense of impurities. The level of clarity is determined based on the appearance of the diamond under $10 \mathrm{X}$ magnification. The surface and internal defects are called blemishes and inclusions respectively. Inclusions can lead to cracks under high pressures. Moreover, for spectroscopic applications, high clarity diamonds are desired as the defects can shift optical paths causing a drift in the experimental results. A diamond of grade $\mathrm{F}$ (Flawless) shows no blemishes or inclusions under 10X magnification and is the most suitable choice for spectroscopic measurements. 


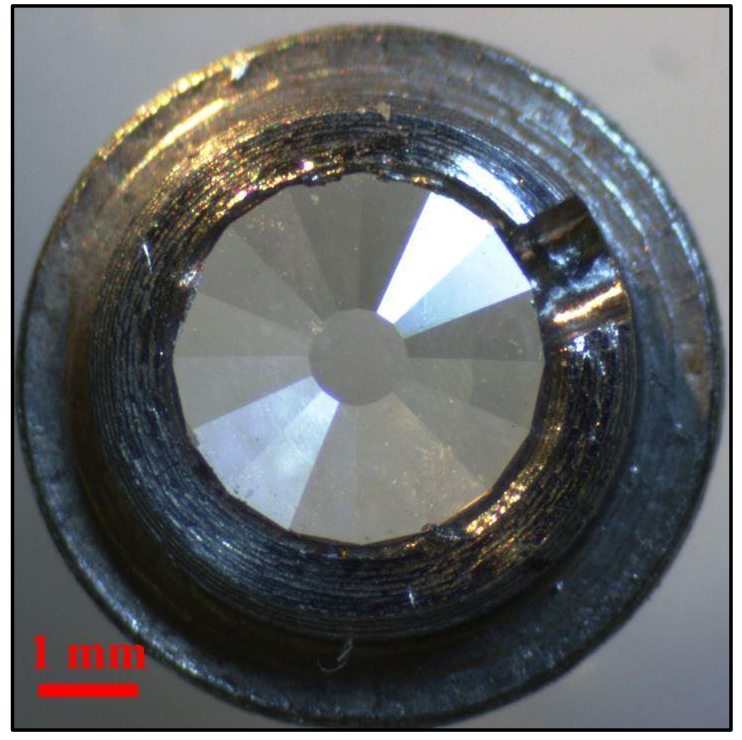

Figure 2.2 | Magnified Photograph of a Diamond Anvil under Optical Microscope.

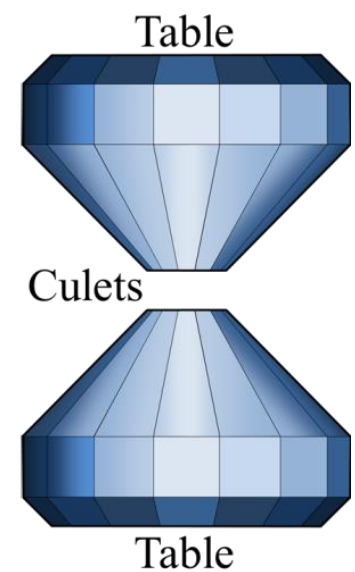

Figure 2.3 | Schematic of two diamond anvils with the standard design cut (Culet and Table of the Anvils is indicated.

The cut and design of the diamond anvil plays a major role in the high-pressure experiments, as the size of the diamond culet is the major factor to find out the maximum attainable pressure. A well-known design is the modified brilliant cut with a smaller distance between the table and the gridle (Figure 2.4). This allows a larger table resulting to a larger area for applying the force [121]. 
Another typical design of diamond anvil is the Boehler-Almax cut (Figure 2.4). BoehlerAlmax cut provides a larger aperture for in-situ X-ray diffraction experiments.

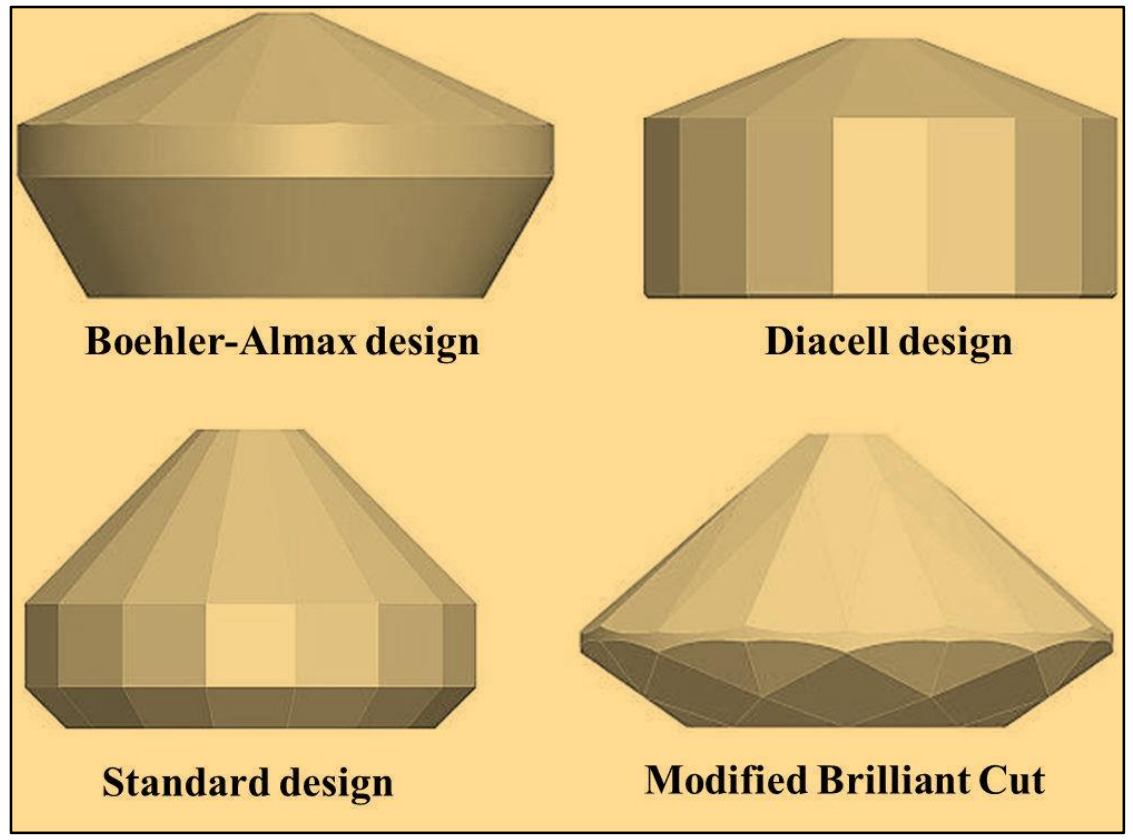

Figure 2.4 | Few Different Diamond Anvil Designs and Cuts that is commercially available from Almax easyLab Inc).

The diamond color scale is set by Gemological Institute of America (GIA). The color of diamond is defined by GIA as the lack of color. They provide a $\mathrm{D}$ to $\mathrm{Z}$ color-grading scale based on the degree of colorlessness where $\mathrm{D}$ and $\mathrm{Z}$ represent "completely colorless" and "pale yellow/brown" respectively. The diamond color is specifically important in the spectroscopic measurements as the fluorescence from the diamond can raise the background signal. Raman spectroscopy can be used to examine the fluorescence level of the diamond.

The DAC is composed of several parts including but not limited to diamond anvils, metal gasket, and backing plates. A crucial step in DAC experiment is putting all the 
components together. For instance, it is important to ensure that diamond anvils are well aligned before loading the sample or applying force to the DAC.

\subsubsection{Backing Plates}

Typically, backing plates (diamond anvil seats) are made of tungsten carbide and hold the diamond anvils in place. Depending on the experiment temperature, different materials can be used to glue the diamond anvils onto the backing plates. Hysol 9437 and STYCAST epoxies are used for high- and low-temperature experiments respectively. Before attaching, the anvils and backing plates must be cleaned carefully as the dust can initiate an asymmetry and consequently a crack in the diamonds at high pressures. A mounting device can be used to hold the anvils and plates in the right position close to each other while applying the glue.

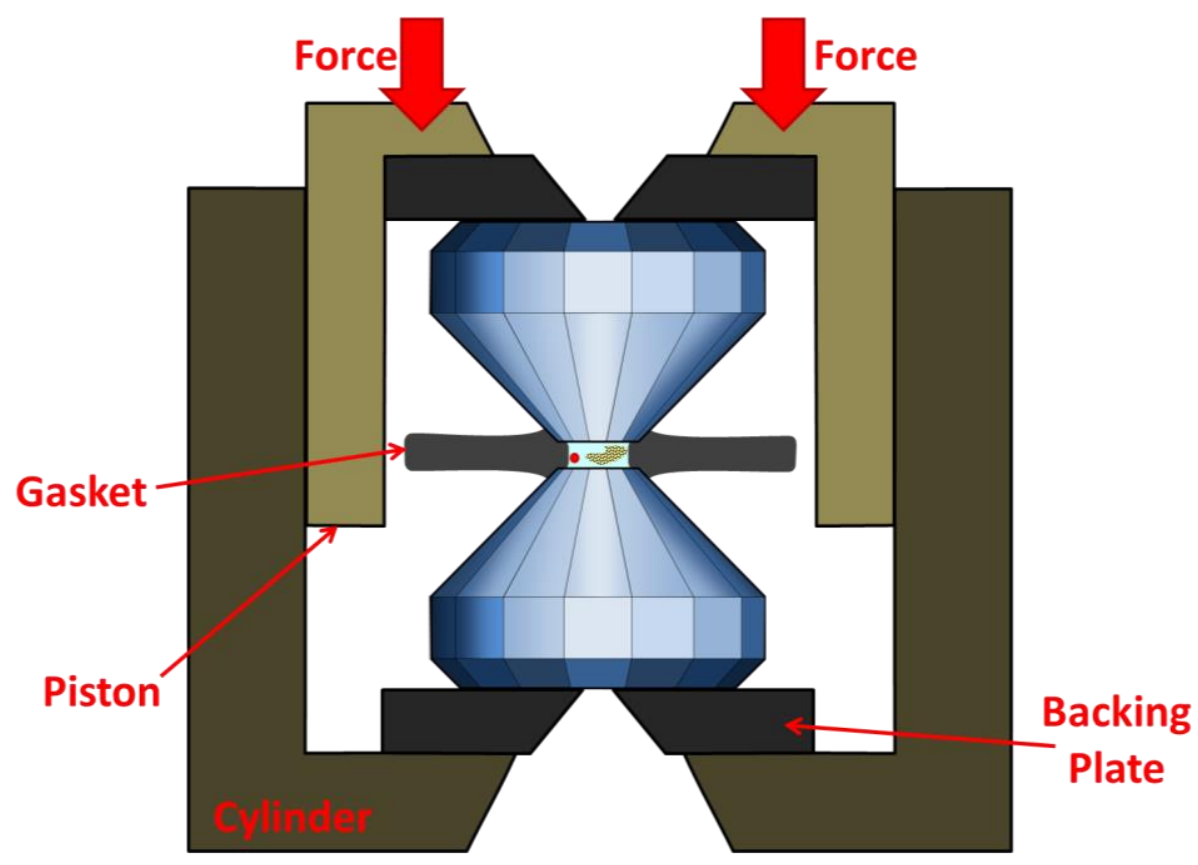

Figure 2.5 | Increasing the pressure inside the sample chamber by applying a force onto the pistons. 
2.2.3 The Alignment of the diamond Anvils and preparing the sample chamber

Other than aligning the anvils with backing plates, the two diamonds must be lined up so that their culets are parallel with each other.

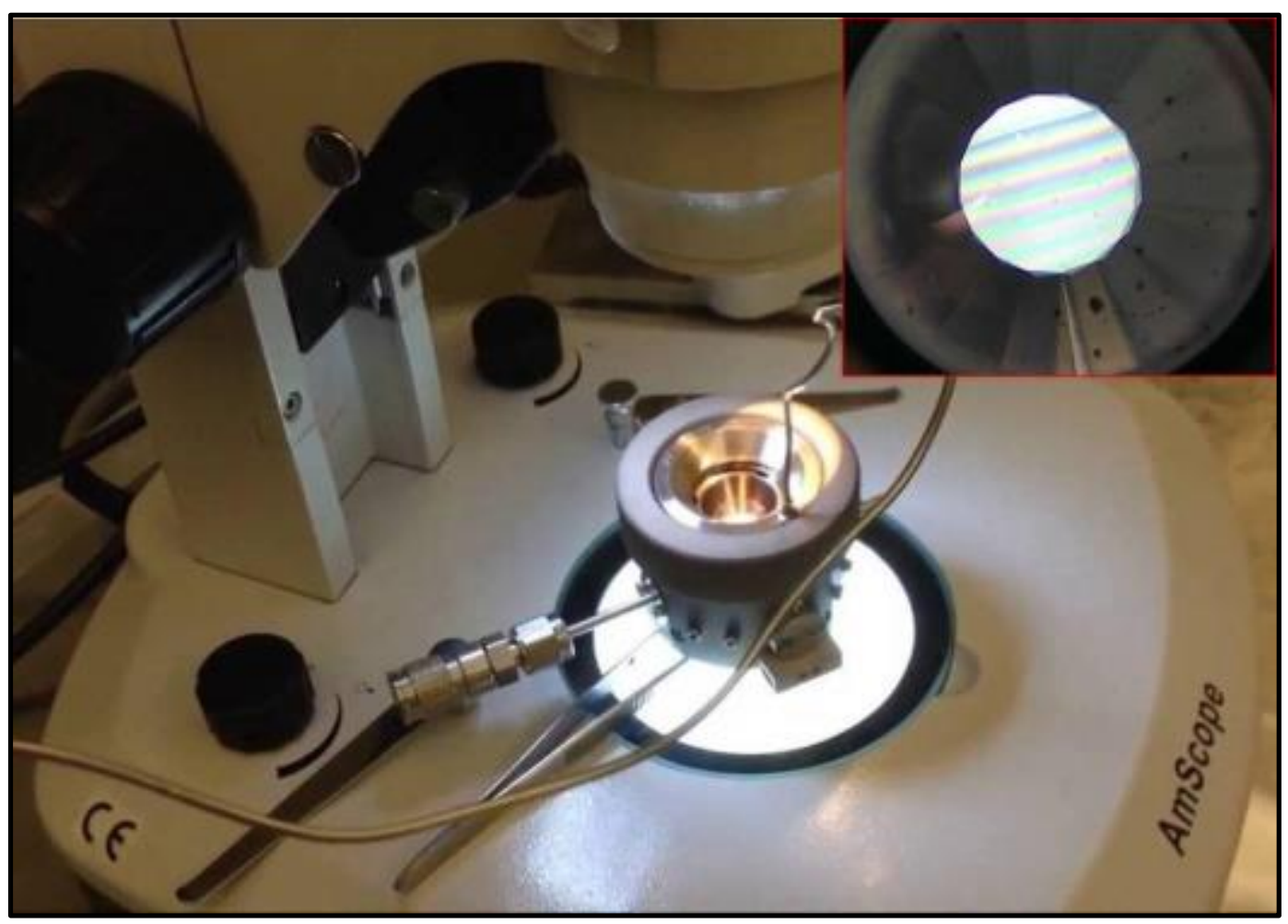

Figure 2.6 | Top View through Diamonds with Optical Microscope Showing the Misalignment of Anvils.

The alignement process should be done under optical microscop. Top and lateral view of diamond anvils when they are close reveal any misalignment. Figures 2.6 and 2.7 show the top and lateral views of anvils under the microscope. In top view (Figure 2.6) under the microscope if the anvils are not parallel the newton rings reveals the misalignment. Moreover, with high magnification optical microscope it is necessary to check all lateral direction to make sure that diamond anvils are aligned perfectly. Any small asymmetry in the system under very high-pressure can resulted in breaking the diamond anvils. 


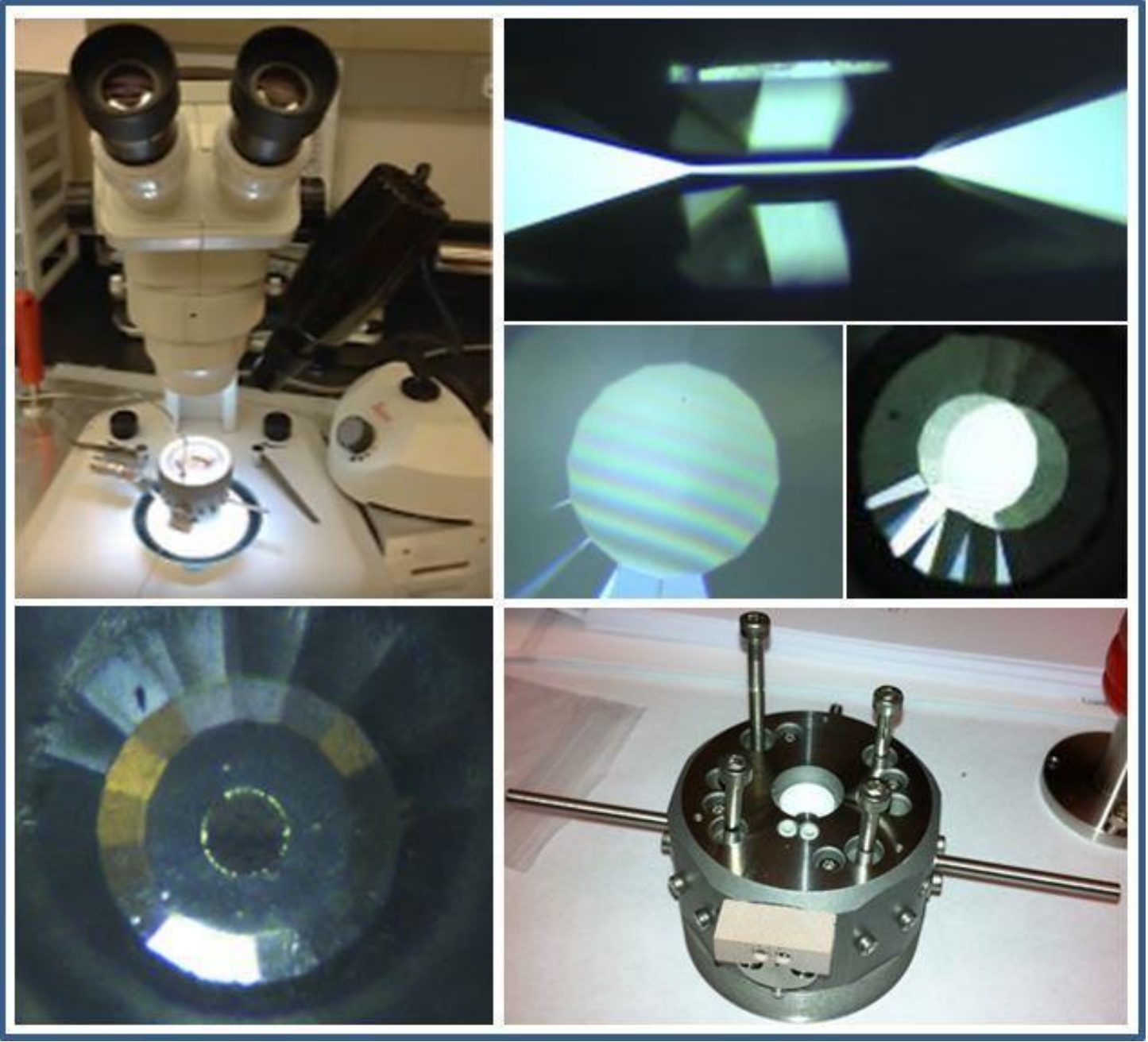

Figure 2.7 | Alignment of the diamond anvils and Loading Samples (Powder) in Gasket Hole (Sample Chamber) under the Microscope

A metallic gasket with a hole at its center is used as a sample chamber between the anvils. Before drilling the hole, the gasket must be preindented between the diamond anvils inside the DAC. Then the hole is drilled at the center of the indentation. The size of the indentation depends on the experiment and the range of desire pressure. A larger one is suitable to achieve higher pressures, and a thinner one is preferred to reach higher temperatures. 


\subsubsection{Drilling Small Hole at Center of the Metallic Gasket}

To create symmetric holes at the center of the gasket, which served as the sample chamber a Micro Electric Discharge Machining (EDM) system from Hylozoic Products was utilized. Since the holes sould be in the range of $\sim 200 \mu \mathrm{m}$, the drilling should be done under the microscope. In this work, we used inconel or stainless steel gasket for the high-pressure experiments. The EDM system was used in combination with an optical zoom microscope (Figure 2.9) to find the center of the indentation accurately. EDM uses electrical discharges to melt the gasket to make the hole.

\section{Diamond}

\section{Gasket}

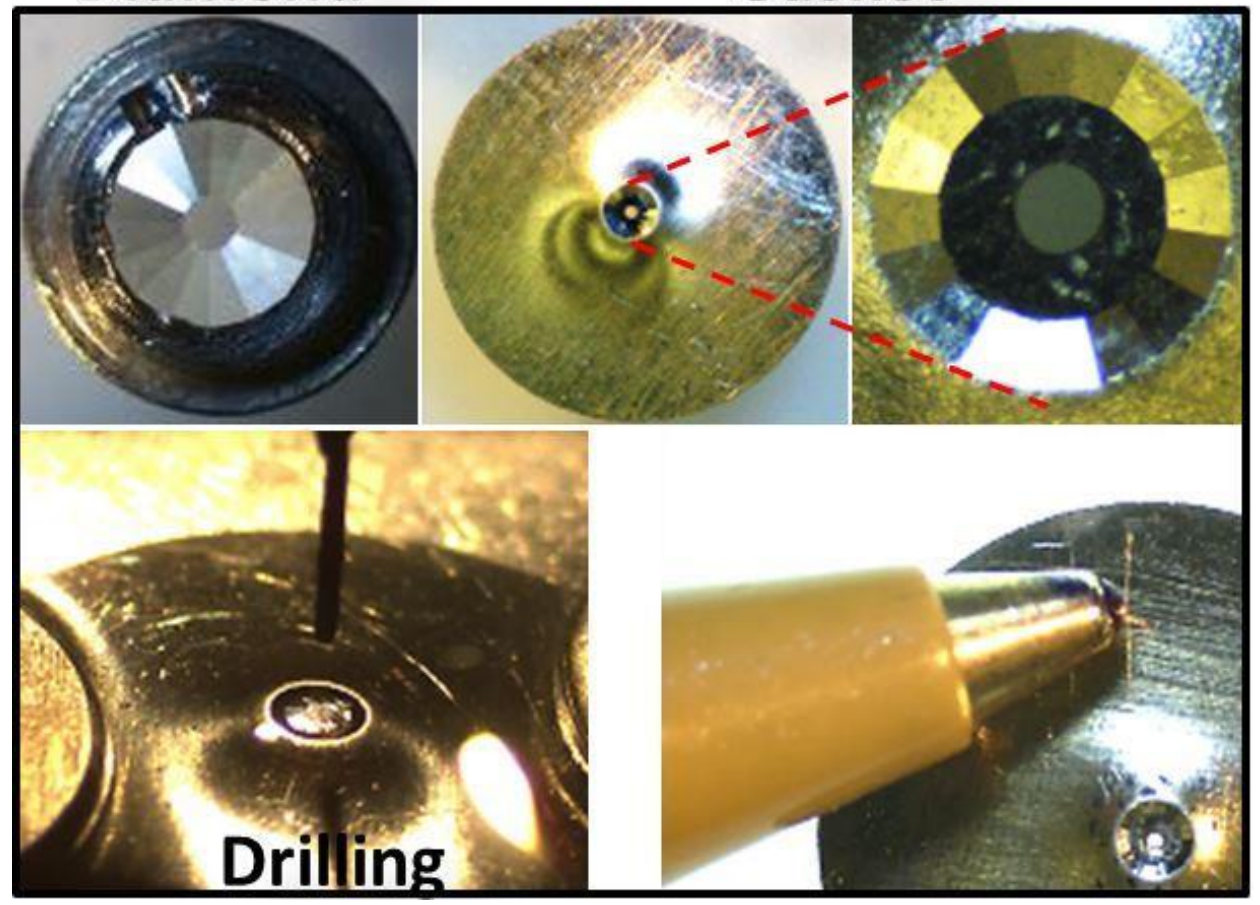

Figure 2.8| Small Hole at the Center of the Metallic Gasket as a Sample Chamber. 


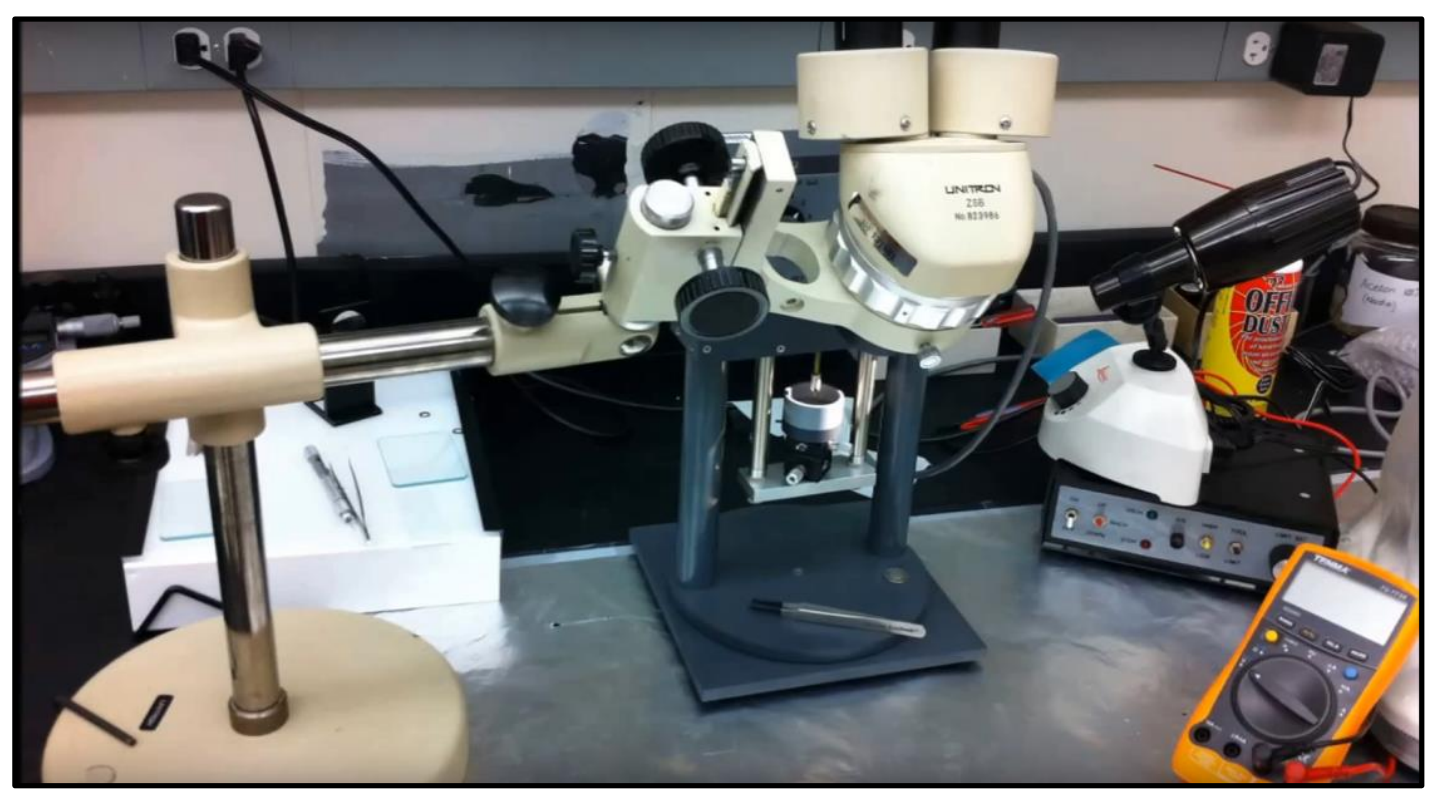

Figure 2.9 | The Micro EDM System and Optical Zoom Microscope.

\subsubsection{Loading of the Sample into the Gasket Hole}

At this stage, the sample chamber is ready to be loaded. The sample should fill about a half of the gasket hole, and it should not be in touch with the gasket if hydrostaticity is important. A typical volume range of the sample is about 50 to $100 \mu \mathrm{m}^{3}$. In the case of powdered samples, further grounding is done using diamond anvils or tungsten carbide cubes. Then the sample can be transferred to the chamber using a needle tip in $\mu m$ range. This process can be done inside a glovebox to avoid sample contamination [121].

\subsubsection{Pressure Transmitting Medium}

The sample must be surrounded with a pressure-transmitting medium (PTM) for hydrostatic pressure. A suitable PTM should have a simple crystal structure. Moreover, it must be chemically inert, highly compressible and very soft. Noble gasses like helium, neon, and argon are among the mediums that can be used as a PTM. Choice of the right 
PTM depends on the experiment parameters. For instance, Helium (He) is employed in studies of the equation of states and Alkali halides like sodium chloride $(\mathrm{NaCl})$ and potassium bromide (KBr) are mostly applied to experiments that involve melting as they feature a high melting point. It is extremely important for the PTM to have a high hydrostatic limit. Otherwise, it can be contributed to the phase transition of the sample, which is not desiredand may cause wrong conclusions. It is more straightforward to use a liquid or solid PTM rather that a gaseous one as using gas as the PTM is involved difficult gas loading procedure [122-125].

\subsubsection{Heating Techniques}

There are several methods for increasing the temperature inside a DAC, laser heating, internal resistive heating and using external furnace are typical DAC heating methods. In the case of lase heating method a laser with long wavelength i.e. IR or near IR lasers (e.g. $\mathrm{Co}_{2}$ and $\left.\mathrm{Nd}: \mathrm{YAG}\right)$ is used to increase the sample absorption and generate high temperature up to $7000{ }^{\circ} \mathrm{C}$. However, In the case of the resistive heater, either an external or an internal heater can be utilized to reach up to $1000{ }^{\circ} \mathrm{C}$. 

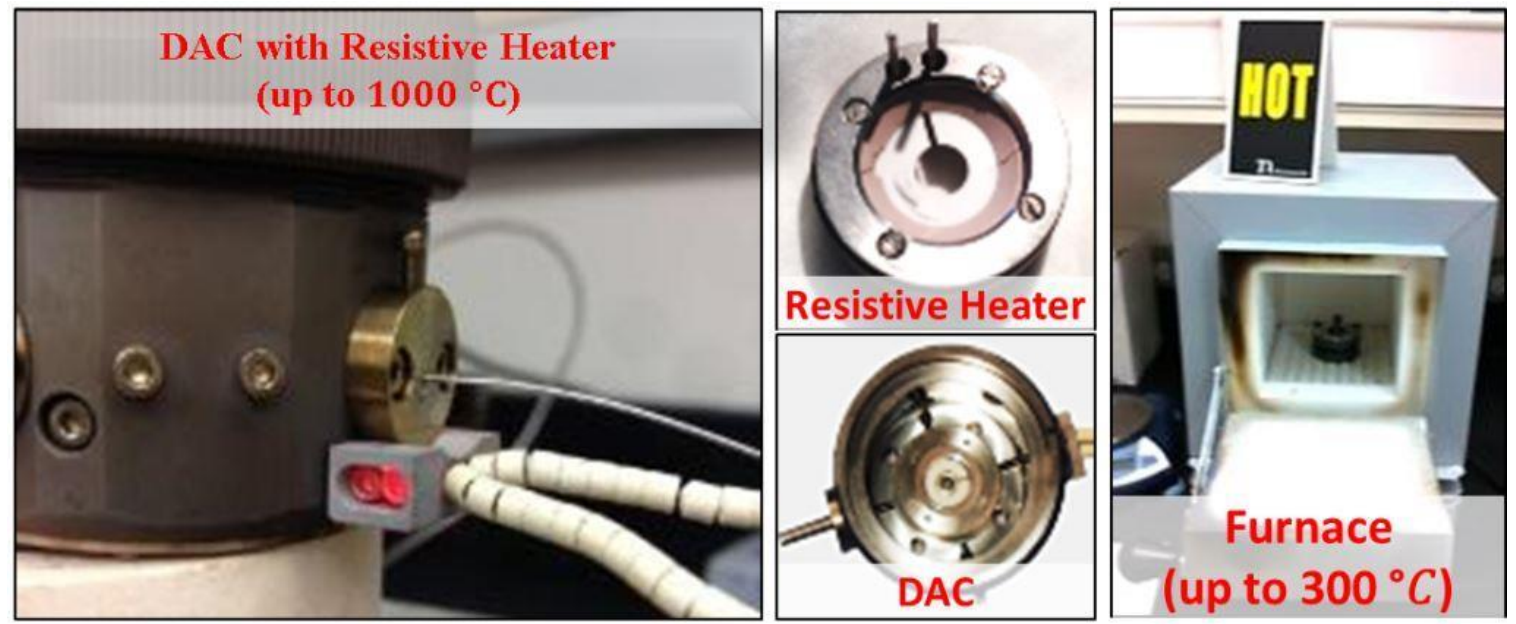

Figure 2.10 | Internal Resistive Heater DAC System and the External Furnace that we used for temperature below $300{ }^{\circ} \mathrm{C}$.

At Conn Center we use the Diacell HeliosDAC which is a membrane driven DAC that uses an internal resistive heater for generating temperatures up to $1000{ }^{\circ} \mathrm{C}$. A reducing gas mixture $\left(2 \% \mathrm{H}_{2}+98 \% \mathrm{Ar}\right)$ should flow through the DAC to prevent oxidation of the cell components.

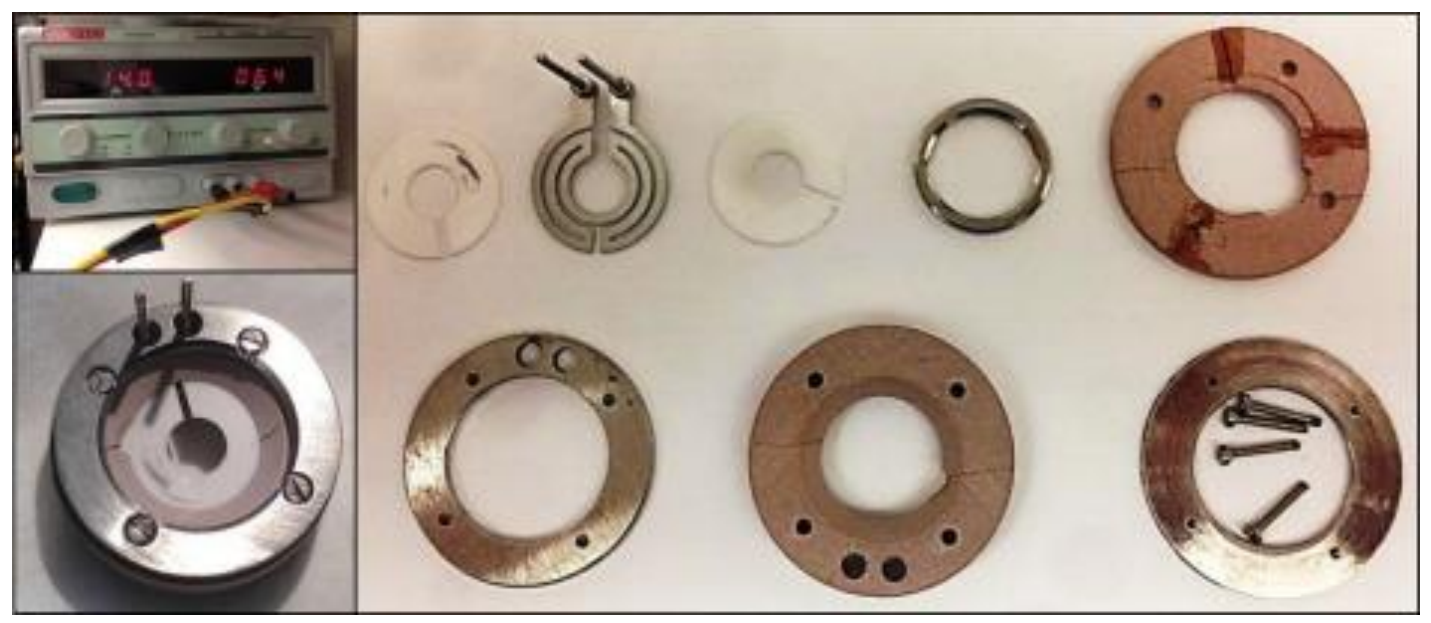

Figure 2.11 | Compact resistive heater $(80 \mathrm{~W})$, heater components, and DC power supply. 


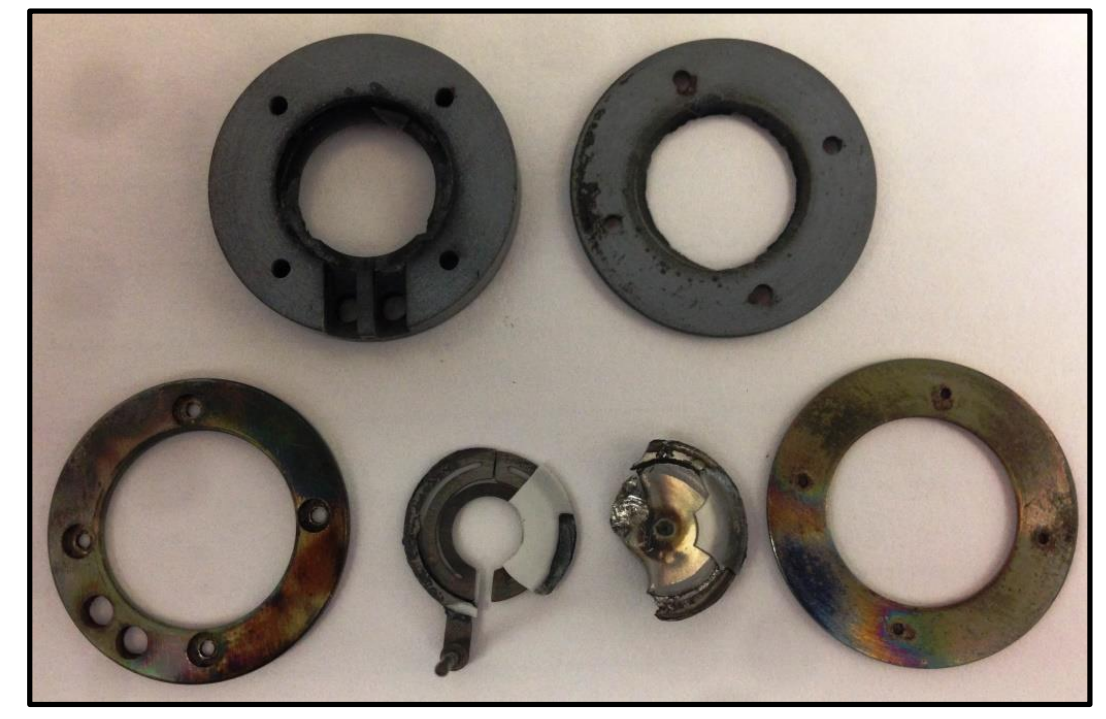

Figure 2.12| Burned heater components due to excess of current into the metal resistor.

Traditionally resistive heating experiments used wires wrapped around the gasket or backing plates, through which a current is passed to generate heat. The DAC itself can become quite hot during the heating process, which can be resulted in oxidation of the cell body. The membrane drive allows us to make pressure adjustments without disturbing the cell during in situ measurement.

\subsubsection{Pressure Measurement in the DAC}

XRD of a material with known equation of state and fluorescence spectroscopy of ruby are two main methods of pressure measurement inside the DAC. Nowadays, R2 line shift in Ruby PL is the most popular technique for measuring high pressures in DAC studies. Among the tested material, ruby has shown the most promising results. Ruby's luminescence had been vastly investigated in the fifties and sixties. The energy levels were calculated as a function of pressure. The two peaks in Figure 2.13 represent the energy levels of $\mathrm{Cr}$ in Ruby. They increase linearly with raising the pressure [126-129]. 
Over the several decades, different scientist groups have offered various calibrations for the ruby scale $[128,130-132]$. Following calibration of ruby PL against the pressure was calculated by Syassen [130]:

$$
\begin{gathered}
P=1870 \eta[1+5.9 \eta] \\
\eta=\frac{\lambda}{\lambda_{0}}-1
\end{gathered}
$$

Where $\mathrm{P}$ is the pressure, $\lambda$ and $\lambda_{0}$ are the wavelengths of the $\mathrm{R} 1$ line of ruby PL peak at the pressure $\mathrm{P}$, and the ambient pressure respectively.
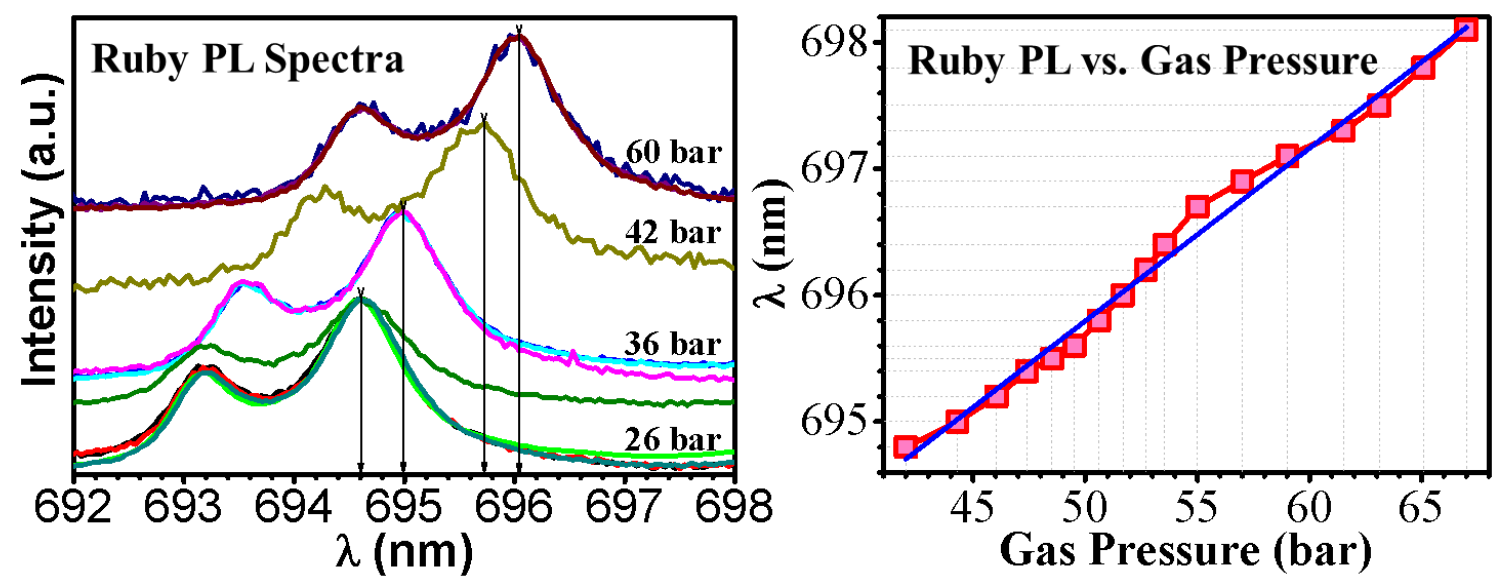

Figure 2.13 | Right: Ruby Photoluminescence Spectrum undergo a Red-Shift by Increasing the Pressure. Right: The gas pressure in the membrane of the DAC can be calibrated to be a scale of the sample pressure. 


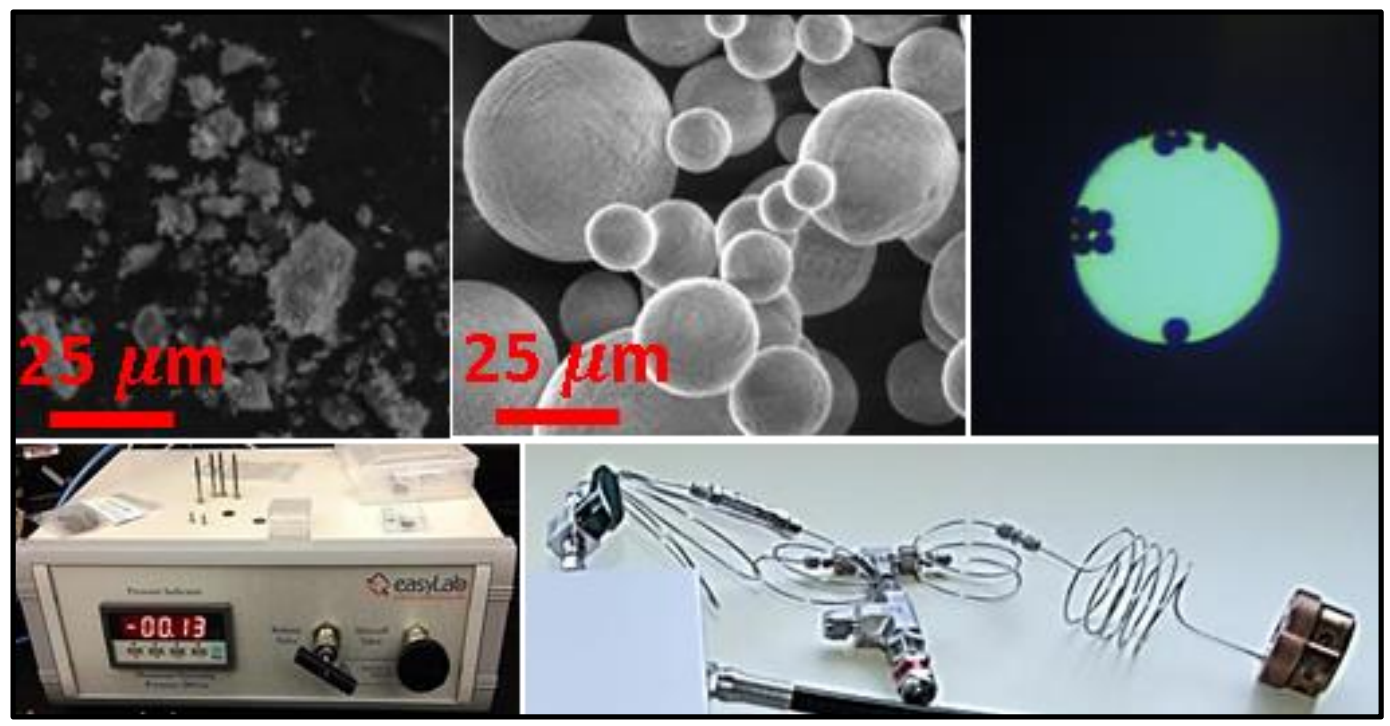

Figure 2.14 | Top Row: SEM Images of Two different Ruby Chips and Photograph of Ruby Chips inside Gasket Hole. Bottom Row: The Gas Controller and Gas Membrane along with the DAC.

\subsection{Characterization Equipment}

Various material characterization methods have been used in the presented work in this thesis. Theses techniques include Raman spectroscopy, Photoluminescence spectroscopy, UV/Vis/NIR absorption, X-ray photoelectron spectroscopy (XPS), X-ray diffraction (XRD), Transmission Electron Microscopy (TEM), Scanning Electron Microscopy (SEM), and Atomic force microscopy (AFM).

\subsubsection{Spectroscopy}

In general, spectroscopy involves obtaining information from the interaction of electromagnetic radiation with a physical system. Other modes of spectroscopy that do not require radiation like force spectroscopy, which can be performed using an atomic force microscope, are not as widely referred as spectroscopy. 
Different spectroscopy methods deal with various interactions between a sample and electromagnetic waves. For instance, Raman spectroscopy studies the photons scattered from the sample and vibrational modes of molecules. Also, various wavelengths can provide information about a range of atomic and molecular transitions presented in the following table:

Table 2.1 | The Wavelength Ranges and Sources of the Radiation.

\begin{tabular}{|l|l|}
\hline \multicolumn{1}{|c|}{ Source of radiation } & \multicolumn{1}{c|}{ Radiation type } \\
\hline nucleus & $\gamma$-rays \\
\hline core-level electrons & X-rays \\
\hline valence electrons & Ultraviolet (UV) \\
\hline valence electrons & Visible (Vis) \\
\hline molecular vibrations & Infrared (IR) \\
\hline $\begin{array}{l}\text { molecular rotations; } \\
\text { electron spin }\end{array}$ & Microwave \\
\hline nuclear spin & Radio waves \\
\hline
\end{tabular}




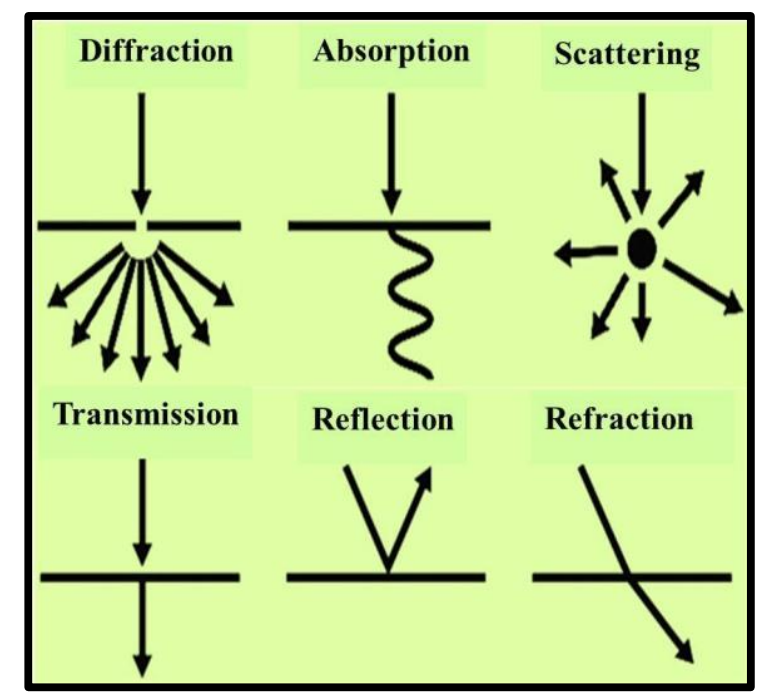

Figure 2.15 | The sketch shows different forms of energy exchange between a photon and the Sample.

\subsubsection{Raman Spectroscopy}

Raman spectroscopy involves illuminating a sample with laser beam and collecting the scattered light. Raman spectrometer detects the Raman shifts in the sample. Raman shift is the change in the wavenumber of the emitted photon with respect to wavenumber of the laser source. Each material has a specific Raman shift. Therefore, detecting the shift provides essential information for identifying a sample.

C.V. Raman was awarded the Noble prize in 1930 for discovering the Raman Effect that got more popular after the discovery of lasers in the sixties. When a laser beam irradiates a sample, most of the photons scatter elastically while a small percentage undergoes inelastic scattering.

Studying the energy of inelastic scattered photons is the subject of Raman spectroscopy. Elastic scattering or Rayleigh scattering refers to the case where the emitted and scattered photons have equal wavelength/frequency. 
In Raman spectroscopy, an incident photon creates an electron-hole pair. Either the hole or the electron emits or absorbs a phonon. Then they recombine and create a photon. Finally, the system restores the ground state with an additional phonon. If the energy of the absorbed photon is larger/smaller than that of the scattered one, the process is called stokes/anti-stokes. The Stokes processes can be observed only at low temperatures.

\subsubsection{Raman Applications in Material Science}

In material science, Raman scattering is mostly used to identify crystalline or polycrystalline insulators. In addition, some amorphous materials can be recognized by their specific bands (instead of peaks) like quartz. Raman spectroscopy is a powerful method for analyzing layered materials (even if they are metallic). In many cases, it can be utilized to determine the number of layers.

In the case of heavily doped semiconductors, Raman spectrum can be used to obtain electronic density of states. Raman scattering has also been used to study the growth of quantom dots in polar and non-polar planes of crystal structures.

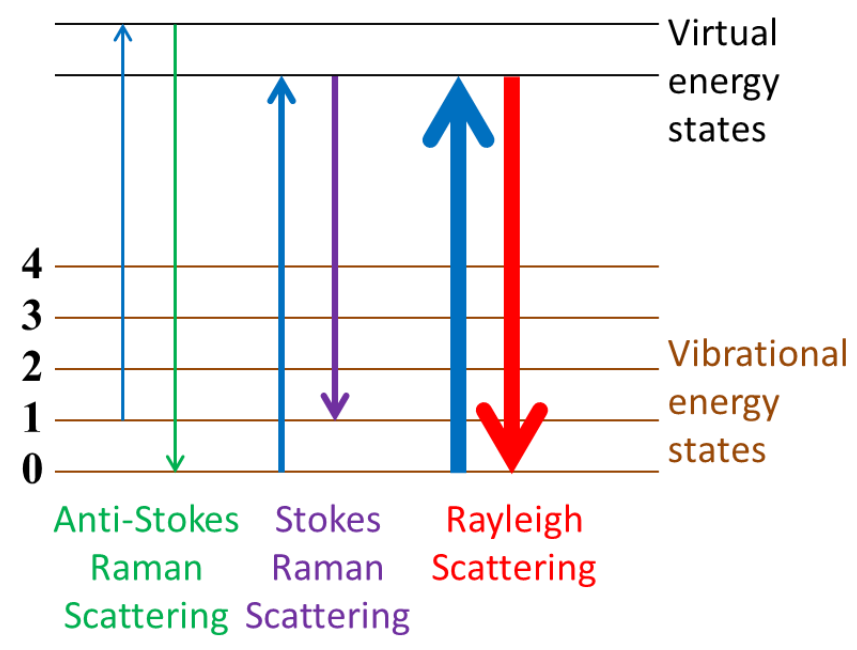

Figure 2.16 | Raman spectra energy states diagram. 


\subsubsection{Photoluminescence Spectroscopy}

In photoluminescence (PL) spectroscopy, a laser beam is shined on the sample. The incident photons excite the electrons to higher energy levels. The phote-excitation of electrons is followed by a spontaneous emission.

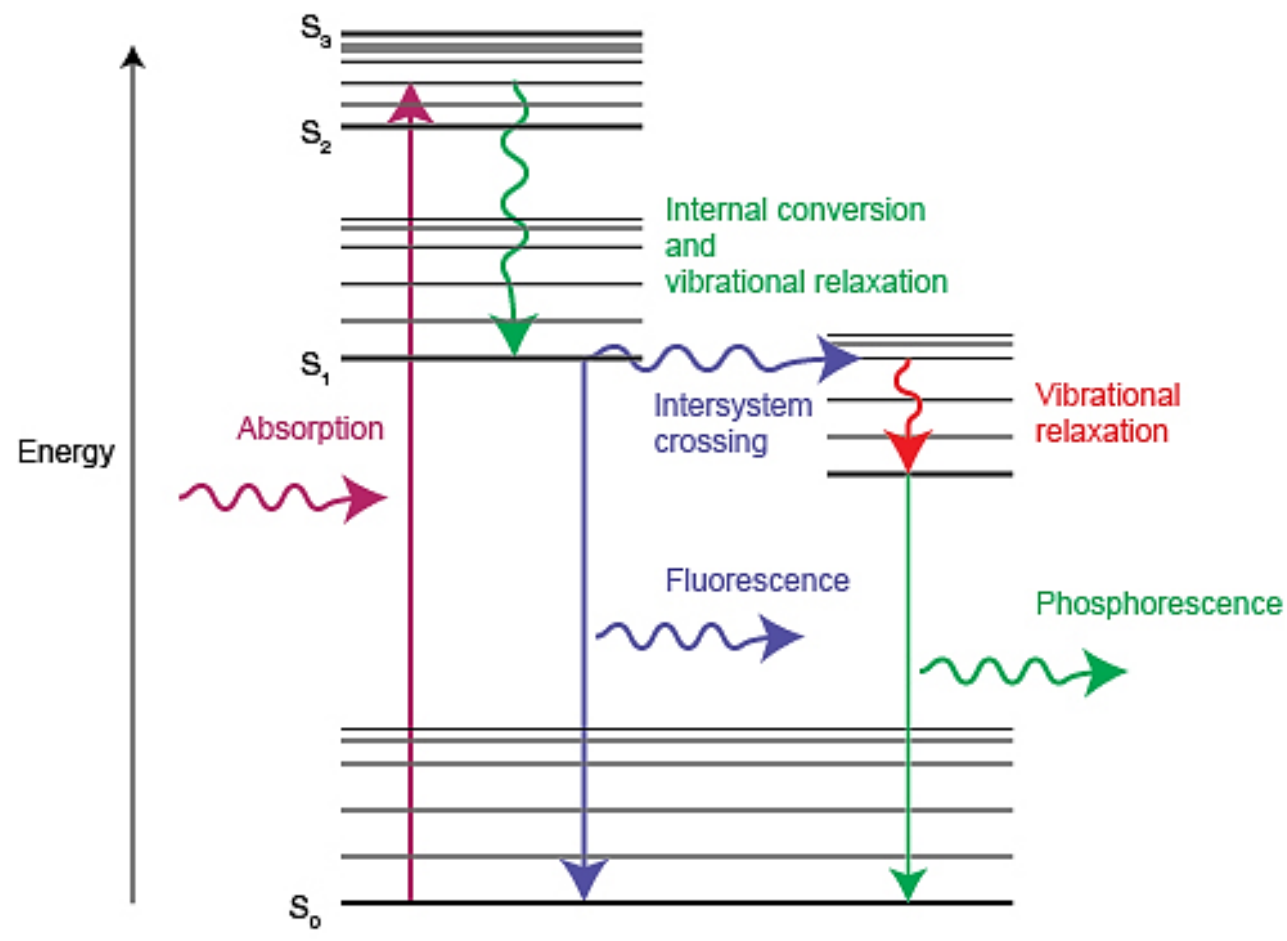

Figure 2.17 | Photoluminescence energy states diagram. 

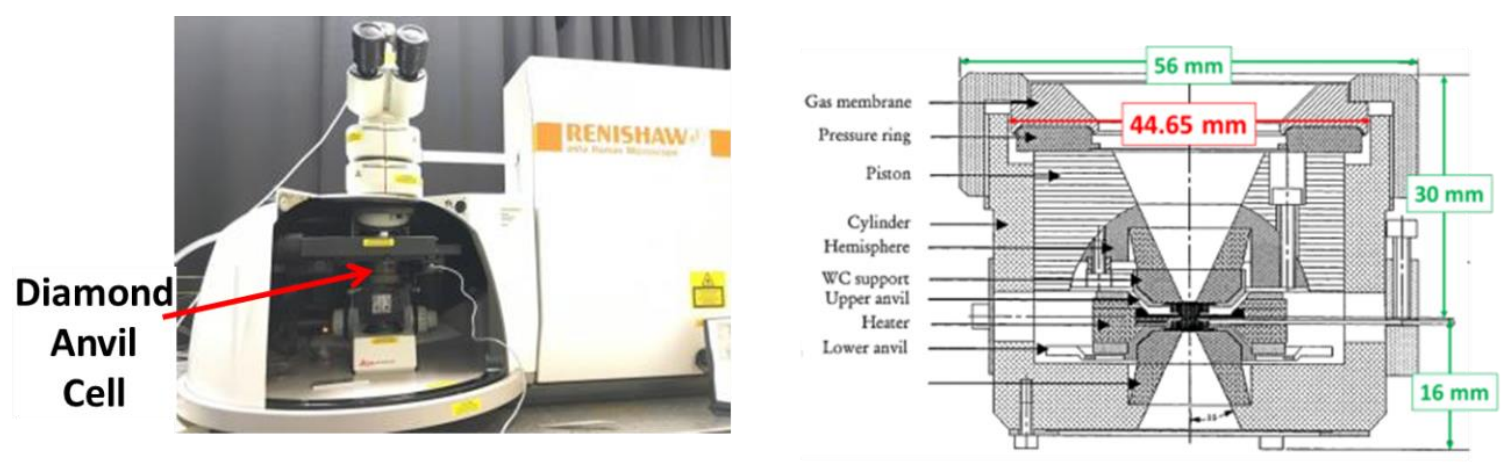

\section{Microscope Objective Lens}

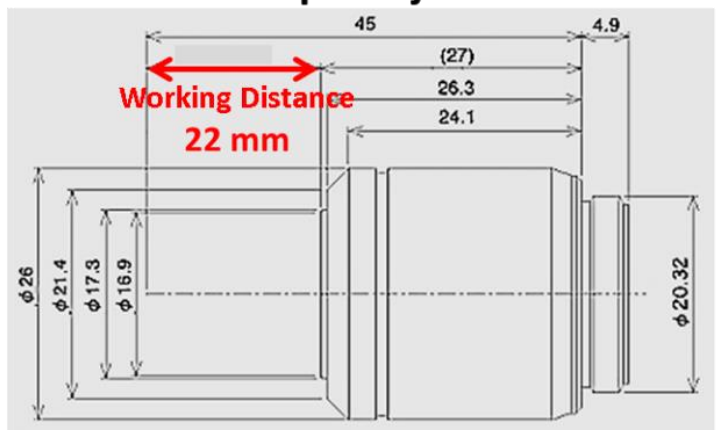

Figure 2.18| Our modified Raman and PL system for in situ high-pressure measurements.

\subsubsection{UV/Vis/NIR Absorption}

The energy of a molecule is composed of the energy of the electrons, vibrational energy of its atoms or rotational energy of the molecule itself. Since the electronic energy states are widely spaced, a high-energy beam is required to excite the molecule from one level to another. The complex substances have closer energy states and can absorb photons in near ultraviolet or visible region. The spacing between vibrational energy states is closer than electronic states and corresponding absorption happens in infrared region. Usually, the absorption peak obtained with spectrometer is a broad spectrum and includes all the three electrical, vibrational and rotational energy transitions.

The UV-VIS spectrometer provides the absorbance wavelength of a sample. Then the

energy of the compound is given by $E=h c / \lambda$ where $\mathrm{E}=$ energy, $\mathrm{h}=$ Planck's constant, 
$\mathrm{c}=$ speed of light, and $\lambda=$ wavelength. In addition, if either of extinction-coefficient or molar-absorptivity is known, one can calculate the other one by applying the Beer's law

$$
A=\varepsilon b c
$$

Where A is the measured absorbance, $\varepsilon$ is the molar extinction coefficient and $\mathrm{b}$ is the path length, and $\mathrm{c}=$ concentration.

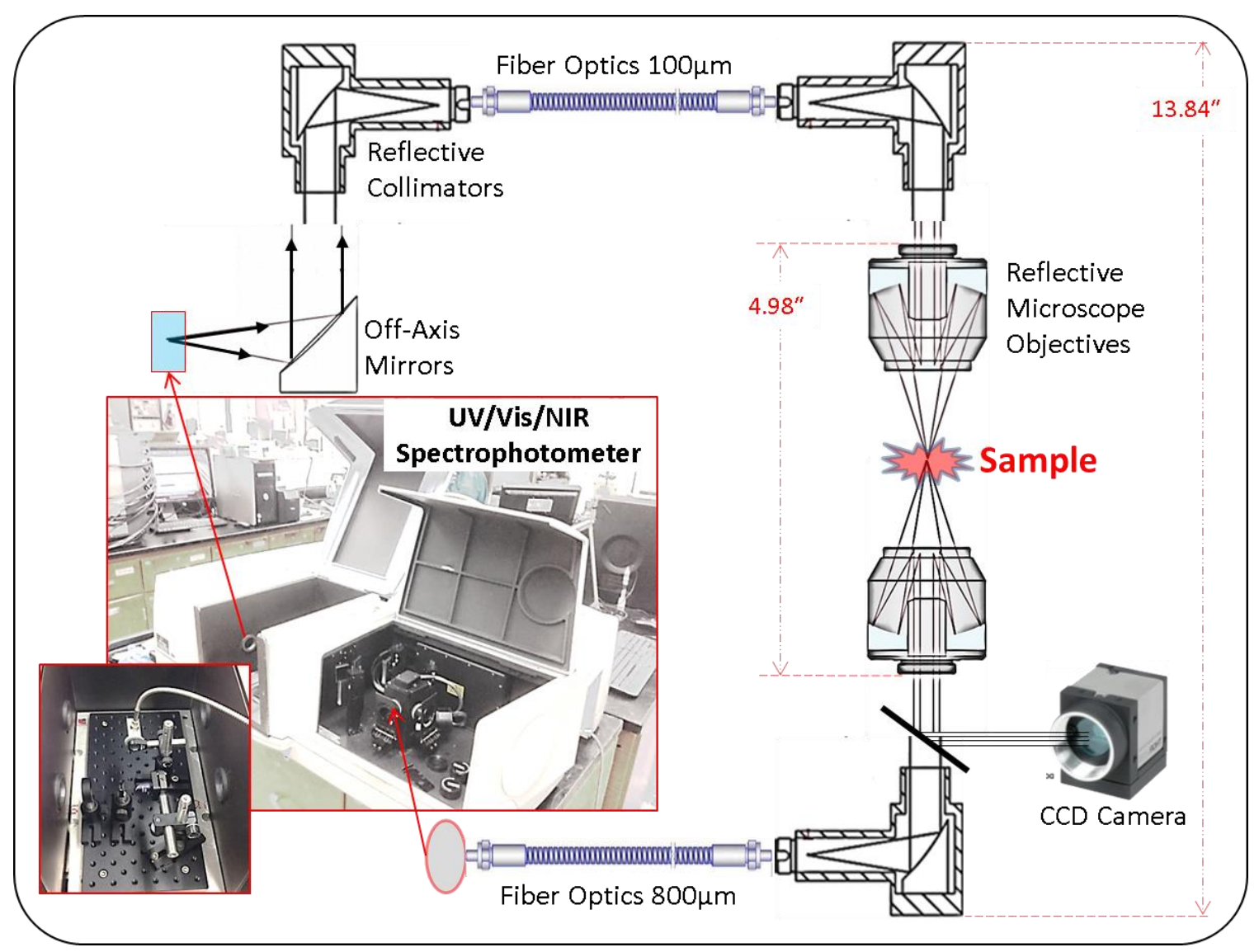

Figure 2.19 | Our developed optical setup for in situ high-pressure UV/Vis spectroscopy. 


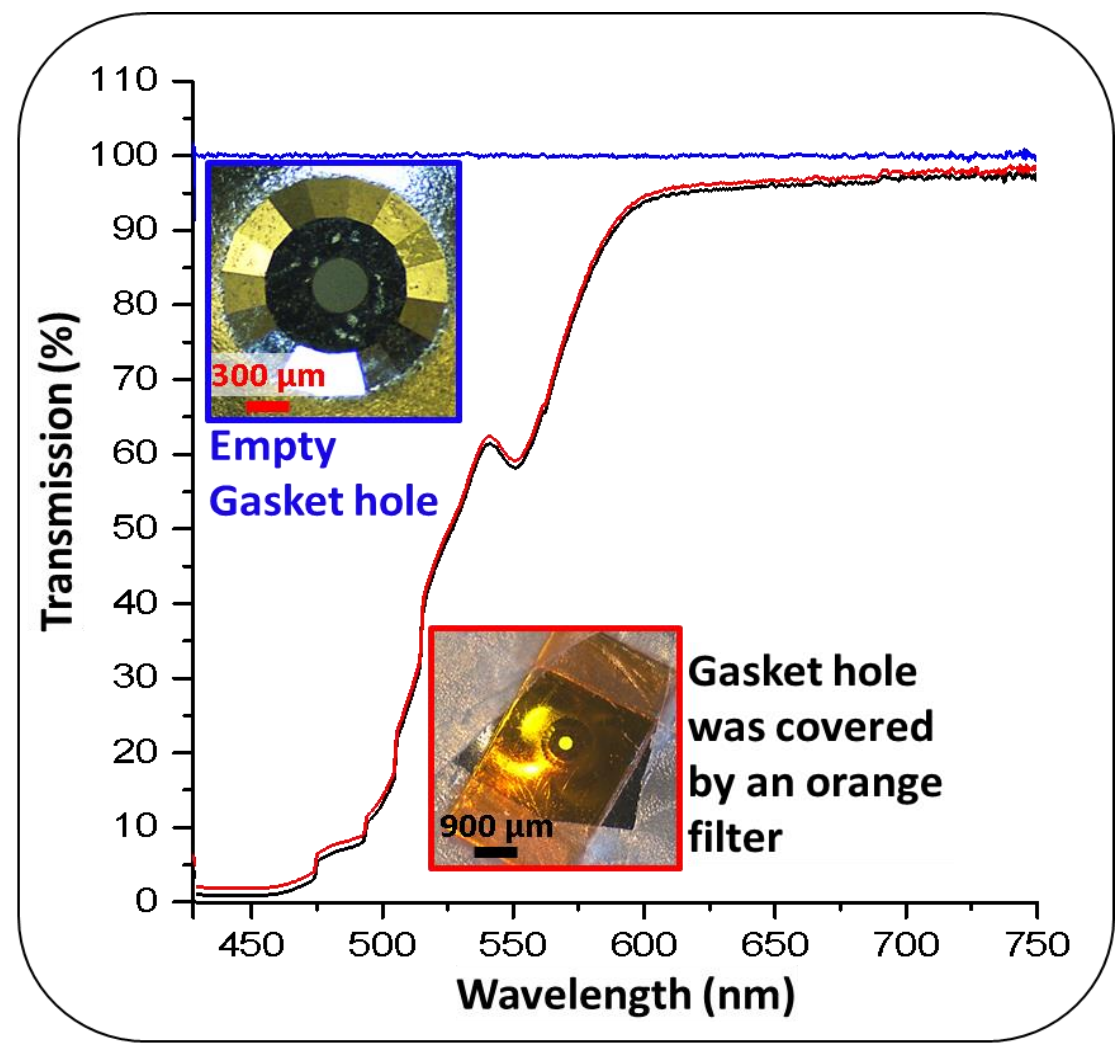

Figure 2.20| Testing Optical Setup for In Situ High-Pressure UV/Vis Spectroscopy. Transmission Absorption Spectra of a Gasket Hole (200 $\mu \mathrm{m}$ in diameter) Covered by Orange Filter.

\subsubsection{Powder X-ray diffraction (XRD)}

$\mathrm{XRD}$ is a valuable method for identifying unknown crystalline material. It also provides information on unit cell dimension. Since the x-rays wavelength is comparable to the size of atoms, they provide a useful tool to study the structural arrangement of atoms and molecules in a crystal. When X-rays illuminate a crystalline sample, arrays of atoms reflect the x-ray waves. The reflected waves may interfere in a constructive or destructive manner. In case of constructive interference, Bragg's law is used to calculate the spacing "d" between diffracting planes. 


$$
\mathrm{n} \lambda=2 \mathrm{~d} \sin \theta
$$

Where $\mathrm{n}$ is an integer, $\lambda$ is the $\mathrm{X}$-ray wavelength, and $\theta$ represents the scattering angle. If the wavelength of reflected X-rays did not change, the scattering is called elastic. Elastic scattering provides information on electron distribution. In contrast, in the inelastic scattering, some of the energy is transferred from X-ray photons to the electrons. The inelastic process can be used to probe the excitations of a sample.

The X-ray diffractometer consist of X-ray generator, sample holder and X-ray detector. X-ray is mostly generated in laboratories using X-ray tubes. In a cathode ray tube, a filament is heated to generate electrons. Then applying a high voltage accelerates the electrons toward the sample. A goniometer rotates the sample under the incident X-ray at an angle $\theta$ while, the detector rotates at $2 \theta$. Sometimes, the crystal structure of the sample can be improved through annealing before performing XRD. The typical data obtained with XRD consist of a plot that shows X-ray intensity versus the angle $2 \theta$ ranging from $5^{\circ}$ to $70^{\circ}$.

\subsubsection{X-ray Photoelectron Spectroscopy (XPS)}

X-ray Photoelectron Spectroscopy (XPS), is a powerful surface analysis technique. In this method, X-ray beams illuminate the sample leading to ionization and consequently emission of inner shell electrons. The kinetic energy of emitted electrons is measured at a distance of 1-10 nm from the sample. Since each element has a specific binding energy corresponding to each atomic orbital, the data can be used to probe the electronic state and composition of the sample surface. XPS spectrum demonstrates the rate of electron emission at each kinetic energy. Each peak indicates the presence of a particular element and the peak intensity is proportional to the element concentration in the probed region. 
Since the energy of incident beam $(h v)$ is known, the binding energy $(B E)$ can be calculated using the following equation:

$$
K E=h v-B E-\Phi
$$

Where $K E$ is the measured kinetic energy and $\Phi$ represents the work function of emitted electrons. The binding energy can be altered depending on the local environment of atoms and the oxidation state. For instance, the binding energy is larger for the atoms with greater positive oxidation state. Thus, XPS can be employed to distinguish various oxidation states and chemical surroundings.

\subsubsection{Scanning Electron Microscopy (SEM)}

Scanning electron microscope is a tremendously beneficial tool for studying the surface of a sample as it overcomes the resolution limit of the optical microscope. SEM uses a focused beam of electrons to scan the sample. The electrons are produced using an electron gun at the top of SEM column. A filament is heated to a temperature of 2000$2700 \mathrm{~K}$ that leads to the thermionic emission of electrons. A series of electromagnetic lenses are used to focus the electron beam on the sample. The interaction between the sample and the electron beam results in elastic and inelastic scatterings. The most common SEM mode to image the surface topography works by detecting inelastically scattered electrons called secondary electrons. Secondary electrons are low energy particles emitted by the atoms located near the sample surface.

The other common mode is imaging by backscattered electrons. They are scattered elastically from nuclei without losing energy. This method has a greater penetration depth and is less sensitive to the sample surface comparing with secondary electrons. However, this mode provides useful information about the atomic mass of the nuclei. 
Another possible form of emission from targeted sample is the radiation of high-energy X-ray photons. This method is referred as energy dispersive analysis of X-ray (EDAX) and is used to probe the elemental composition of a sample.

\subsubsection{Transmission electron microscope (TEM)}

TEM also uses an electron beam to image a sample. Similar to SEM, an electron gun is used as the beam source. Then, the electromagnetic lenses focus the beam on the sample. A portion of the electrons transmits through the sample while the others undergo scattering. TEM detects the transmitted electrons using a fluorescent screen to form the image. Thicker parts of the sample or regions with higher atomic density appear darker due to electron absorption, while the thinner parts appear brighter. In this mode, an aperture can be used to block the scattered electrons. The transmission intensity depends

on the electron transparency and thickness of the sample. This signifies the importance of sample preparation in TEM imaging. The sample thickness should be below $100 \mathrm{~nm}$, which limits the choice of samples.

In the case of crystalline samples, electrons scatter according to Bragg's law and construct a diffraction pattern. The diffraction intensity depends on the orientation of atomic planes with respect to the electron beam. Detecting the elastically scattered electrons through the electromagnetic lenses results in a pattern composed of spots. Each spot represents a specific atomic plane. This method provides information on the phase, atomic arrangements, and planes orientation. 


\subsubsection{Atomic Force Microscope (AFM)}

Atomic force microscopy is a method to study the topography of the sample by detecting the forces between the sample and AFM tip. The tip is attached to a shiny cantilever. The forces between sample and the tip result in bending of the cantilever. The backside of the cantilever is illuminated by a laser beam. The reflection from the cantilever is detected with a photo-diode. Small displacement or bending of the cantilever moves the beam on the photodiode. The image is formed according to the movement of the beam. Most AFM operate in two different modes; tapping mode and contact mode.

In tapping mode, a voltage is applied to the cantilever causing it to oscillate at its resonance frequency. Forces applied by the sample affect the frequency. In this mode, the image is created by detecting the frequency variations. The software also provides images formed by detecting the amplitude and phase of the cantilever oscillations. The phase image demonstrates the changes in sample material. AFM in contact mode operates by moving the sample under the tip so that the forces between these two remain constant. The movement of the sample or the tip is controlled with a piezo-electric ceramic attached to the AFM scanner.

AFM is a precise method for measuring the thickness and particle size at the surface. The AFM tip can be replaced with a magnetic tip to image the magnetic properties of the sample. 


\subsection{Synthesis Method}

\subsubsection{Rhombohedral $\alpha-\mathrm{AgGaO}_{2}$}

These synthesis experiments were conducted using "Diacell HeliosDAC" diamond anvil cell (DAC) (Almax EasyLab Inc.). The high-pressure cell was equipped with an internal resistive heater with capability to heat up the sample to the temperatures of up to 1000 ${ }^{\circ} \mathrm{C}$. To measure the temperature a thermocouple (K-type) was used which placed close to the diamond culet and sample chamber. Diamond anvils with $820 \mu \mathrm{m}$ flat culets were used in the cell and samples were loaded into a $250-\mu \mathrm{m}$ hole of Inconel gasket. To calibrate the pressure, we utilized the R1-luminescence line shift of ruby crystal under the pressure. A mixture of hydrogen (2\%) and argon (98\%) gas was used to avoid cell oxidation during the heating process. The experiment duration for each synthesis process was two hours. Synthesis of $\alpha-\mathrm{AgGaO} 2$ was possible at temperatures from $480{ }^{\circ} \mathrm{C}$ to 620 ${ }^{\circ} \mathrm{C}$ and pressures between 1.5 and $10 \mathrm{GPa}$. Twenty samples including several control samples were prepared. The precursors were $\mathrm{Ag}_{2} \mathrm{O}$ and $\mathrm{Ga}_{2} \mathrm{O}_{3}$ powders mixed at various weight ratios. Some of the control samples were prepared using pure $\mathrm{Ag}_{2} \mathrm{O}$ or $\mathrm{Ga}_{2} \mathrm{O}_{3}$ powders. The influence of high-pressure only and high temperature only on mixture of $\mathrm{Ag}_{2} \mathrm{O}$ and $\mathrm{Ga}_{2} \mathrm{O}_{3}$ powder was monitored during control experiments. All the samples were characterized using different techniques including Raman spectroscopy with red laser $(633 \mathrm{~nm})$ and Renishaw inVia Raman/PL microscope, scanning electron microscopy (SEM), transmission electron microscopy (TEM), selected area electron diffraction (SAED) and energy dispersive X-ray spectroscopy (EDX). TEM samples were prepared by dispersing powders on dedicated $3.05 \mathrm{~mm}$-diameter, 300 mesh copper grid-supported amorphous lacey carbon films. In our synthesis experiments of $\alpha$ - 
$\mathrm{AgGaO}_{2}$, mixtures of the following two commercially available oxide powders were used as the precursors:

A. Silver (I) Oxide ( $\left.\mathrm{Ag}_{2} \mathrm{O}\right), 99.99 \%$ (metals basis) from Alfa Aesar (CAS: 20667-123) and

B. Gallium (III) oxide $\left(\mathrm{Ga}_{2} \mathrm{O}_{3}\right), 99.99 \%$ (trace metal basis) from Acros Organics (CAS: 12024-21-4).

The precursors were loaded into a diamond anvil cell (DAC) where high-pressure and high-temperature conditions were produced. We carried out several experiments with the temperatures between $200{ }^{\circ} \mathrm{C}$ and $600{ }^{\circ} \mathrm{C}$ and pressures between $1 \mathrm{GPa}$ and $10 \mathrm{GPa}$. The DAC used for these experiments consisted of two diamonds with $0.82 \mathrm{~mm}$ culets. The gasket was made of $0.3 \mathrm{~mm}$-thick Inconel alloy foil and a $0.25 \mathrm{~mm}$-diameter hole served as the high-pressure reaction chamber. The loading of precursor samples into the DAC was performed under a stereo microscope with magnification power of $180 \mathrm{X}$. Experimental conditions and parameters of 20 samples prepared for this work, including 7 control samples are listed in Table S1 below. 
Table 2.2 | List of samples prepared for this work

\begin{tabular}{|c|c|c|c|c|c|c|c|}
\hline \multirow{2}{*}{ sample \# } & \multicolumn{3}{|c|}{ Precursor } & \multirow{2}{*}{$\begin{array}{c}\text { Temperature } \\
\text { ( C) }\end{array}$} & \multirow{2}{*}{$\begin{array}{c}\text { Pressure } \\
\text { (GPa) }\end{array}$} & \multirow{2}{*}{$\begin{array}{l}\text { Time } \\
(\mathrm{hrs})\end{array}$} & \multirow{2}{*}{$\begin{array}{c}\alpha-\mathrm{AgGaO} \mathrm{GaO}_{2} \\
\text { Raman peaks }\end{array}$} \\
\hline & $\mathrm{Ga}_{2} \mathrm{O}_{3}$ & $\mathrm{AgO}_{2}$ & molar ratio & & & & \\
\hline 1 & + & - & - & $\sim 25$ & $\sim 0$ & - & $x$ \\
\hline 2 & - & + & - & $\sim 25$ & $\sim 0$ & - & $x$ \\
\hline 3 & + & - & - & 600 & $\sim 0$ & 2 & $x$ \\
\hline 4 & - & + & - & 600 & $\sim 0$ & 2 & $x$ \\
\hline 5 & + & + & $1: 2$ & 600 & $\sim 0$ & 2 & $x$ \\
\hline 6 & + & - & - & 600 & 10 & 2 & $x$ \\
\hline 7 & - & + & - & 600 & 10 & 2 & $x$ \\
\hline 8 & + & + & $1: 1$ & 600 & 10 & 2 & $\checkmark$ \\
\hline 9 & + & + & $3: 1$ & 600 & 10 & 2 & $\checkmark$ \\
\hline 10 & + & + & $1: 3$ & 600 & 10 & 2 & $\checkmark$ \\
\hline 11 & + & + & $1: 2$ & 600 & 10 & 2 & $\checkmark$ \\
\hline 12 & + & + & $2: 1$ & 600 & 10 & 2 & $\checkmark$ \\
\hline 13 & + & + & $1: 1$ & 480 & 10 & 2 & $\checkmark$ \\
\hline 14 & + & + & $3: 1$ & 480 & 10 & 2 & $\checkmark$ \\
\hline 15 & + & + & $1: 1$ & 320 & 10 & 2 & $x$ \\
\hline 16 & + & + & $1: 1$ & 600 & 6 & 2 & $\checkmark$ \\
\hline 17 & + & + & $1: 1$ & 600 & 3 & 2 & $\checkmark$ \\
\hline 18 & + & + & $1: 1$ & 600 & 1.5 & 2 & $\checkmark$ \\
\hline 19 & + & + & $1: 1$ & 200 & 1.5 & 2 & $x$ \\
\hline 20 & + & + & $1: 1$ & 400 & 1.5 & 2 & $x$ \\
\hline
\end{tabular}

All mixed samples that were subjected to the pressure above $1.5 \mathrm{GPa}$ and a temperature above $400{ }^{\circ} \mathrm{C}$ show 4 Raman peaks characteristic of $\alpha-\mathrm{AgGaO}_{2}$ in the region $300 \mathrm{~cm}^{-1}$ to $700 \mathrm{~cm}^{-1}$. However, the signal was most intense for the samples synthesized under higher temperature and mixed in the 1:1 ratio. TEM analysis indicated that these samples had a relatively uniform phase. The only other Raman peaks were attributed to silver oxide or gallium oxide initial phases; and were present because of non-stoichiometric mixture due 
to a very small volume of samples for DAC experiments. This non-stoichiometric effect was also revealed in SEM and TEM study. In addition to crystallites of $\alpha-\mathrm{AgGaO}_{2}$, we also observed un-reacted grains of $\mathrm{Ag}_{2} \mathrm{O}$ or $\mathrm{Ga}_{2} \mathrm{O}_{3}$.

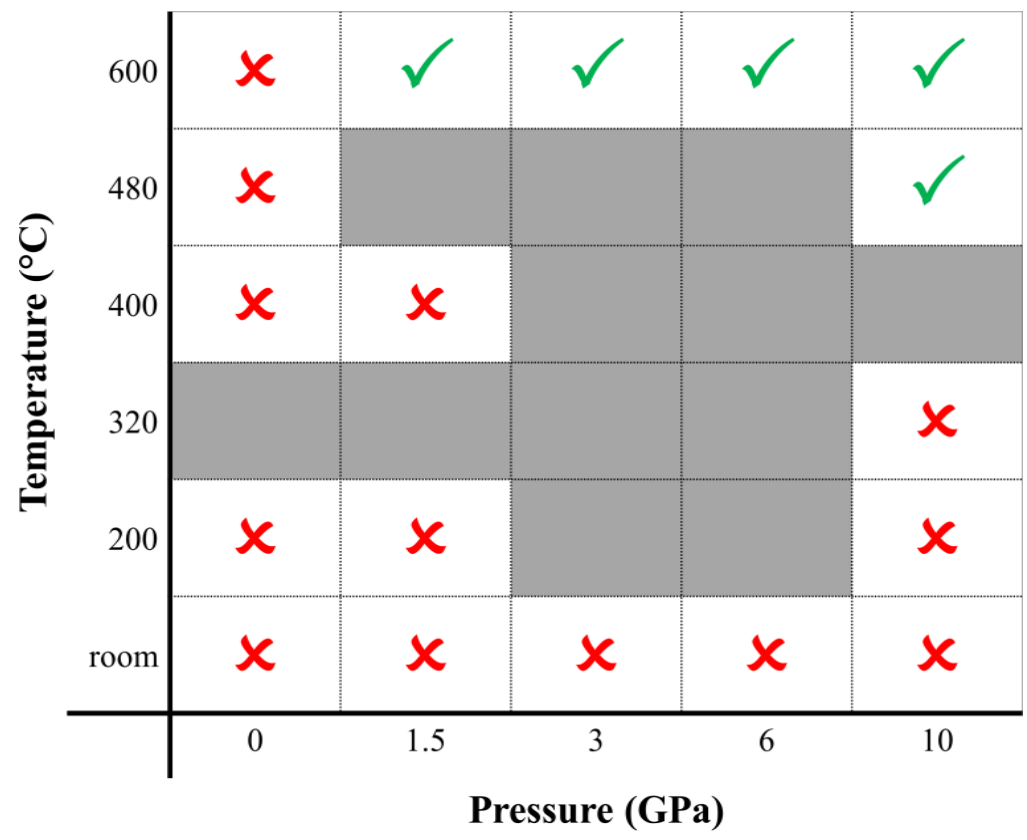

Figure 2.21 | Temperature-Pressure diagram representing conditions used in our DACbased $\alpha-\mathrm{AgGaO}_{2}$ synthesis experiments. Each square represents one experiment and its specific conditions. Green check mark denotes formation of rhombohedral $\alpha-\mathrm{AgGaO}_{2}$. Red crosses show experiments, which did not produce rhombohedral $\alpha-\mathrm{AgGaO}_{2}$.

\subsubsection{Synthesis Three-Dimensional (3D) Graphene Foam}
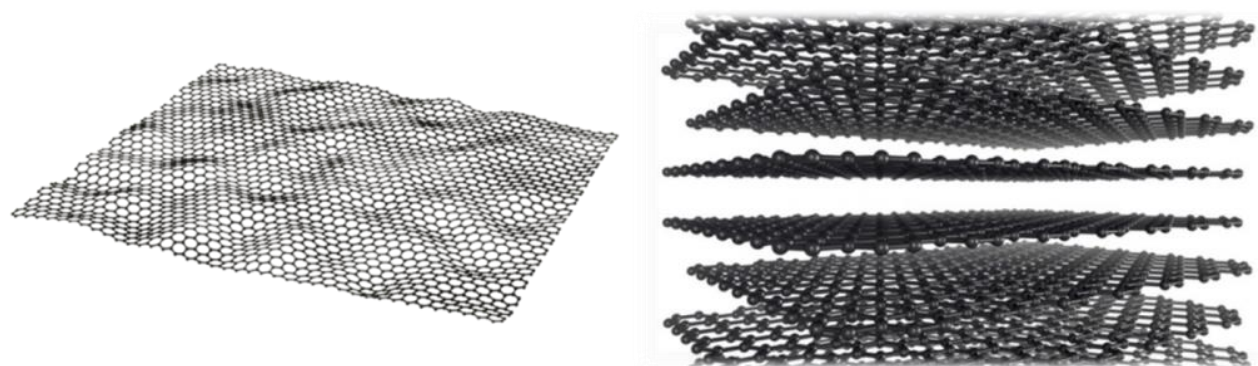

Figure $2.22 \mid$ Schematic image of single graphene sheet and graphite layers. 
In this work three-dimensional graphene was synthesized by pyrolysis of organic precursor, citric acid $\left(\mathrm{C}_{6} \mathrm{H}_{8} \mathrm{O}_{7}\right)$. Commercial $\mathrm{Ni}$ foams (Marketech International, Inc., Port Townsend, USA), $346 \mathrm{~g} / \mathrm{m}^{2}$ in surface density, $1.6 \mathrm{~mm}$ in thickness and $>99.9 \%$ purity were used to catalyze the graphene growth. Firstly, the nickel foams were washed and sonicating with ethanol and acetone for 10 min, respectively. Then they were immersed in a $5 \mathrm{M} \mathrm{HCl}$ acid solution for about 20 min to remove the oxide layer on their surface, and finally rinsed several times with deionized water before dipping to the concentrated citric acid solution. The nickel foams covered with citric acid were dried at $70{ }^{\circ} \mathrm{C}$ in an oven for about 6 hours. Secondly, the nickel foams transferred to the tube furnace for annealing at $1000^{\circ} \mathrm{C}$ in the presence of $\mathrm{Ar}$ and $\mathrm{H}_{2}$ for 10 min.

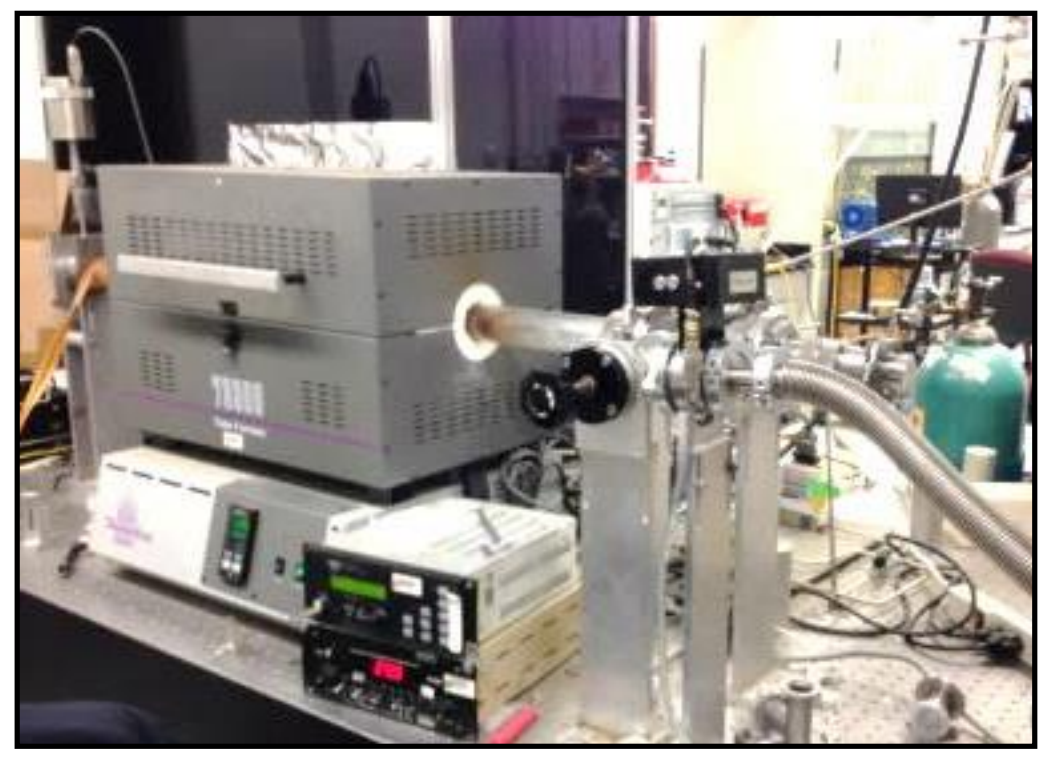

Figure 2.23 | Chemical Vapor Deposition System setup. It was used for both CVD and the developed pyrolysis methods. 


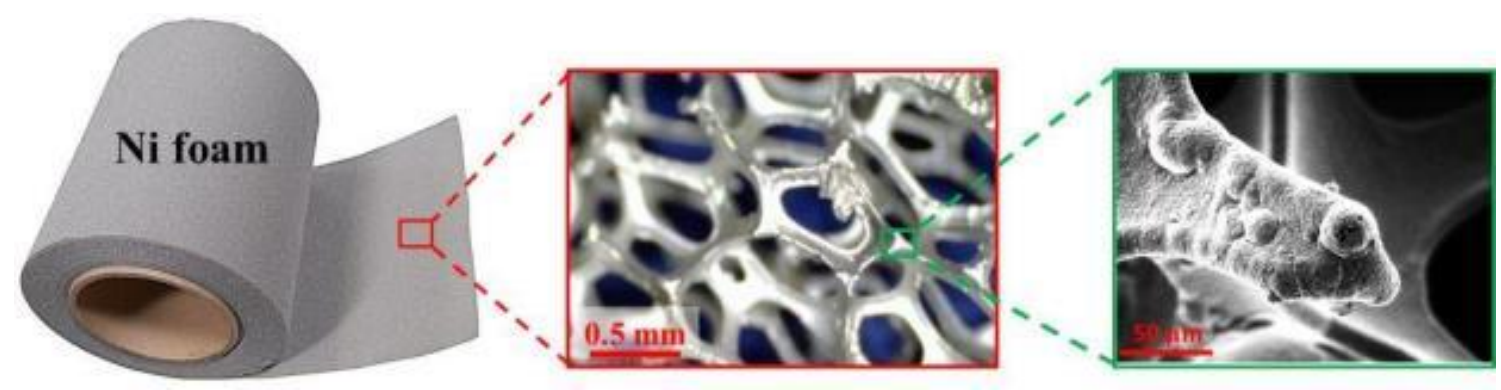

Figure 2.24 | Optical and scanning electron microscopy (SEM) images of nickel foam.

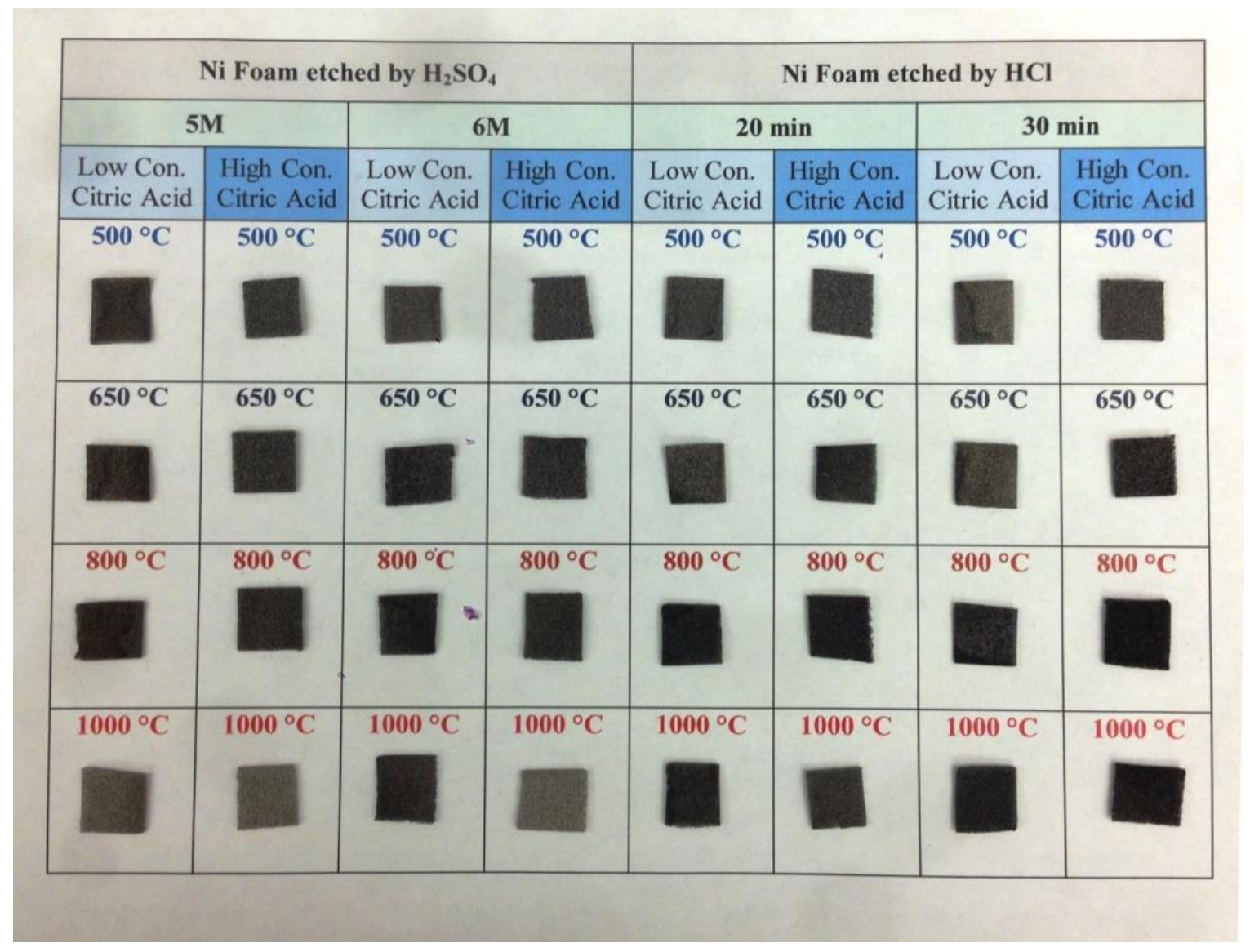

Figure 2.25 | Photograph of three-dimensional graphene foams fabricated at 500, 650, 800 , and $1000{ }^{\circ} \mathrm{C}$ temperature and with different pre-treatment of nickel surface. 


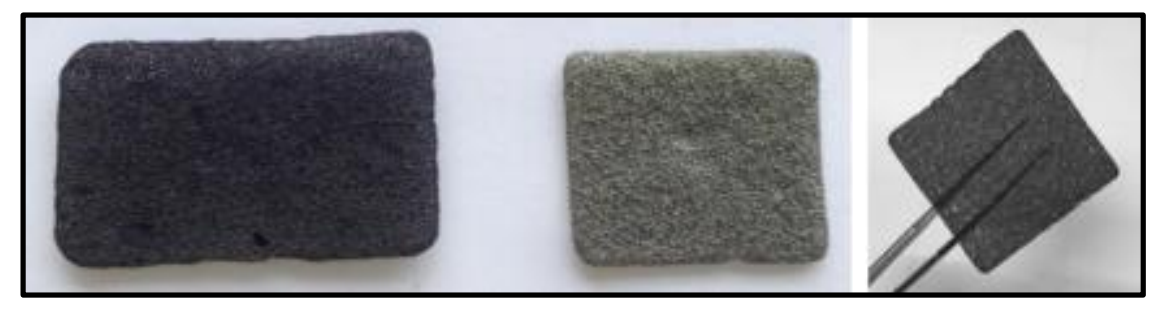

Figure 2.26 | Nickel foam (center), three-dimensional graphene before (left) and after (right) etching the nickel template.

The flow rates the gases $\left(\mathrm{H}_{2}: \mathrm{Ar}\right)$ were $20: 13 \mathrm{sccm}$, respectively. After $10 \mathrm{~min}$ of annealing, the sample was cooled by rate of $\sim 10{ }^{\circ} \mathrm{C} / \mathrm{min}$. Nitrogen doped graphene samples were synthesized the same method by adding nitrogen-containing organic materials to the solution of citric acid. In this work caffeine $\left(\mathrm{C}_{8} \mathrm{H}_{10} \mathrm{~N}_{4} \mathrm{O}_{2}\right)$, urea $\left(\mathrm{CH}_{4} \mathrm{~N}_{2} \mathrm{O}\right)$ and acetaminophen $\left(\mathrm{C}_{8} \mathrm{H}_{9} \mathrm{NO}_{2}\right)$ were used with citric acid solution as the source of carbon and nitrogen.

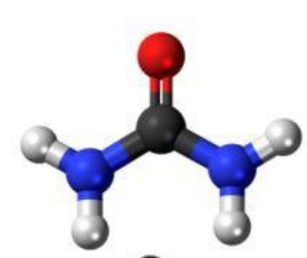<smiles>NC(N)=O</smiles>

\section{$\mathrm{CH}_{4} \mathrm{~N}_{2} \mathrm{O}$ Urea}

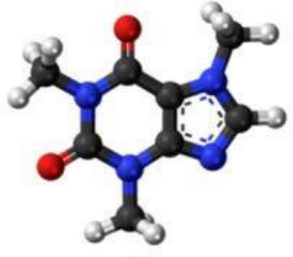<smiles>Cn1c(=O)c2c(ncn2C)n(C)c1=O</smiles>

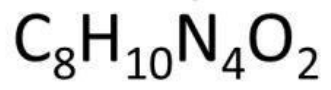
Caffeine
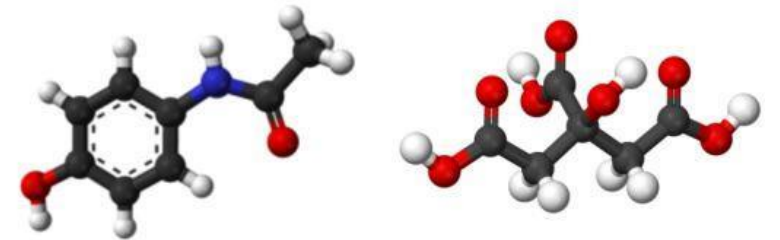<smiles>CC(=O)Nc1ccc(O)cc1</smiles><smiles>O=C(O)CC(O)(CC(=O)O)C(=O)O</smiles>

$\mathrm{C}_{8} \mathrm{H}_{9} \mathrm{NO}_{2}$

$\mathrm{C}_{6} \mathrm{H}_{8} \mathrm{O}_{7}$

Acetaminophen Citric acid

Figure 2.27 | Molecular structure and chemical formula of organic precursors used for graphene growth.

All solutions were made to be saturated with equal weight portions of the organic precursors. Raman spectroscopy data were collected using red laser and Renishaw inVia 
Raman/PL spectrometer. The samples were analyzed with a HeNe laser excitation of 633 $\mathrm{nm}$, using a 50X objective. Surface morphology characterization of the samples was examined using a scanning electron microscope.

\subsubsection{Synthesis of Phosphorene}

Few-layer black phosphorus or phosphorene can be produced by exfoliating the bulk black phosphorus. Due to the high cost of the black phosphorus, we decided to prepare this precursor. In this work, we prepared bulk black phosphorus using short way transport reaction based on the research work by Köpf et al. [133]. The research group leads by Dr. Nilges have been developed this synthesis method through series of improvements that have been published [133-135]. In this method, tin(IV) iodide $\left(\mathrm{SnI}_{4}\right)$, tin $(\mathrm{Sb})$, and red phosphorus are used as the precursors to synthesis the black phosphorus. Precursors annealed at $\sim 600{ }^{\circ} \mathrm{C}$ in high vacuum environment. For this purpose we used Pyrex glass tube with one open end and filled it with $10 \mathrm{mg}$ of tin(IV) iodide, $20 \mathrm{mg}$ of tin, and 400 mg of red phosphorus. Then, using the vacuum system shown in Figure 2.28 the Pyrex tube was evacuated to $\sim 10^{-6}$ Torr. To reach such a high vacuum $\left(\sim 10^{-6}\right.$ Torr $)$ we used a combination of two vacuum pumps, an oil diffusion pump and a mechanical (rotary vane) pump. The diffusion pump typically cannot start working from atmospheric pressure and need a backing (roughing) pump. 


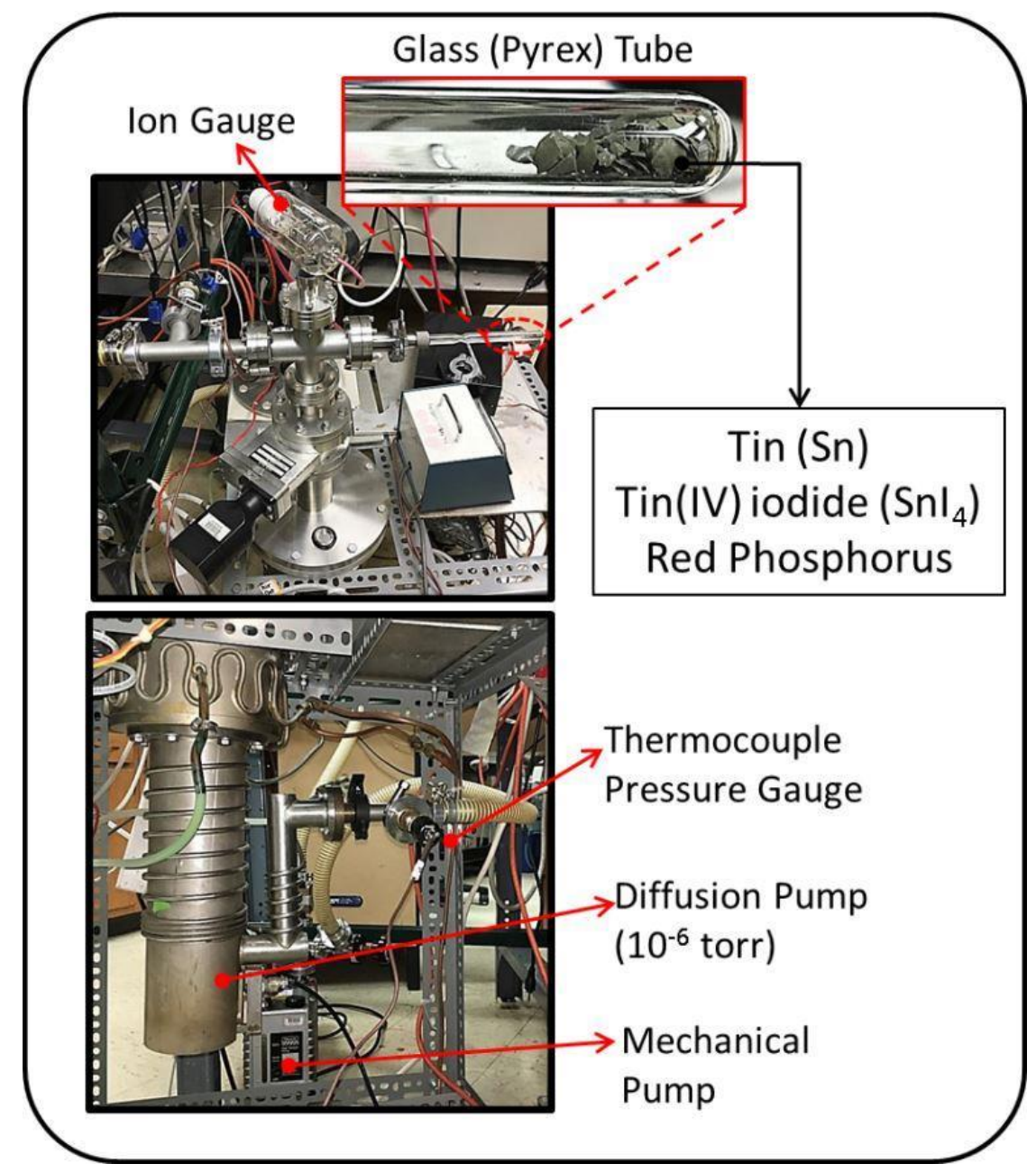

Figure 2.28 | Vacuum system for synthesis of black phosphorus by short way transport reaction methods.

Using an oxyacetylene torch the Pyrex tube was sealed and transferred to the furnace for the annealing process. The sealed Pyrex tube was placed in the furnace in a way that there was a temperature gradient $\left(\sim 40^{\circ} \mathrm{C}\right)$ between the two ends of the tube. This temperature gradient is necessary for the conversion process of red phosphorus to black phosphorus. The choice of the Pyrex glass compare to the quartz has some advantageous such as easier sealing process but the Pyrex critical point is close to the annealing 
temperature of the precursors $\left(\sim 600^{\circ} \mathrm{C}\right)$ which caused some difficulties in annealing process.

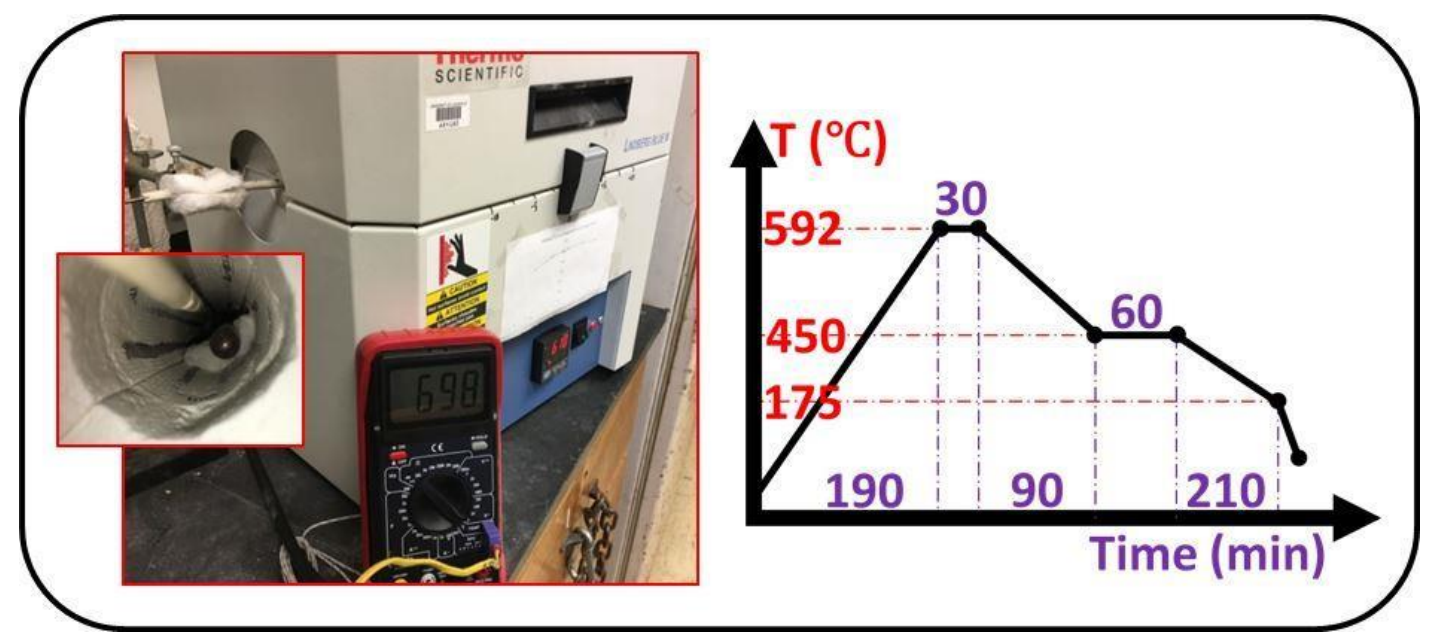

Figure 2.29 | The tube furnace and the temperature profile that was used to synthesis black phosphorus.

During the annealing process, first, the temperature was increased from room temperature to $\sim 600{ }^{\circ} \mathrm{C}$ in 190 minutes and the sample was kept in that temperature for 30 minutes. Then the temperature was decrease to $\sim 450{ }^{\circ} \mathrm{C}$ in 90 minutes. The samples were held at $450{ }^{\circ} \mathrm{C}$ for 60 minutes. Finally, the sample was gradually cooled down to the room temperature. At the end of the annealing process inside the Pyrex tube black phosphorus crystal was formed. To avoid degradation of the sample we did not expose the sample to open air instead, we removed the black phosphorus from the tube inside the Argon glove box.

The synthesized bulk black phosphorus was used to prepare few-layer black phosphorus through liquid phase exfoliated (LPE) method. In this work dimethylformamide (DMF) with chemical formula, $\mathrm{C}_{3} \mathrm{H}_{7} \mathrm{NO}$, was used as the solvent in the process. Sample crystal was suspended in the solvent and was sonicated for 2 hours. After 2 hours sonication the 
resulted samples were contained 1-5 layers. However, shorter time of sonication resulted to the samples with significantly more layers. In the next step the samples was placed in a centrifuge with 10,000 rpm for half an hour. During this step the temperature was maintained at about room temperature to avoid any side reaction. The centrifugation separated the phosphorene based on the number of layers in other words phosphorene samples with less number of layers were concentrated at top of the vial container and phosphorene with higher number of layers concentrated at bottom of the container.

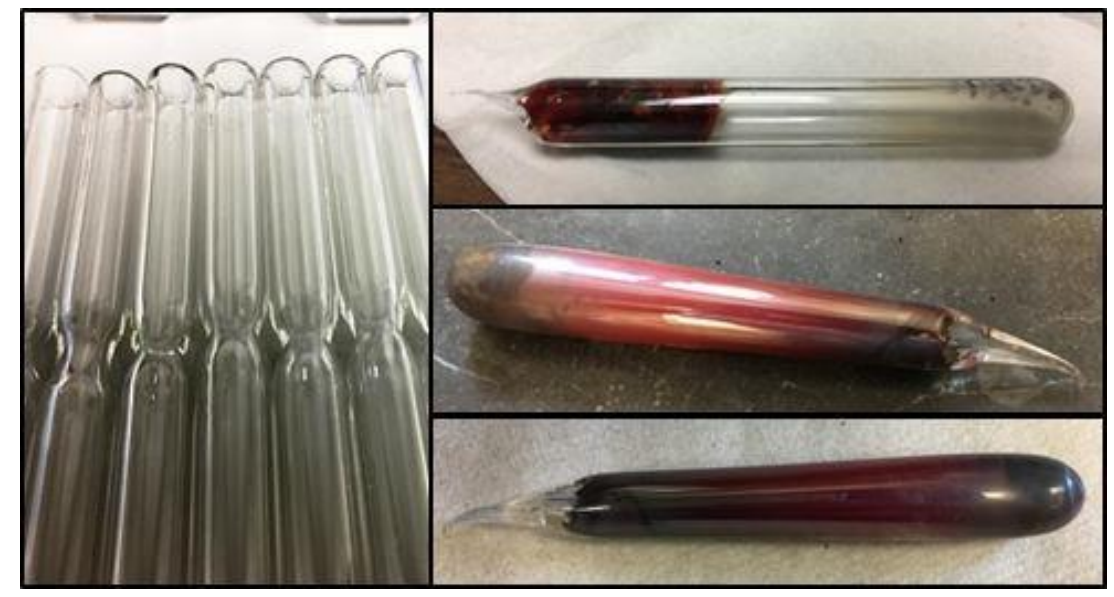

Figure 2.30 | Pyrex tubes before sealing and evacuated glass (Pyrex) tube after annealing at $600{ }^{\circ} \mathrm{C}$. 


\section{CHAPTER 3: RESULTS AND DISCUSSION}

\subsection{Rhombohedral $\alpha-\mathrm{AgGaO}_{2}$}

In this work [72], Raman spectroscopy was performed for all prepared samples. A representative set of acquired Raman spectra is shown in Figure 3.1. All the mixed $\mathrm{Ag}_{2} \mathrm{O}$ $\mathrm{Ga}_{2} \mathrm{O}_{3}$ powder samples that were annealed under high pressure in the DAC feature a group of peaks between $350 \mathrm{~cm}^{-1}$ and $700 \mathrm{~cm}^{-1}$. Intriguingly, none of the control samples demonstrated these peaks (Figure 3.2 and 3.3). This suggested the synthesis of a new phase under the high-pressure and temperature conditions.

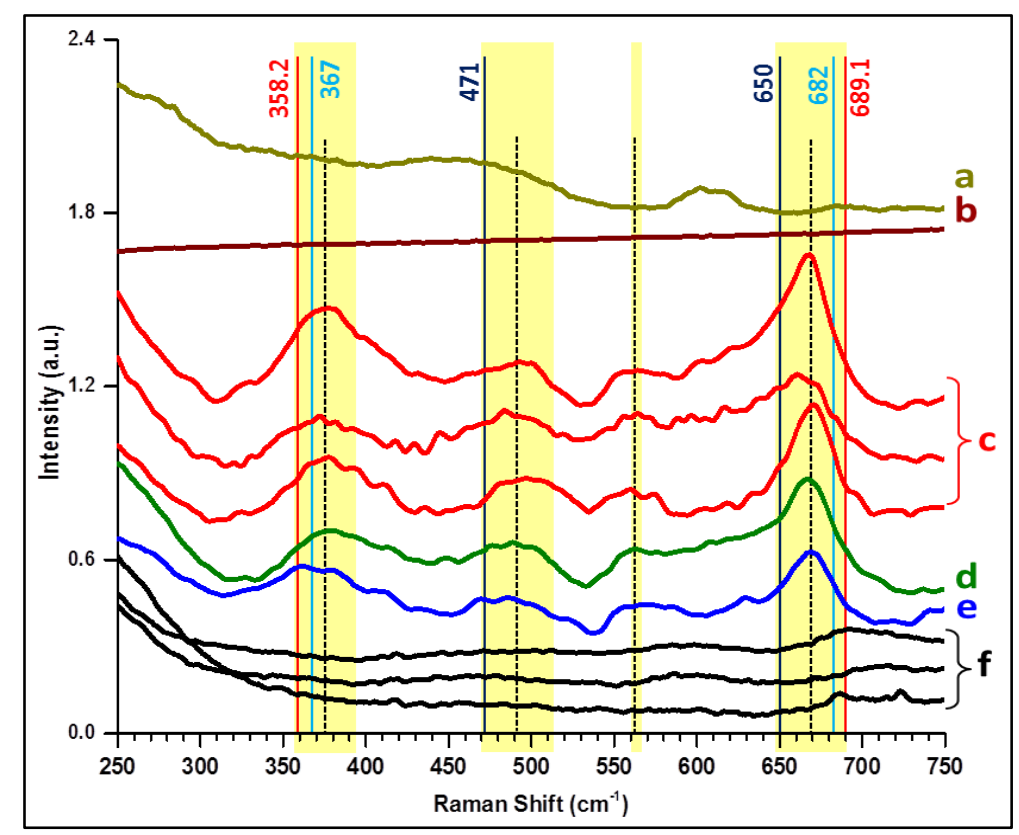

Figure $3.1 \mid$ Representative Raman spectra of various samples: (a) $\mathrm{Ag}_{2} \mathrm{O}\left(\mathrm{T}=600^{\circ} \mathrm{C}, \mathrm{P}=\right.$ $10 \mathrm{GPa})$, (b) $\mathrm{Ga}_{2} \mathrm{O}_{3}\left(\mathrm{~T}=600{ }^{\circ} \mathrm{C}, \mathrm{P}=10 \mathrm{GPa}\right)$, (c) Mixed powder of $\mathrm{Ag}_{2} \mathrm{O}+\mathrm{Ga}_{2} \mathrm{O}_{3}(1: 1$, 
$\left.\mathrm{T}=600{ }^{\circ} \mathrm{C}, \mathrm{P}=10 \mathrm{GPa}\right),(\mathrm{d})$ Mixed powder of $\mathrm{Ag}_{2} \mathrm{O}+\mathrm{Ga}_{2} \mathrm{O}_{3}\left(3: 1, \mathrm{~T}=600^{\circ} \mathrm{C}, \mathrm{P}=\right.$ $10 \mathrm{GPa}$ ), (e) Mixed powder of $\mathrm{Ag}_{2} \mathrm{O}+\mathrm{Ga}_{2} \mathrm{O}_{3}\left(1: 1, \mathrm{~T}=480{ }^{\circ} \mathrm{C}, \mathrm{P}=10 \mathrm{GPa}\right)$, (f) Mixed powder of $\mathrm{Ag}_{2} \mathrm{O}+\mathrm{Ga}_{2} \mathrm{O}_{3}\left(1: 2, \mathrm{~T}=600{ }^{\circ} \mathrm{C}, \mathrm{P}=\right.$ ambient $)$. Vertical red lines represent the location of our theoretically calculated Raman modes. Vertical light blue lines show the positions of Raman modes calculated by Kumar and Gupta [136]. Vertical dark blue lines show the location of Raman peaks measured by Nagarajan and Tomar [80].

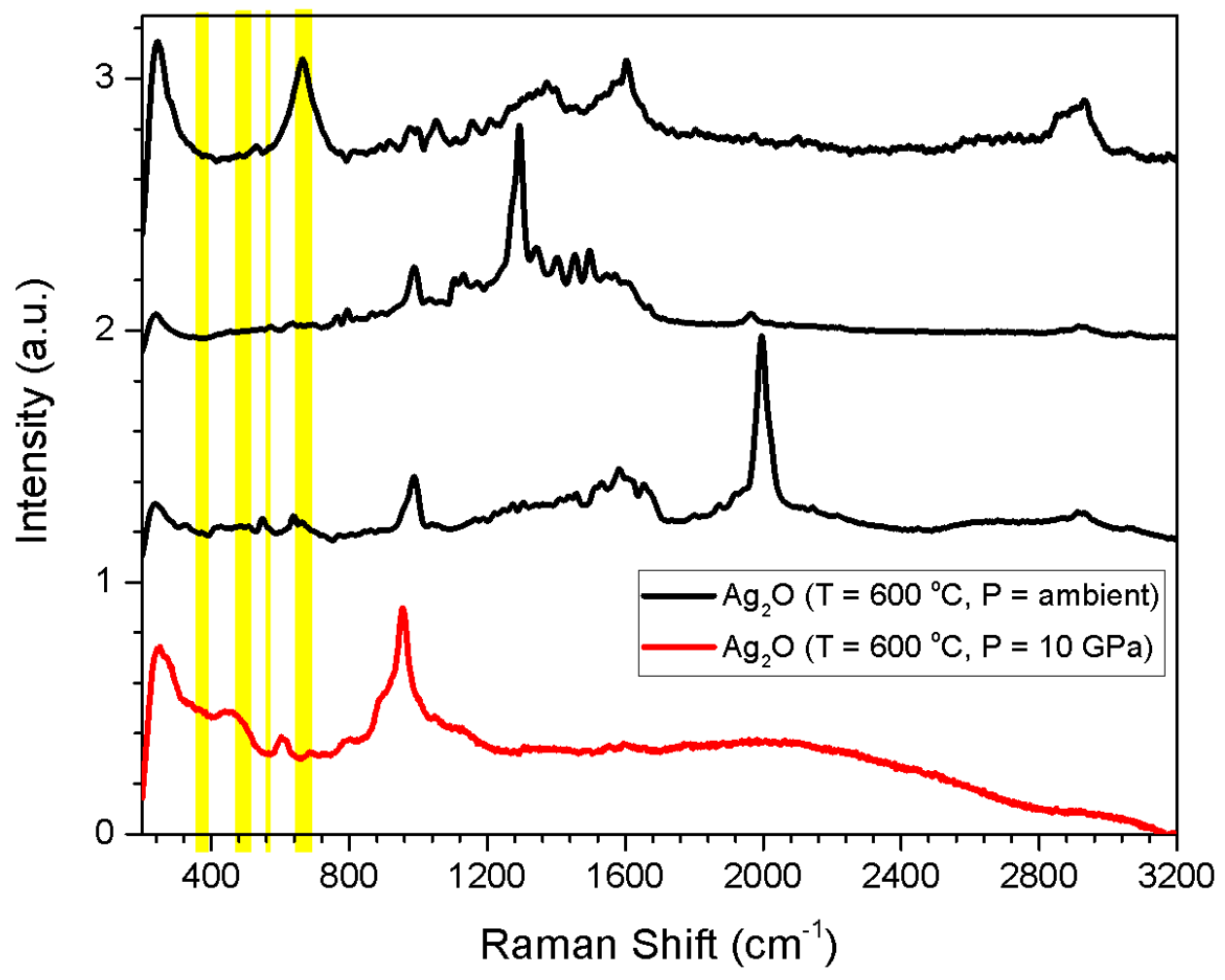

Figure 3.2 | Raman spectra of pure silver (I) oxide before (top three curves) and after (bottom curve) DAC experiment. Before applying high pressure, $\mathrm{Ag}_{2} \mathrm{O}$ is unstable under the illumination of $633 \mathrm{~nm}$ laser during Raman measurements. Hence, several shown spectra, measured from the same area at different times differ from each other (top three curves). However, after the high-pressure experiment, $\mathrm{Ag}_{2} \mathrm{O}$ is stable, and its spectrum 
(bottom curve) does not change under laser illumination. The spectral regions shaded in yellow indicate the location of four peaks characteristic of rhombohedral $\alpha-\mathrm{AgGaO}_{2}$.

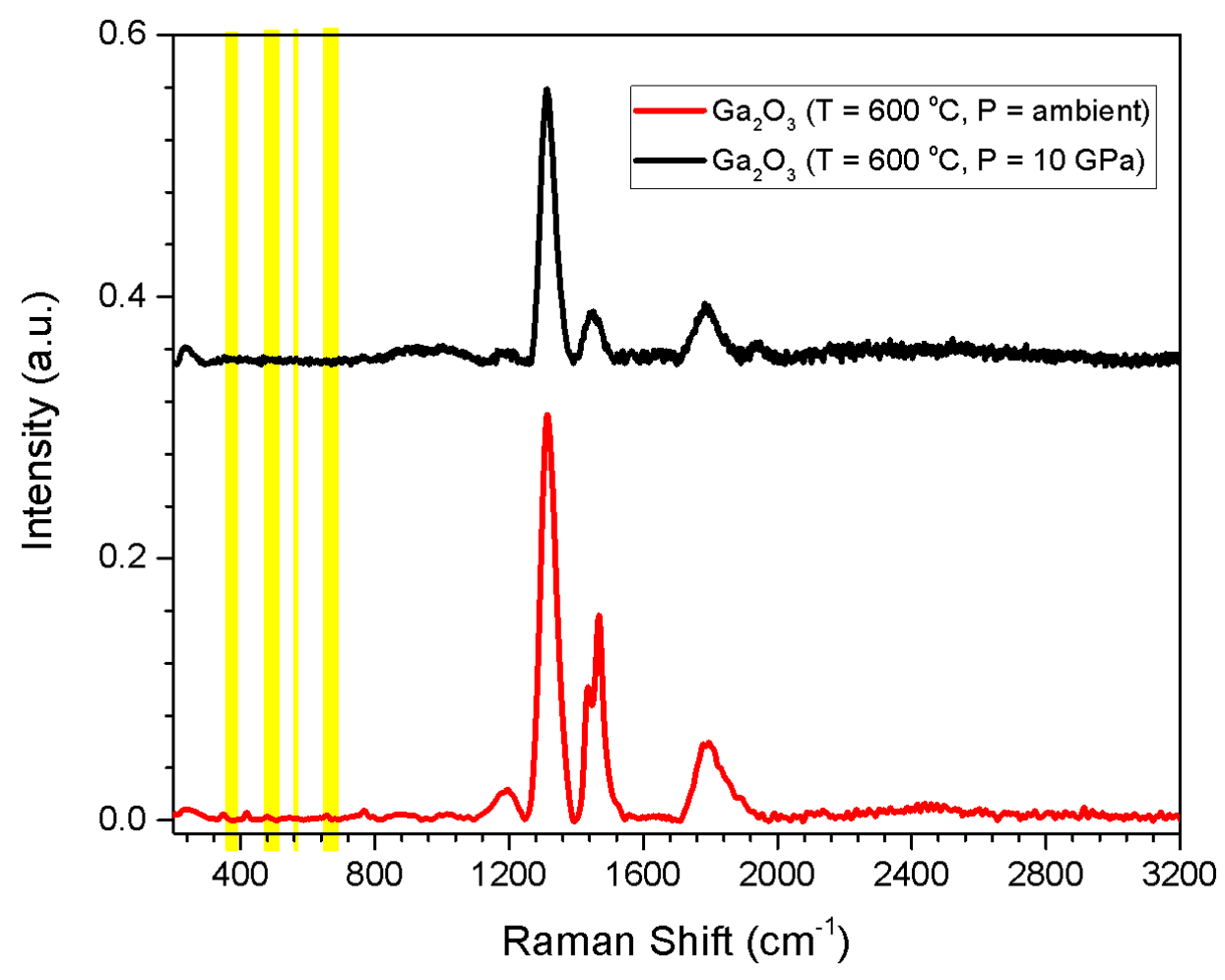

Figure 3.3 | Gallium (III) oxide before (top curve) and after (bottom curve) applying high pressure. The spectral regions shaded in yellow indicate the location of four peaks characteristic of rhombohedral $\alpha-\mathrm{AgGaO}_{2}$. 


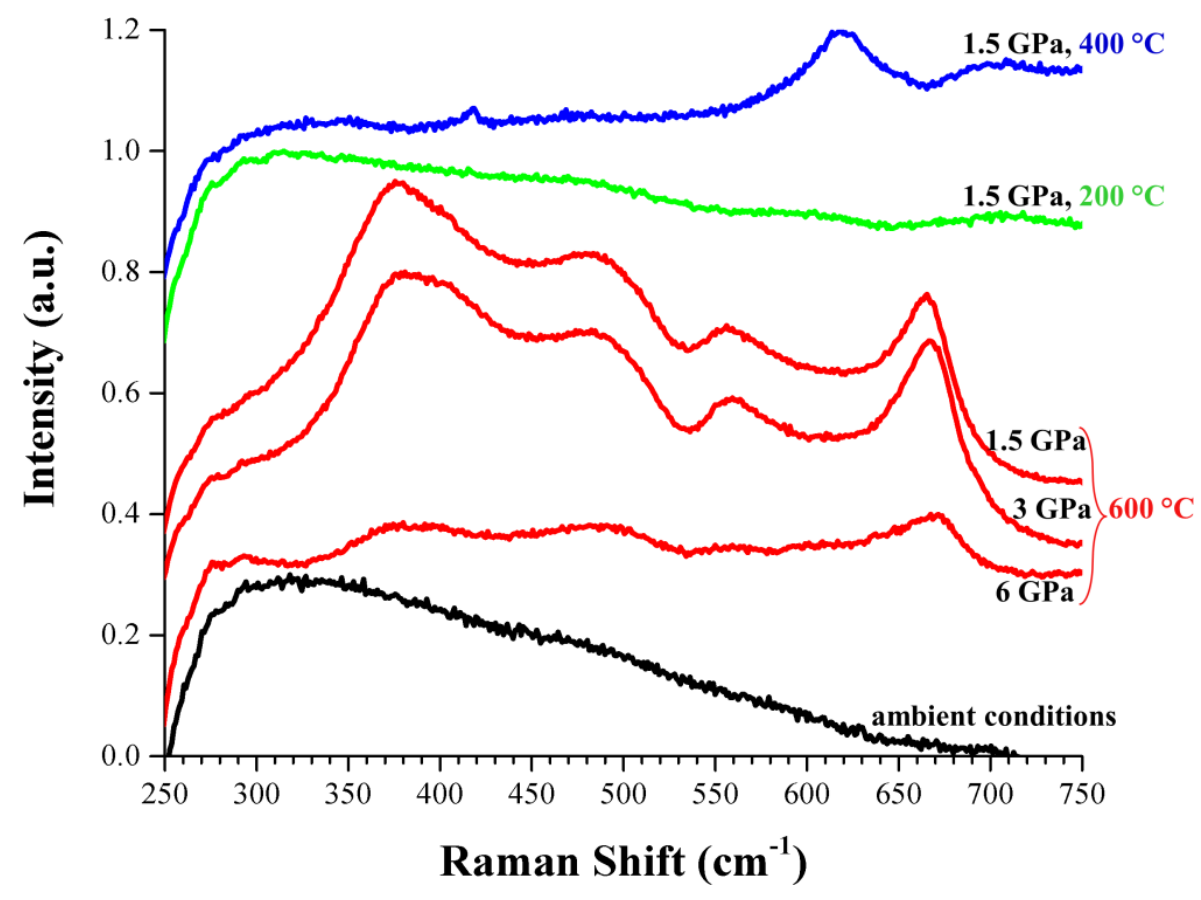

Figure 3.4 | Representative Raman spectra of a mixed powder of $\mathrm{Ag}_{2} \mathrm{O}+\mathrm{Ga}_{2} \mathrm{O}_{3}$ with a molar ratio of 1:1 in different pressure and temperature conditions. Characteristic Raman spectrum of rhombohedral $\alpha-\mathrm{AgGaO}_{2}$ is only present in red spectra. 


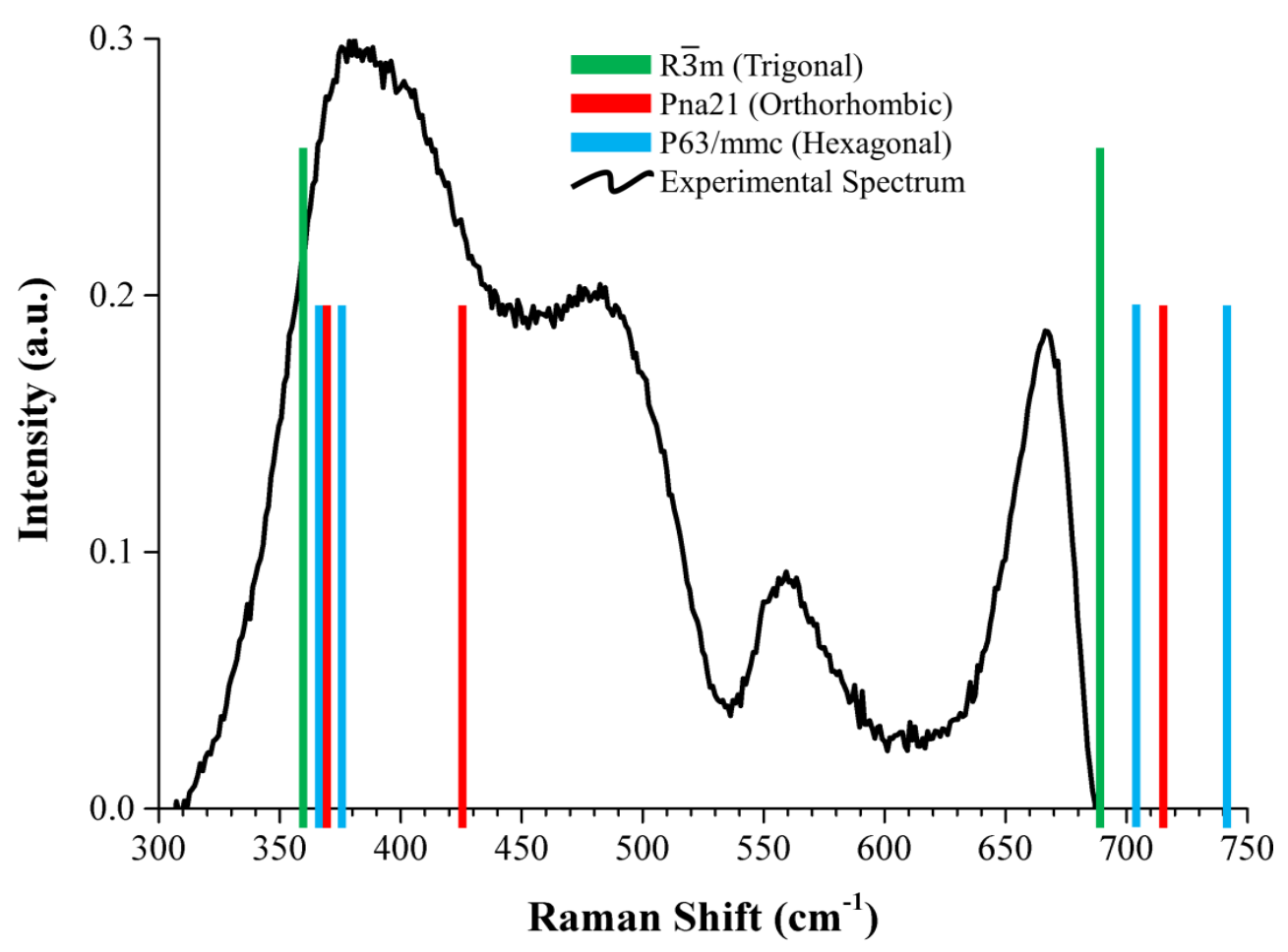

Figure 3.5 | The experimental Raman spectrum of DAC-synthesized $\mathrm{AgGaO}_{2}$ and calculated frequencies of Raman modes for the three known structures of $\mathrm{AgGaO}_{2}$. (There is a five percent uncertainty due to temperature effects.)

To study the structure and morphology of the new phase, we used electron microscopy. SEM results showed relatively uniform nanocrystals in scale of 100-200 nm (Figure 3.6 a). The nanocrystals exhibited surface faceting, which pointed to a highly crystalline structure. This was confirmed by TEM, which again revealed faceted nanocrystals in the same size range (Figure 3.6 b-e). EDX analysis (Figure 3.6) showed that the nanocrystals composed of $\mathrm{Ag}, \mathrm{Ga}$ and $\mathrm{O}$ with the atomic ratio of 1:1:2, respectively. This confirmed that $\mathrm{AgGaO}_{2}$ was synthesized during high-pressure annealing procedure. We also collected EDX elemental maps from the synthesized material using a $1 \mathrm{~nm}$ nanoprobe. The maps provided elemental distributions of $\mathrm{Ag}, \mathrm{Ga}$, and $\mathrm{O}$ that coincided with the 
shape of the crystallite (Figure $3.6 \mathrm{f}$ ). Therefore, the data strongly supports the following solid-state direct reaction:

$$
\mathrm{Ag}_{2} \mathrm{O}+\mathrm{Ga}_{2} \mathrm{O}_{3} \rightarrow 2 \mathrm{AgGaO}_{2}
$$

There are three known structures of $\mathrm{AgGaO}_{2}$ : rhombohedral $\alpha-\mathrm{AgGaO}_{2}$, hexagonal $\alpha-\mathrm{AgGaO}_{2}$, and orthorhombic $\beta-\mathrm{AgGaO}_{2}$ [137]. The most straightforward method to differentiate between them is X-ray diffraction (XRD). However, due to the minuscule amount of available material obtained from the DAC synthesis (c.a. $3 \mu \mathrm{g}$ in the case of our cell), XRD measurements would require a very intense X-ray source such as synchrotron radiation. Therefore, to identify which phase was formed, we conducted a detailed electron diffraction study instead. For this, electron diffraction ring patterns were acquired from different areas of the TEM grid containing groups of nanocrystals. Because the nanocrystals were very sparsely dispersed on the grid and the groups consisted of only few dozens of nanocrystals, the individual ring patterns were very spotty (Figure 3.9 a). Therefore, the analysis could be significantly hampered by the data quality. However, averaging all our data improved the ring pattern quality greatly (Fig. 3b). We also calculated the rotational average using DiffTools software [138]. The intensity profile was analyzed with Bruker DIFFRAC.SUITE ${ }^{\mathrm{TM}}$ software package. First, we considered all the possible crystallographic phases for the combination of gallium, Silver and oxygen. Then we looked through the ICDD PDF2 database to find the best match for the diffraction pattern. The lattice parameters of the best-matching structure, were tuned through the DIFFRAC.EVA software. To fit the the experimental pattern we used the rhombohedral delafossite crystal structure with the lattice parameters $a=2.99 \AA$ and $\mathrm{c}=18.43 \AA$ and the the $\mathrm{R} \overline{3} \mathrm{~m}$ space group. These parameters matched perfectly with 
the crystal structure of rhombohedral $\alpha-\mathrm{AgGaO}_{2}$ which belongs to the delafossite type compounds family with the general form of $\mathrm{A}^{\mathrm{I}} \mathrm{B}^{\mathrm{III}} \mathrm{O}_{2}$. The structure consists of alternating "A" layers of two-dimensional closed-packed " $\mathrm{A}$ " " ions in the dumbbell $\mathrm{O}-\mathrm{A}^{+}-\mathrm{O}$ coordination and slightly distorted edge-shared $\mathrm{BO}^{-6}$ octahedral layers perpendicular to the $\mathrm{c}$ axis $[81,82]$.

We also analyzed the electron diffraction pattern using the pattern matching method and FullProf software suite [139]. The crystal structure model was chosen based on the rhombohedral space group R $\overline{3} \mathrm{~m}$ (Figure $3.9 \mathrm{~d}$ ). The solid-line in Figure $3.9 \mathrm{~d}$ is obtained by calculating for the X-ray case. Thus, it cannot be directly compared with the experimental result. A similar study of the other two recognized $\mathrm{AgGaO}_{2}$ structures (with P63/mmc (194) and (c) Pna21 (33) space groups) showed a poor agreement (Figure 3.8). For a hexagonal structure, the refined lattice parameters are $\mathrm{a}=2.996(10) \AA$ and $\mathrm{c}=$ $18.986(124) \AA$. These values are in great agreement with the lattice parameters associated to rhombohedral $\alpha-\mathrm{AgGaO}_{2}$. 


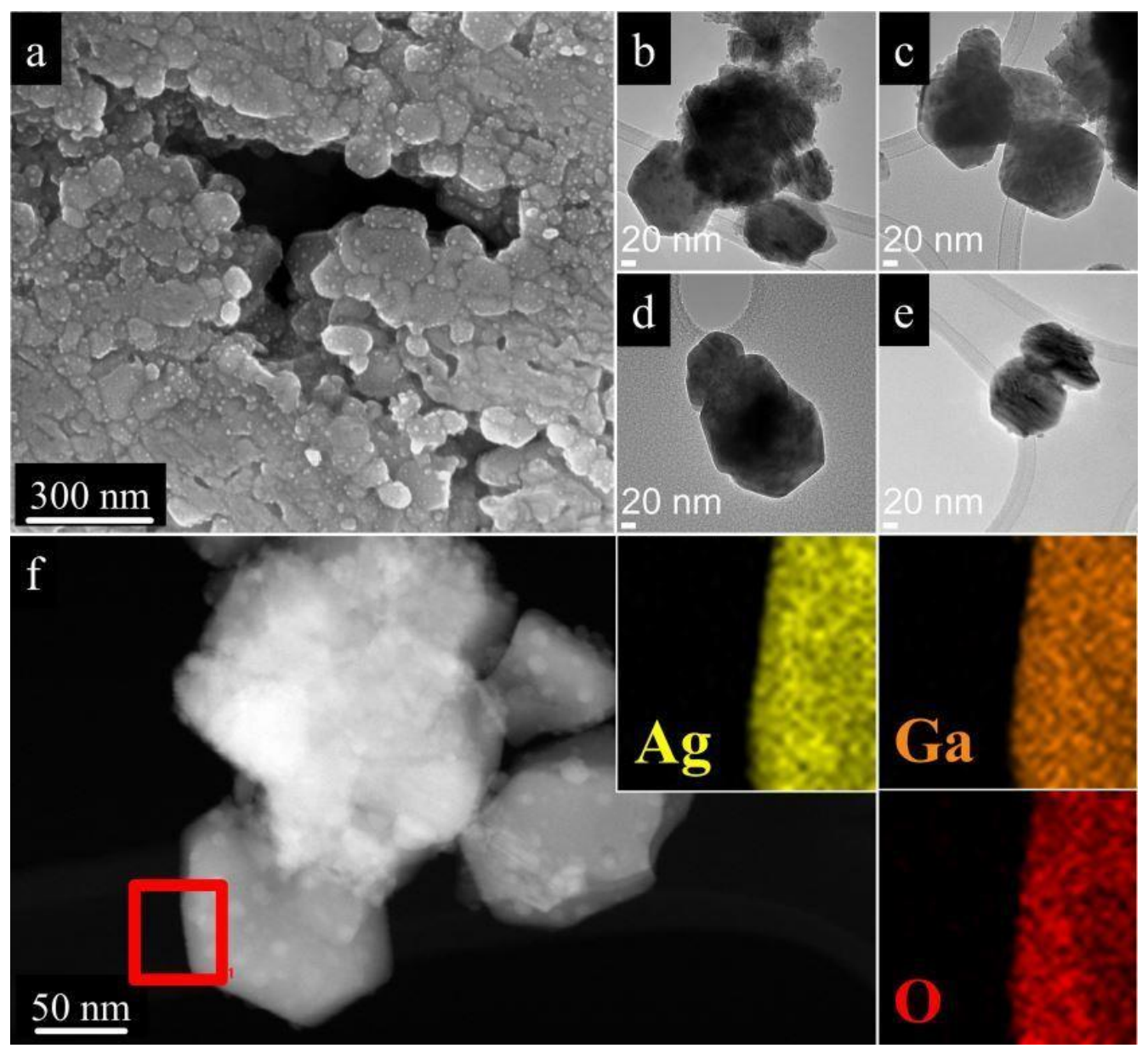

Figure 3.6 | Electron microscopy characterization of $\mathrm{AgGaO}_{2}$ nanocrystals synthesized during high-temperature DAC experiments: (a) SEM and (b-e) TEM images showing the size and morphology of the nanocrystals. (f) EDX-based elemental mapping was confirming the uniformity and perfect overlap of elemental distributions of $\mathrm{Ag}, \mathrm{Ga}$, and $\mathrm{O}$ (insets). 


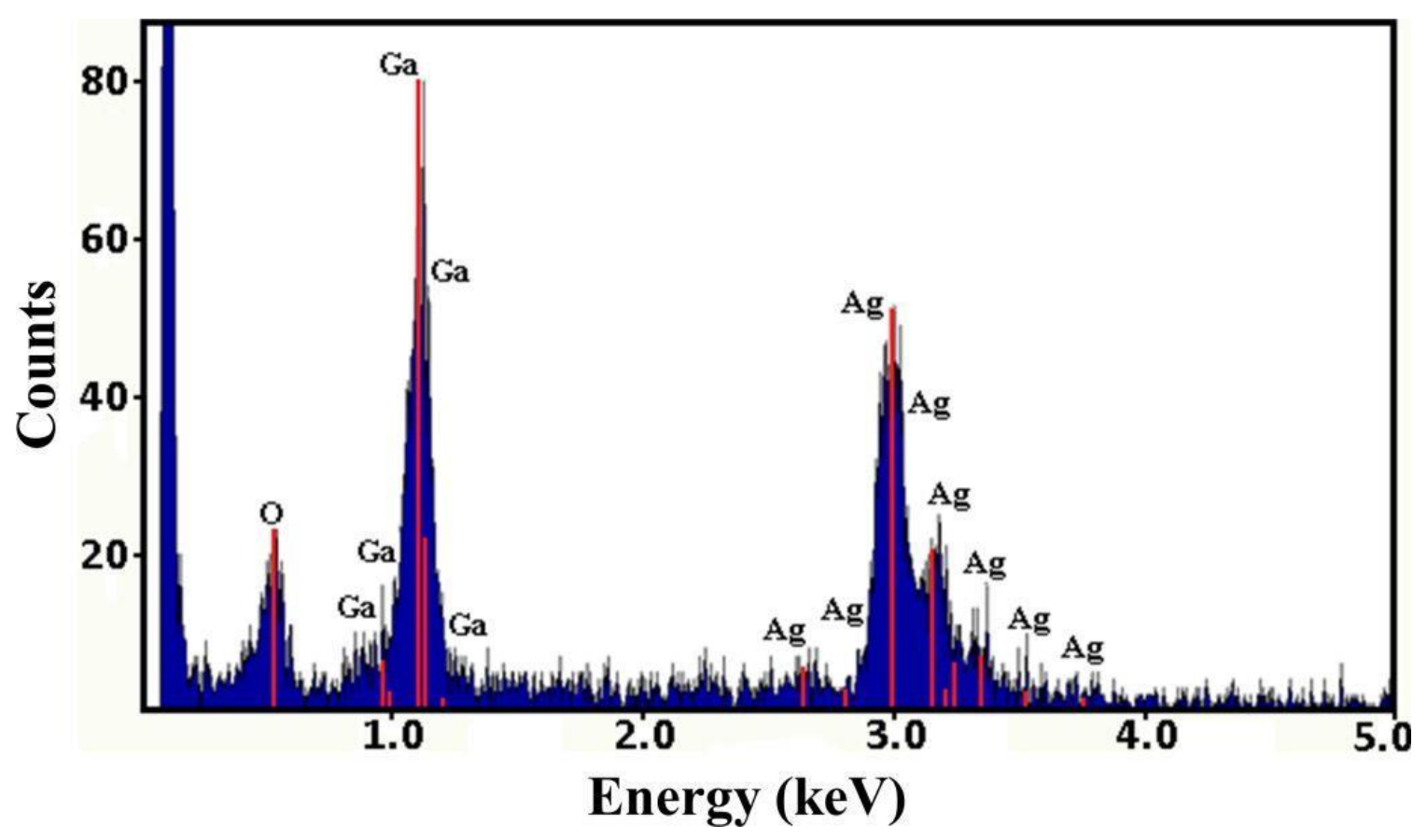

Figure 3.7 $\mid$ An EDX spectrum from a typical $\alpha-\mathrm{AgGaO}_{2}$ crystallite synthesized at high pressure.

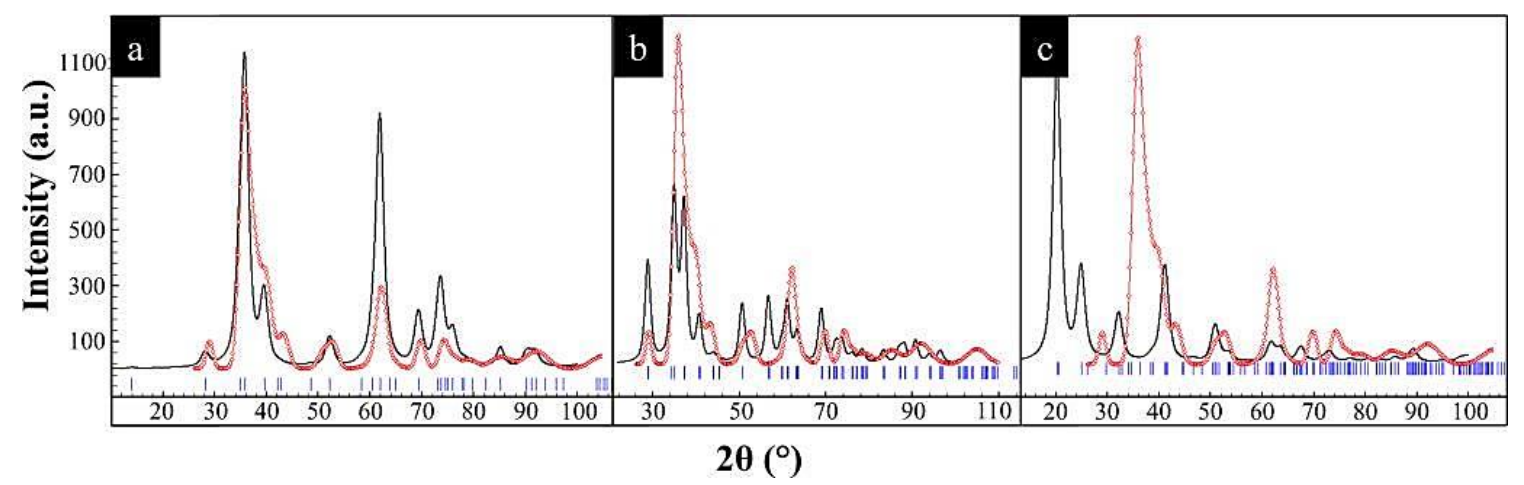

Figure 3.8 Results of the FullProf-based pattern matching analysis (black curves) of the radial intensity distribution (red curve) of the averaged SAED pattern of the DACsynthesized $\mathrm{AgGaO}_{2}$ for crystal structure models of $\mathrm{AgGaO}_{2}$ with (a) $\mathrm{R} \overline{\mathbf{3}} \mathrm{m}$ (166), (b) P63/mmc (194) and (c) Pna21 (33) space groups. It can be seen that the best fit was obtained for the $\mathrm{R} \overline{\mathbf{3}} \mathrm{m}$ (166) space group. 
The crystal structure was futher confirmed using single-crystal electron diffraction from individual $\mathrm{AgGaO}_{2}$ crystallites. Two examples of typical single crystal SAED patterns and associated HRTEM images are demonstrated in Figure 3.10. The d-spacing values obtained from these methods were about $2.49 \AA$ and $2.26 \AA$ respectively which matched well with the d-values of the most intense reflections of the $\alpha-\mathrm{AgGaO}_{2}$ with the rhombohedral R $\overline{3} \mathrm{~m}$ crystal structure. 

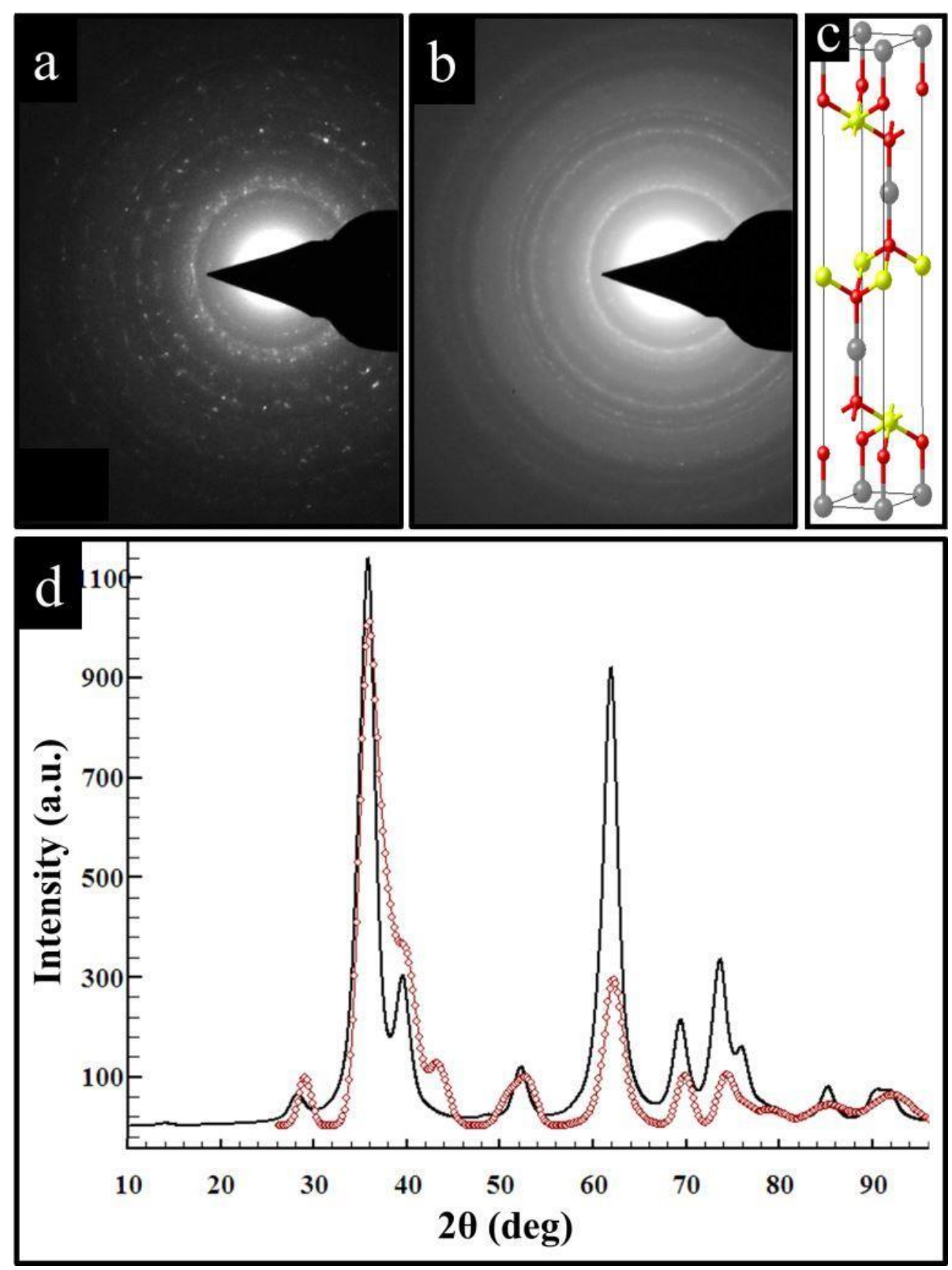

Figure 3.9 | (a) A SAED ring pattern acquired from a typical cluster of a few $\mathrm{AgGaO}_{2}$ crystallites. (b) The SAED pattern obtained after averaging the data from about 40 separate clusters. (c) The optimized crystal structure of $\alpha-\mathrm{AgGaO}_{2}$ using our DFT method (see text). (d) Experimental intensity profile obtained after rotational averaging of the pattern shown in $\mathrm{b}$ (red curve) and diffraction pattern calculated using the pattern matching mode of the FullProf software suite (black curve). 


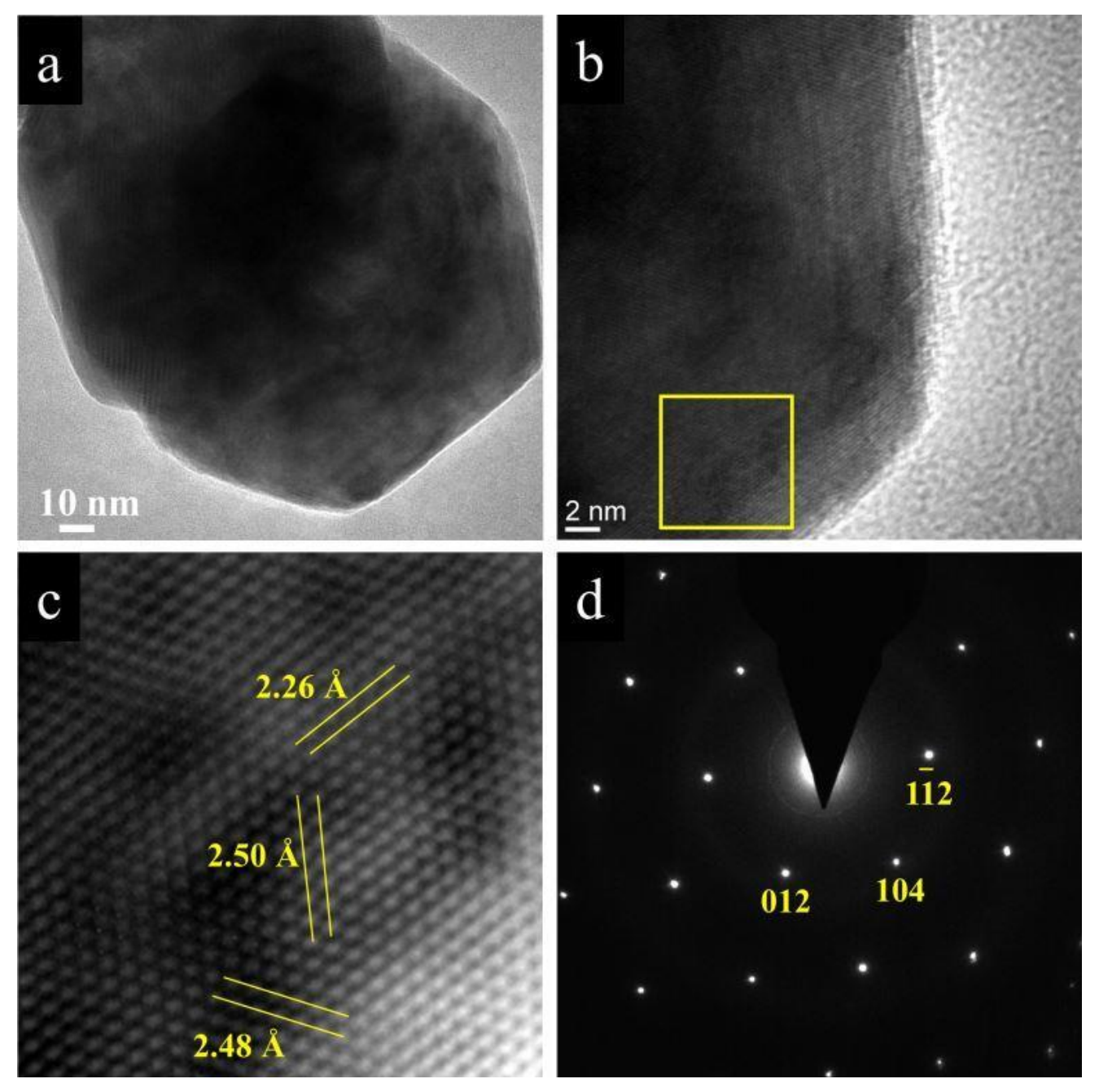

Figure 3.10 | TEM-based single crystal study of individual $\alpha-\mathrm{AgGaO}_{2}$ crystallite: (a) and (b) low-magnification and high-resolution TEM images; (c) Filtered HRTEM image of the area marked using yellow frame in b; (d) corresponding [42 $\overline{\mathbf{1}}$ ] zone axis SAED pattern measured from this crystallite. 
Table 3.1 | Crystallographic Parameters Obtained for the DFT-Optimized $\alpha-\mathrm{AgGaO}_{2}$.

\begin{tabular}{|l|l|}
\hline Cell lattice parameter a & $2.87428(\AA)$ \\
\hline Cell lattice parameter b & $2.87428(\AA)$ \\
\hline Cell lattice parameter c & $18.28372(\AA)$ \\
\hline Cell angle alpha & $90.00\left(^{\circ}\right)$ \\
\hline Cell angle beta & $90.00\left(^{\circ}\right)$ \\
\hline Cell angle gamma & $120.00\left(^{\circ}\right)$ \\
\hline Cell volume & $130.81\left(\AA^{3}\right)$ \\
\hline Cell formula units $Z$ & 3 \\
\hline Space group & $\mathrm{R}^{\circ} \mathrm{m}$ \\
\hline Volume & $130.814\left(\AA^{3}\right)$ \\
\hline Moiety formula & $\mathrm{AgGaO}$ \\
\hline $\mathrm{D}_{\mathrm{x}, \mathrm{g}} \mathrm{cm}^{-3}$ & 7.982 \\
\hline $\mathrm{Z}$ & 3 \\
\hline Mu (mm & \\
\hline F000 & 26.201 \\
\hline F000' & 280.11 \\
\hline
\end{tabular}

Theoretical analysis of vibrational modes was performed to explain the Raman peaks ${ }^{2}$.

For the theoretical study of rhombohedral $\mathrm{AgGaO}_{2}$ systems of $\mathrm{R} \overline{3} \mathrm{~m}$ symmetry, we used first-principles density functional theory (DFT) in the generalized gradient approximation (GGA) and the Perdew-Burke-Ernzerhof (PBE) [27] form augmented by including

\footnotetext{
${ }^{2}$ Performed by Dr. Madhu Menon (Center for Computational Sciences at the University of Kentucky)
} 
Hubbard- $U$ corrections (GGA $+U$ formalism) [140] based on Dudarev's approach [141] as implemented in the Vienna ab initio simulation package (VASP) [142]. The $U$ values used for $\mathrm{Ga}$ and $\mathrm{O}$ were $U_{\mathrm{d}, \mathrm{Ga}}=6.5 \mathrm{eV}$ and $U_{\mathrm{d}, \mathrm{O}}=14.0 \mathrm{eV}$, respectively. The projected augmented wave (PAW) potential [141] is used to describe the core electrons. After testing for convergence, we settled for a $5 \times 5 \times 5 \Gamma$-centered pack for $\mathbf{k}$-vectors sampling. We found that the adequate kinetic energy cutoff for reaching a total energy convergence of $1 \mathrm{meV}$, was $550 \mathrm{eV}$. To accelerate the electronic convergence, a Gaussian smearing of $0.05 \mathrm{eV}$ was used. It was acceptable to optimize the atomic positions to proceed without symmetry constraints till the force experienced by each atom is less than $5 \mathrm{meV} / \AA$. The improved structure led to the lattice parameters listed in Table 3.1. For calculating the phonon modes of rhombohedral $\alpha-\mathrm{AgGaO}_{2}$, the VASP code was used. The calculated vibrational modes are at 79.88, 9.04, 295.54, 358.51, 549.01 (infra-red active modes), 358.21 and $689.14 \mathrm{~cm}^{-1}$ (Raman-active modes).

We also calculated the vibrational modes in hexagonal orthorhombic $\beta-\mathrm{AgGaO}_{2}$ and $\alpha-$ $\mathrm{AgGaO}_{2}$ structures (Figure 3.5). The result for the rhombohedral $\alpha-\mathrm{AgGaO}_{2}$ structure was greatly matching the Raman data. In particular, the two strongest Raman peaks matched with the calculated Raman vibrational modes (Table 3.2). The calculated vibrational modes for the other structures did not match with the experimental data. This further confirms the identified phase. While the ab initio calculations did not predict the two weaker peaks in our Raman spectra, they could be noted in the previously reported data for rhombohedral $\alpha-\mathrm{AgGaO}_{2}$ made by other techniques [80].

To understand the other two Raman modes in our data, we examined all the potential vibrational and stretching or bending modes associated with oxygen and silver bonds in 
the $\mathrm{AgGaO}_{2}$ structure. The two Raman peaks are in excellent agreement with the stretching/bending modes of $\mathrm{Ag}-\mathrm{O}$ bond [143]. Due to the distortions of atom clusters through the recovery from high-temperature and high-pressure, the peaks are stronger in our samples. This could be the reason for the decreased symmetry, enhancement of weak Raman modes and relaxation of selection rules.

Our experimental and theoretical data is consistent with the DFT calculations reported by Kumar and Gupta [136]. They compared their theoretical results with experimental Raman data published by Nagarajan and Tomar [80]. They suggested potential misassignment of $\mathrm{E}_{\mathrm{g}}$ mode near $471 \mathrm{~cm}^{-1}$. However, according to our results the stretching/bending mode of $\mathrm{Ag}-\mathrm{O}$ bond is responsible for appearing this peak and it does related to $\mathrm{E}_{\mathrm{g}}$ mode.

Employing our DFT method, we were able to estimate the band gap energy of $\alpha$ $\mathrm{AgGaO}_{2}$. Previously reported theoretical studies for this phase have only used the standard DFT. This would underestimate the band gap energy. Subsequently, the band gap values are not mentioned in all the previous reports except the one by Maruyama et al. [77]. Even the energy gap estimated by Maruyama et al. is only $0.73 \mathrm{eV}$. In contrast, our calculations resulted in a band gap of $1.83 \mathrm{eV}$ for this compound. This is much closer to the reported experimental value of $2.38 \mathrm{eV}[77,137,144]$. 


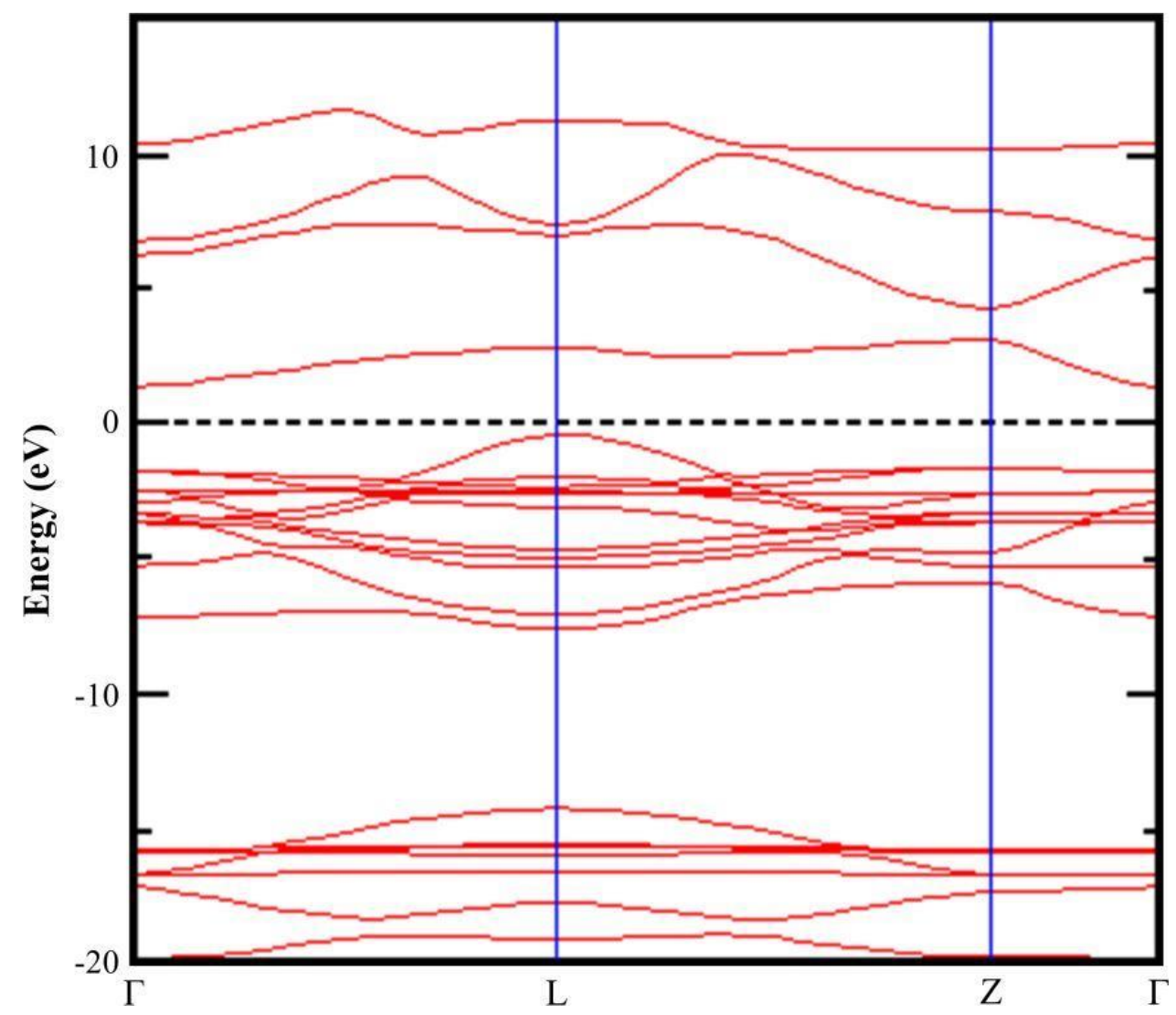

Figure 3.11 | The calculated electronic energy band structure of $\alpha-\mathrm{AgGaO}_{2}$

Table 3.2 | Experimental and calculated Raman modes.

\begin{tabular}{|c|c|c|c|c|}
\hline \multirow{2}{*}{$\begin{array}{c}\text { Raman Mode } \\
\left(\mathrm{cm}^{-1}\right)\end{array}$} & \multicolumn{2}{|c|}{ Theor. Calculation } & \multicolumn{2}{c|}{ Exp. Result } \\
\cline { 2 - 5 } & $\begin{array}{c}\text { Kumar \& } \\
\text { Gupta [136] }\end{array}$ & This work & $\begin{array}{c}\text { Nagarajan \& } \\
\text { Tomar [80] }\end{array}$ & This work \\
\hline $\mathrm{E}_{\mathrm{g}}$ & 367 & 358.2 & - & 375 \\
\hline $\mathrm{Ag}-\mathrm{O}$ & - & - & 471 & 485 \\
\hline $\mathrm{Ag}-\mathrm{O}$ & - & - & - & 560 \\
\hline $\mathrm{A}_{1 \mathrm{~g}}$ & 682 & 689.1 & 650 & 670 \\
\hline
\end{tabular}




\subsection{Three-Dimensional (3D) Graphene Architecture}

We developed a nickel (Ni) foam assisted pyrolysis method as a compelling approach to achieve larger grains for enhanced growth quality and to control the morphology of 3D graphene networks. Ni foam with large grain boundaries is a suitable template for 3D graphene growth due to its surface smoothness, porosity, and grain size. However, it is well known that controlling the number of graphene layers is very challenging via nickel assisted growth methods.

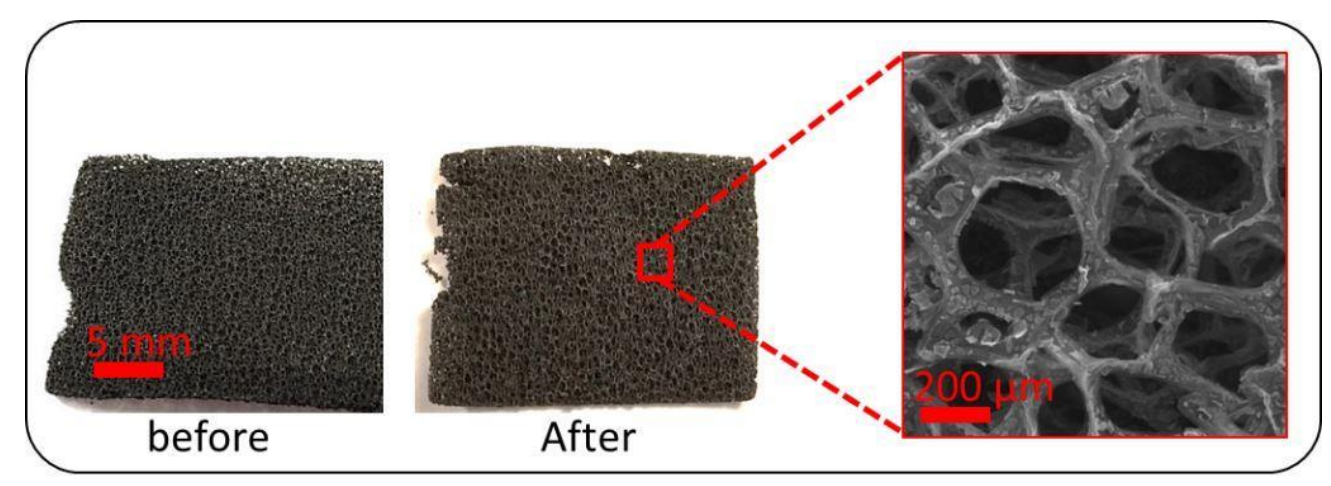

Figure 3.12 | A SEM Image of 3D Graphene After Etching Ni Foam with HCl 5M (Right) and Photographs of 3D graphene Samples Before and After Etching Ni foam (right).

Raman spectroscopy, both transmission and scanning types of electron microscopy, electron energy loss spectroscopy (EELS), and X-ray photoelectron spectroscopy (XPS) were utilized for a thorough characterization of the 3D graphene samples. 


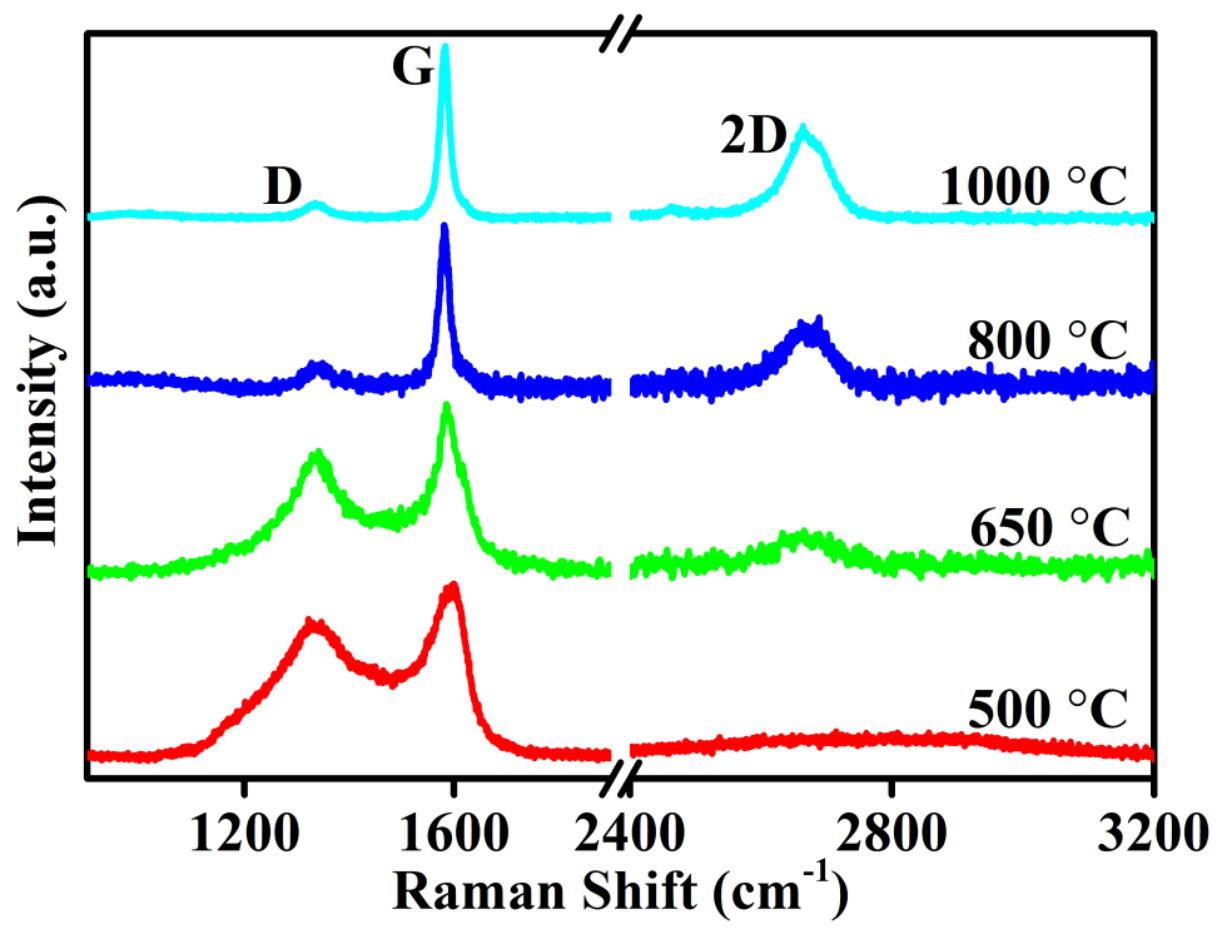

Figure 3.13 | A representative averages of obtained Raman spectra from 3D graphene foam grown at different temperatures.

Raman spectroscopy measurements were conducted for all 3D graphene samples prepared at various temperatures $\left(500,650,800\right.$, and $\left.1000{ }^{\circ} \mathrm{C}\right)$ to analyze the structural quality. A representative set of obtained spectra is presented in Figure 3.13. It can be seen that all samples have three prominent Raman peaks at $\sim 1,350 \mathrm{~cm}^{-1}$ ( $\mathrm{D}$ band), 1,580 $\mathrm{cm}^{-1}$ ( $\mathrm{G}$ band), and $\sim 2,700 \mathrm{~cm}^{-1}$ (2D band) which are chatracteristic to graphene Ramanactive vibrational modes. It is well established that the D band, $\mathrm{G}$ band, and 2D band originate from defects, the in-plane vibration of $\mathrm{sp}^{2}$-hybridized carbon atoms, and second-order double resonant Raman, respectively [145-147]. The defects can be caused by vacancies, dislocations, edges, and cracks in graphene crystal structure. It is expected for 3D graphene samples to have more defect than a flat sheet of graphene and consequently more intense $\mathrm{D}$ bands. This is due to the substrate ( $\mathrm{Ni}$ foam) surface with 
many curvatures and edges, in contrast to conventional foil substrates. It is well known that Raman spectroscopy can reveal significant information about quality and number of graphene layers. Based on the Raman data of our samples (Figure 3.13), the intensity ratio of $\mathrm{D}$ and $\mathrm{G}$ bands $\left(\mathrm{I}_{\mathrm{D}} / \mathrm{I}_{\mathrm{G}}\right)$ decreased by increasing the temperature and reached to the lowest value at $1000{ }^{\circ} \mathrm{C}$. This trend is an indication of higher crystallinity structure with lower defect concentration. Moreover, the ratio of the intensity of $2 \mathrm{D}$ bands and $\mathrm{G}$ bands $\left(\mathrm{I}_{2 \mathrm{D}} / \mathrm{I}_{\mathrm{G}}\right)$ rose significantly by increasing the temperature and reached its highest values at $1000{ }^{\circ} \mathrm{C}$. Trends in both intensity ratios indicated that the 3D graphene grown at a higher temperature has better overall structural quality, and less number of layers. The highest $3 \mathrm{D}$ graphene was achieves at around $1000{ }^{\circ} \mathrm{C}$. From the Raman analysis of the graphene foam samples, the $\mathrm{I}_{2 \mathrm{D}} / \mathrm{I}_{\mathrm{G}}$ ratio showed a characteristic of few-layered graphene coatings. This was also verified by high-resolution TEM image of the graphene coating. In addition, the low-intensity Raman D bands $\left(\sim 1350 \mathrm{~cm}^{-1}\right)$ of samples grown at $1000{ }^{\circ} \mathrm{C}$ in Figure 3.13 revealed a low defect density in the $3 \mathrm{D}$ graphene structure. 


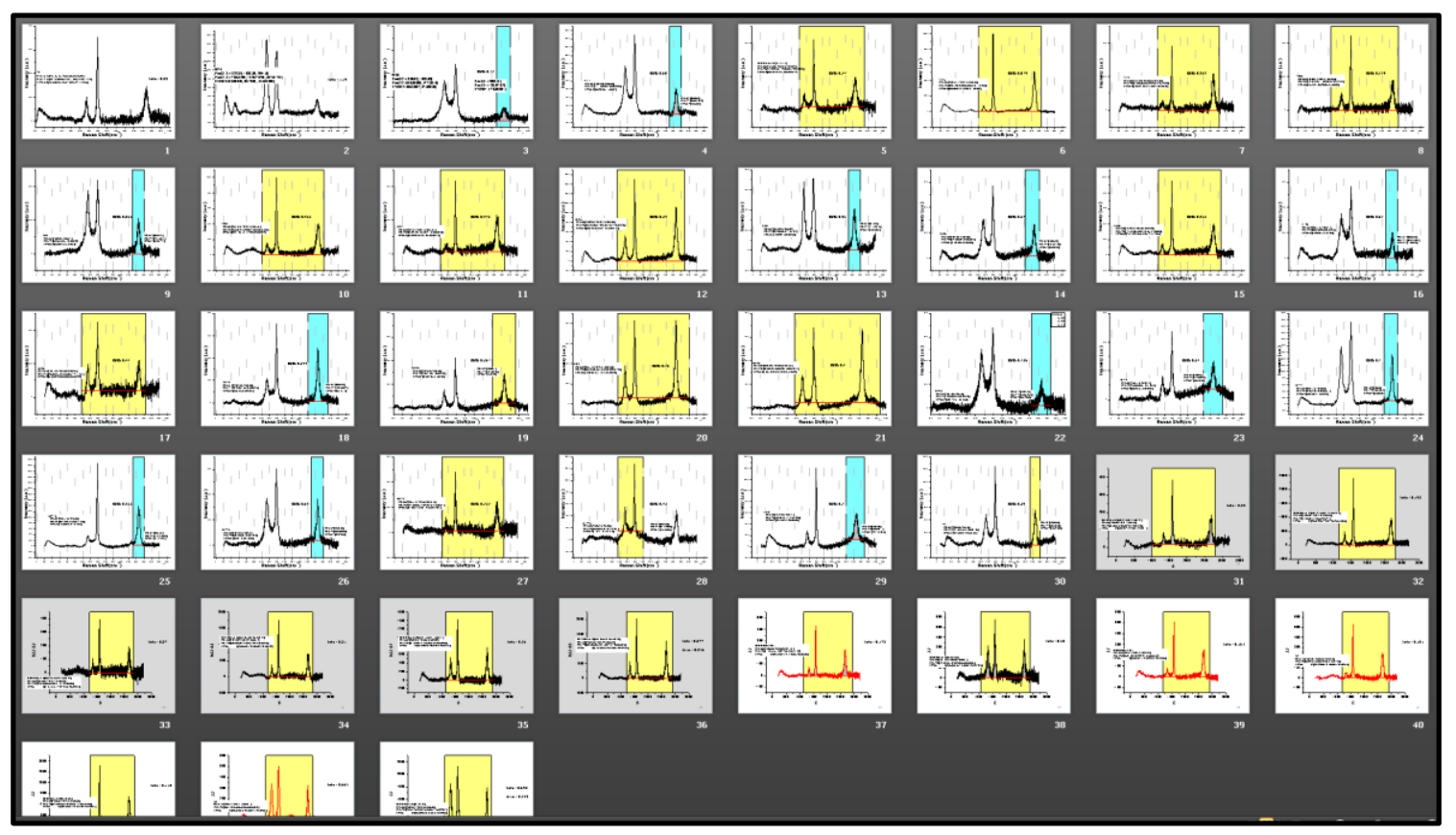

Figure 3.14 | Deconvolution of 3D graphene Raman peaks with a Lorentzian function to find FWHM, positions, and area under the peaks for statistical analysis. 


\begin{tabular}{|c|c|c|c|c|c|c|c|c|c|c|c|c|c|c|c|c|c|}
\hline 4 & A & C & D & E & $\mathrm{F}$ & G & $\mathrm{H}$ & 1 & 1 & K & L & M & N & 0 & $\mathrm{P}$ & Q & $\mathrm{R}$ \\
\hline 1 & $\mathrm{~T}$ & & $X_{D}$ & $\mathrm{X}_{\mathrm{G}}$ & $\mathrm{X}_{2 \mathrm{D}}$ & $\mathrm{I}_{\mathrm{D}}$ & $\mathrm{I}_{\mathrm{G}}$ & $\mathrm{I}_{2 \mathrm{D}}$ & $\mathrm{FWHM}_{\mathrm{D}}$ & $\mathrm{FWHM}_{\mathrm{G}}$ & $\mathrm{FWHM}_{2 \mathrm{D}}$ & $\mathrm{I}_{\mathrm{D}} / \mathrm{I}_{\mathrm{G}}$ & $\mathrm{I}_{2 \mathrm{D}} / \mathrm{I}_{\mathrm{G}}$ & $\mathrm{I}_{2 \mathrm{D}} / \mathrm{I}_{\mathrm{D}}$ & $\mathrm{W}_{\mathrm{D}} / \mathrm{W}_{\mathrm{G}}$ & $\mathrm{W}_{2 \mathrm{D}} / \mathrm{W}_{\mathrm{G}}$ & $\mathrm{W}_{2 \mathrm{D}} / \mathrm{W}_{\mathrm{D}}$ \\
\hline 2 & 650 & 624 & 1322.39 & 1588.36 & 2641.90 & 1708.32 & 1425.74 & 337.01 & 68.05 & 68.70 & 104.54 & 1.19 & 0.24 & 0.20 & 0.99 & 1.52 & 1.54 \\
\hline 3 & 650 & 1038 & 1339.08 & 1583.03 & 2663.81 & 345.65 & 477.80 & 120.97 & 66.08 & 37.95 & 176.96 & 0.72 & 0.25 & 0.35 & 1.74 & 4.66 & 2.68 \\
\hline 4 & 650 & 1864 & 1329.81 & 1582.14 & 2660.17 & 373.84 & 437.03 & 212.48 & 58.55 & 54.75 & 80.30 & 0.86 & 0.49 & 0.57 & 1.07 & 1.47 & 1.37 \\
\hline 5 & 650 & 2281 & 1327.96 & 1568.79 & 2647.02 & 679.25 & 714.84 & 414.09 & 49.79 & 59.86 & 78.25 & 0.95 & 0.58 & 0.61 & 0.83 & 1.31 & 1.57 \\
\hline 6 & 650 & 30108 & 1324.25 & 1584.80 & 2667.46 & 498.82 & 677.30 & 255.01 & 69.04 & 48.04 & 85.49 & 0.74 & 0.38 & 0.51 & 1.44 & 1.78 & 1.24 \\
\hline 7 & 800 & 313 & 1346.48 & 1580.36 & 2676.92 & 201.75 & 702.44 & 290.41 & 57.50 & 26.01 & 91.42 & 0.28 & 0.41 & 1.44 & 2.21 & 3.51 & 1.59 \\
\hline 8 & 800 & 729 & 1340.93 & 1582.14 & 2672.55 & 281.22 & 1403.01 & 596.68 & 60.48 & 25.96 & 101.42 & 0.20 & 0.43 & 2.12 & 2.33 & 3.91 & 1.68 \\
\hline 9 & 800 & 1141 & 1332.59 & 1580.36 & 2663.81 & 1144.53 & 1889.77 & 635.31 & 62.96 & 44.65 & 77.65 & 0.60 & 0.34 & 0.56 & 1.41 & 1.74 & 1.23 \\
\hline 10 & 800 & 1142 & 1345.55 & 1581.25 & 2676.92 & 101.97 & 419.75 & 198.13 & 52.25 & 26.42 & 96.51 & 0.24 & 0.47 & 1.94 & 1.98 & 3.65 & 1.85 \\
\hline 11 & 800 & 1556 & 1329.81 & 1579.47 & 2670.37 & 101.94 & 443.96 & 181.21 & 78.45 & 25.96 & 84.69 & 0.23 & 0.41 & 1.78 & 3.02 & 3.26 & 1.08 \\
\hline 12 & 800 & 1968 & 1324.25 & 1579.47 & 2664.54 & 155.62 & 999.31 & 398.64 & 90.14 & 24.11 & 98.38 & 0.16 & 0.40 & 2.56 & 3.74 & 4.08 & 1.09 \\
\hline 13 & 800 & 2285 & 1331.67 & 1581.25 & 2666.73 & 311.23 & 575.66 & 264.61 & 74.80 & 37.86 & 82.62 & 0.54 & 0.46 & 0.85 & 1.98 & 2.18 & 1.10 \\
\hline 14 & 800 & 2386 & 1349.25 & 1580.36 & 2676.92 & 147.40 & 959.47 & 401.30 & 71.74 & 25.54 & 110.41 & 0.15 & 0.42 & 2.72 & 2.81 & 4.32 & 1.54 \\
\hline 15 & 800 & 2798 & 1324.25 & 1577.69 & 2669.64 & 98.25 & 223.08 & 111.44 & 54.63 & 30.33 & 87.36 & 0.44 & 0.50 & 1.13 & 1.80 & 2.88 & 1.60 \\
\hline 16 & 800 & 31110 & 1334.45 & 1579.47 & 2669.64 & 162.38 & 472.49 & 271.66 & 57.95 & 29.77 & 83.76 & 0.34 & 0.57 & 1.67 & 1.95 & 2.81 & 1.45 \\
\hline 17 & 1000 & 1244 & 1336.30 & 1584.80 & 2662.36 & 494.57 & 1253.77 & 552.67 & 61.85 & 28.68 & 75.01 & 0.39 & 0.44 & 1.12 & 2.16 & 2.62 & 1.21 \\
\hline 18 & 1000 & 1245 & 1330.74 & 1579.47 & 2661.63 & 790.49 & 9986.95 & 5144.01 & 50.82 & 21.09 & 74.57 & 0.08 & 0.52 & 6.51 & 2.41 & 3.54 & 1.47 \\
\hline 19 & 1000 & 1247 & 1340.93 & 1578.58 & 2672.55 & 61.86 & 381.93 & 218.49 & 105.19 & 21.46 & 86.16 & 0.16 & 0.57 & 3.53 & 4.90 & 4.01 & 0.82 \\
\hline 20 & 1000 & 2071 & 1313.10 & 1579.47 & 2664.54 & 62.97 & 432.27 & 230.53 & 60.39 & 20.84 & 88.83 & 0.15 & 0.53 & 3.66 & 2.90 & 4.26 & 1.47 \\
\hline 21 & 1000 & 2075 & 1329.81 & 1579.47 & 2662.36 & 531.73 & 1690.06 & 1128.90 & 68.21 & 27.91 & 69.02 & 0.31 & 0.67 & 2.12 & 2.44 & 2.47 & 1.01 \\
\hline 22 & 1000 & 2488 & 1334.45 & 1582.14 & 2666.73 & 356.05 & 567.31 & 202.71 & 78.98 & 34.00 & 94.08 & 0.62 & 0.36 & 0.57 & 2.32 & 2.77 & 1.19 \\
\hline 23 & 1000 & 28102 & 1331.67 & 1581.25 & 2663.09 & 219.65 & 705.86 & 490.38 & 58.84 & 25.98 & 68.46 & 0.31 & 0.69 & 2.23 & 2.26 & 2.64 & 1.16 \\
\hline 24 & 1000 & 28103 & 1325.18 & 1580.36 & 2669.64 & 156.14 & 425.27 & 291.79 & 60.73 & 24.86 & 70.00 & 0.37 & 0.69 & 1.87 & 2.44 & 2.82 & 1.15 \\
\hline 25 & 1000 & 28104 & 1334.45 & 1579.47 & 2661.63 & 383.17 & 824.73 & 829.84 & 45.77 & 34.44 & 50.38 & 0.46 & 1.01 & 2.17 & 1.33 & 1.46 & 1.10 \\
\hline 26 & 1000 & 28105 & 1327.96 & 1578.58 & 2664.54 & 275.60 & 678.97 & 666.73 & 46.64 & 31.00 & 54.22 & 0.40 & 0.98 & 2.42 & 1.50 & 1.75 & 1.16 \\
\hline 27 & 1000 & 32111 & 1321.46 & 1577.69 & 2653.60 & 1775.16 & 2530.64 & 1537.68 & 62.76 & 45.62 & 53.03 & 0.70 & 0.61 & 0.87 & 1.38 & 1.16 & 0.84 \\
\hline 28 & 1000 & 32113 & 1334.45 & 1578.58 & 2664.54 & 381.19 & 2862.14 & 1401.15 & 46.98 & 21.74 & 74.01 & 0.13 & 0.49 & 3.68 & 2.16 & 3.40 & 1.58 \\
\hline 29 & 1000 & 32116 & 1327.03 & 1579.47 & 2657.98 & 370.15 & 606.02 & 363.80 & 65.70 & 38.40 & 59.91 & 0.61 & 0.60 & 0.98 & 1.71 & 1.56 & 0.91 \\
\hline 30 & 1000 & 32117 & 1334.45 & 1576.80 & 2675.46 & 121.05 & 479.18 & 245.98 & 21.65 & 23.14 & 87.00 & 0.25 & 0.51 & 2.03 & 0.94 & 3.76 & 4.02 \\
\hline 31 & 1000 & 32 & 1335.37 & 1577.69 & 2674.74 & 74.17 & 429.91 & 226.47 & 57.76 & 24.17 & 83.05 & 0.17 & 0.53 & 3.05 & 2.39 & 3.44 & 1.44 \\
\hline 32 & 1000 & 32 & 1314.03 & 1585.69 & 2659.44 & 291.52 & 576.93 & 383.83 & 83.78 & 40.58 & 52.81 & 0.50 & 0.67 & 1.32 & 2.06 & 1.30 & 0.63 \\
\hline 33 & 1000 & 32 & 1326.10 & 1579.47 & 2681.28 & 83.30 & 509.90 & 253.66 & 65.72 & 22.99 & 77.07 & 0.16 & 0.50 & 3.05 & 2.86 & 3.35 & 1.17 \\
\hline 34 & 1000 & 32 & 1331.67 & 1580.36 & 2673.28 & 53.97 & 533.77 & 245.21 & 92.19 & 24.58 & 87.09 & 0.10 & 0.46 & 4.54 & 3.75 & 3.54 & 0.94 \\
\hline 35 & 1000 & 32 & 1336.30 & 1579.47 & 2666.73 & 310.95 & 2818.89 & 1360.81 & 59.43 & 21.54 & 78.16 & 0.11 & 0.48 & 4.38 & 2.76 & 3.63 & 1.32 \\
\hline 36 & 1000 & 32 & 1326.10 & 1582.14 & 2659.44 & 336.47 & 505.61 & 328.80 & 76.61 & 50.50 & 57.29 & 0.67 & 0.65 & 0.98 & 1.52 & 1.13 & 0.75 \\
\hline 37 & 1000 & 32 & 1329.81 & 1581.25 & 2655.06 & 1618.11 & 2317.78 & 1436.99 & 68.56 & 54.25 & 51.97 & 0.70 & 0.62 & 0.89 & 1.26 & 0.96 & 0.76 \\
\hline 38 & 1000 & $32 \mathrm{~N}$ & 1332.53 & 1579.41 & 2684.86 & 84.88 & 385.57 & 186.84 & 57.23 & 21.23 & 76.81 & 0.22 & 0.48 & 2.20 & 2.70 & 3.62 & 1.34 \\
\hline 39 & 1000 & $32 \mathrm{~N}$ & 1328.82 & 1581.19 & 2682.68 & 477.89 & 2401.55 & 976.94 & 38.72 & 19.72 & 85.45 & 0.20 & 0.41 & 2.04 & 1.96 & 4.33 & 2.21 \\
\hline 40 & 1000 & $32 \mathrm{~N}$ & 1322.33 & 1579.41 & 2668.13 & 108.06 & 398.76 & 182.36 & 43.46 & 19.11 & 83.93 & 0.27 & 0.46 & 1.69 & 2.27 & 4.39 & 1.93 \\
\hline 41 & 1000 & $32 \mathrm{~N}$ & 1327.89 & 1581.19 & 2667.41 & 376.96 & 1752.54 & 723.56 & 35.36 & 20.83 & 72.03 & 0.21 & 0.41 & 1.92 & 1.70 & 3.46 & 2.04 \\
\hline 42 & 1000 & $32 \mathrm{~N}$ & 1329.75 & 1578.52 & 2659.39 & 524.79 & 1433.55 & 735.56 & 64.95 & 25.78 & 67.08 & 0.36 & 0.51 & 1.40 & 2.52 & 2.60 & 1.03 \\
\hline 43 & 1000 & $32 \mathrm{~N}$ & 1332.53 & 1581.19 & 2663.03 & 561.28 & 2024.36 & 1265.55 & 43.08 & 23.78 & 59.19 & 0.28 & 0.63 & 2.25 & 1.81 & 2.49 & 1.37 \\
\hline
\end{tabular}

Figure 3.15 | Raman spectral parameters of synthesized 3D graphene samples at different

\section{temperatures.}




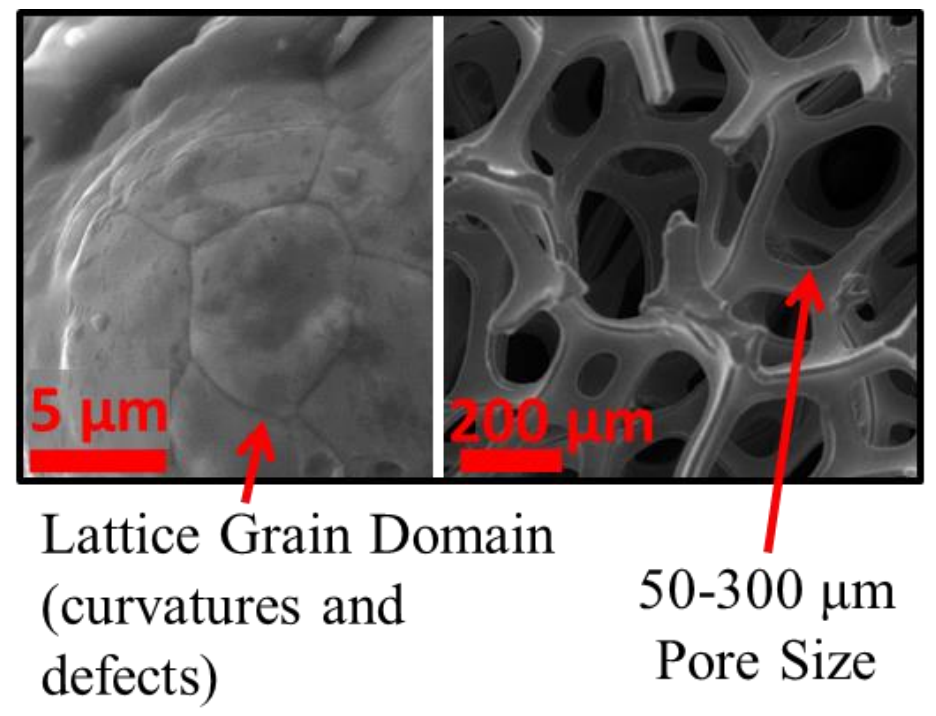

Figure 3.16 | SEM images Show Morphology of a Ni Foam.

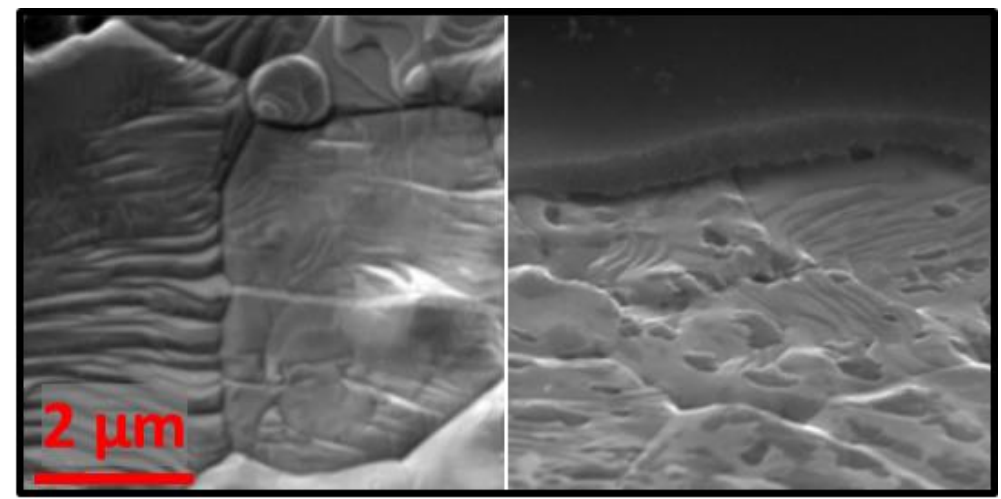

Figure 3.17 | After the Growth, Graphene Coat Has a Morphology Similar to the Morphology of the Substrate (Ni Foam). 


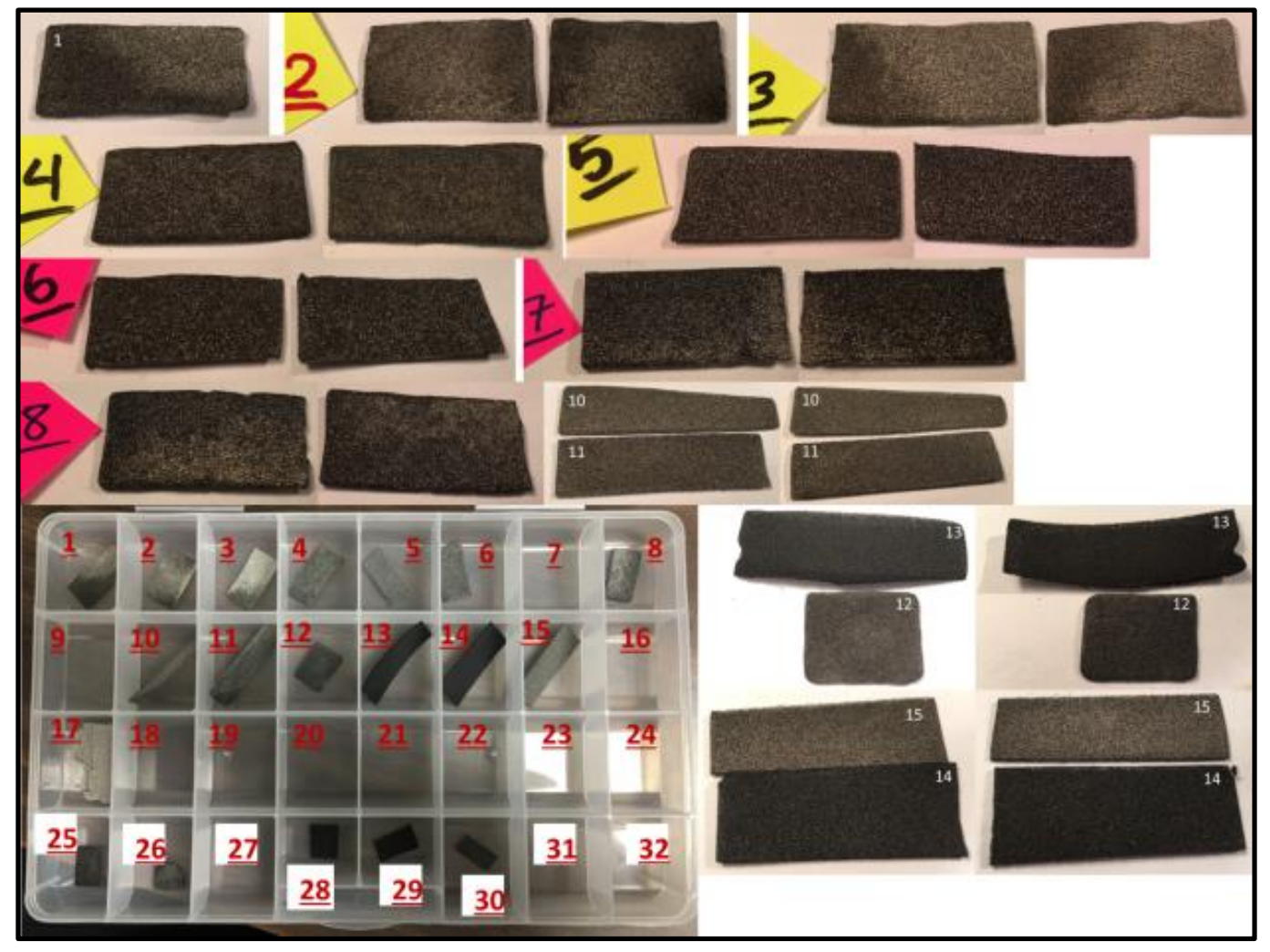

Figure 3.18 | Photograph of as-prepared 3D graphene foams synthesized under different growth conditions. 


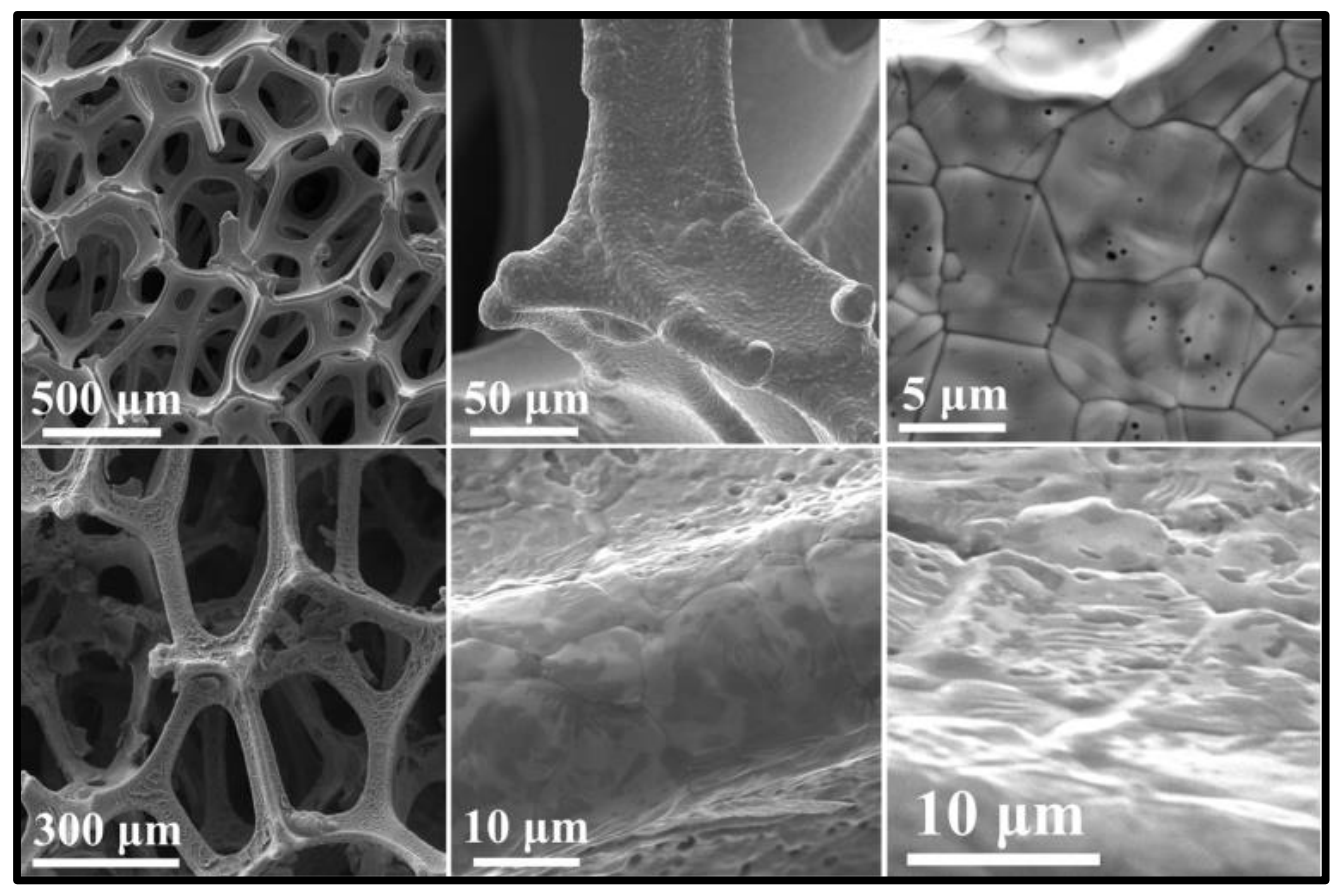

Figure 3.19 | Scanning Electron Microscopy (SEM) imaging of Ni foam before (top row) and after (bottom row) the graphene growth.

SEM images reveal (Figure 3.19 and 3.16) that the Nickel foam had a porous interconnected 3D network with a pore size of $\sim 200$ to $\sim 300 \mu \mathrm{m}$ in diameter. The TEM images further revealed that both nitrogen-doped and pure 3D graphene samples are consist of multilayer graphene. Figure 3.20 is a low-resolution TEM image of a graphene sheet with folded areas and indicated the growth of multi-layered graphene without the amorphous carbon. Furthermore, High-Resolution Transmission Electron Microscopy (HRTEM) revealed that graphene sheets containing 5-30 layers. 


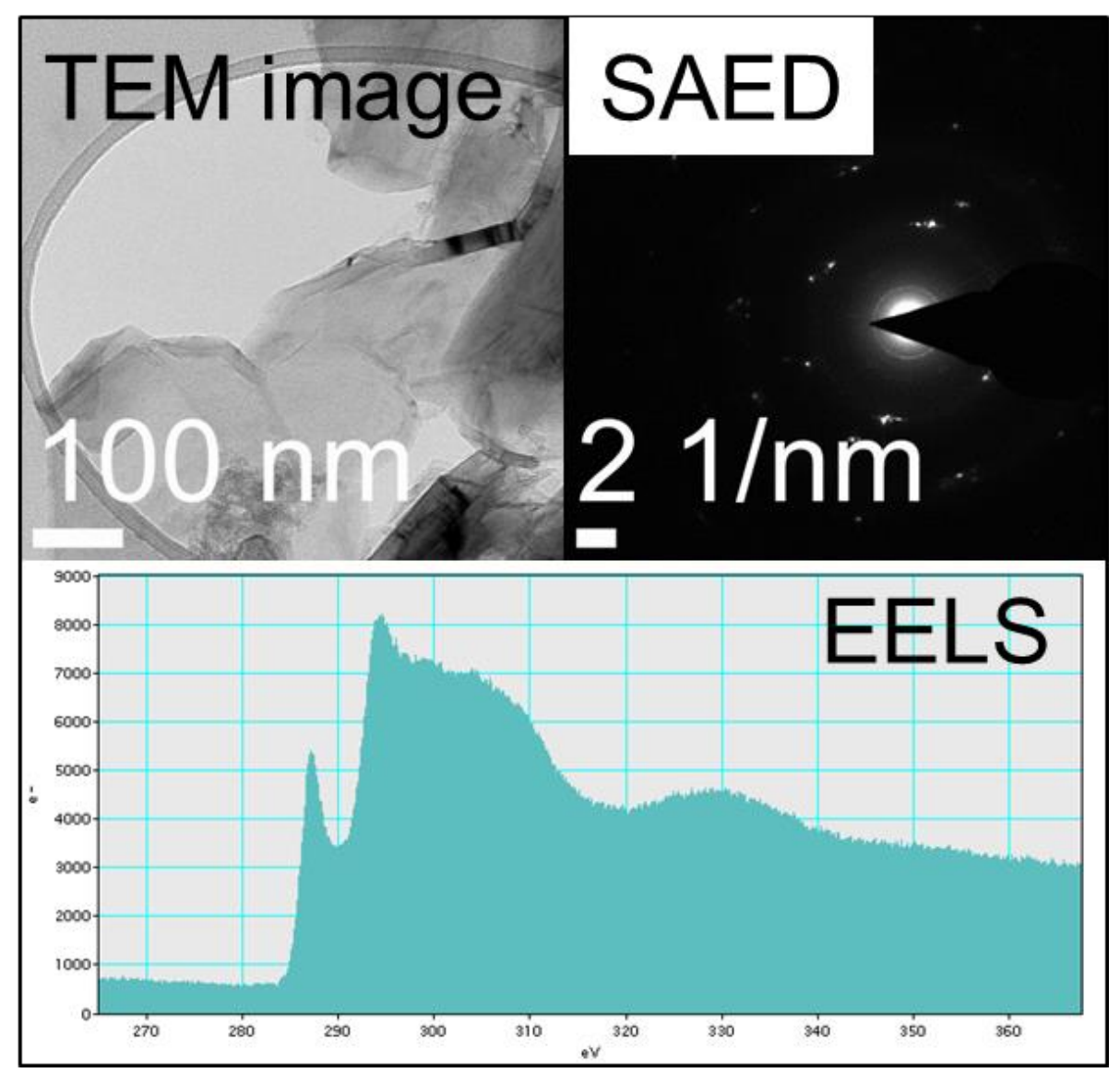

Figure 3.20 | a, Transmission Electron Microscopy (TEM) image, b, Selected Area Electron Diffraction (SAED) pattern, and c, Electron Energy Loss Spectroscopy (EELS) of pure 3D graphene foam prepared by pyrolysis of Citric Acid as the carbon source. 


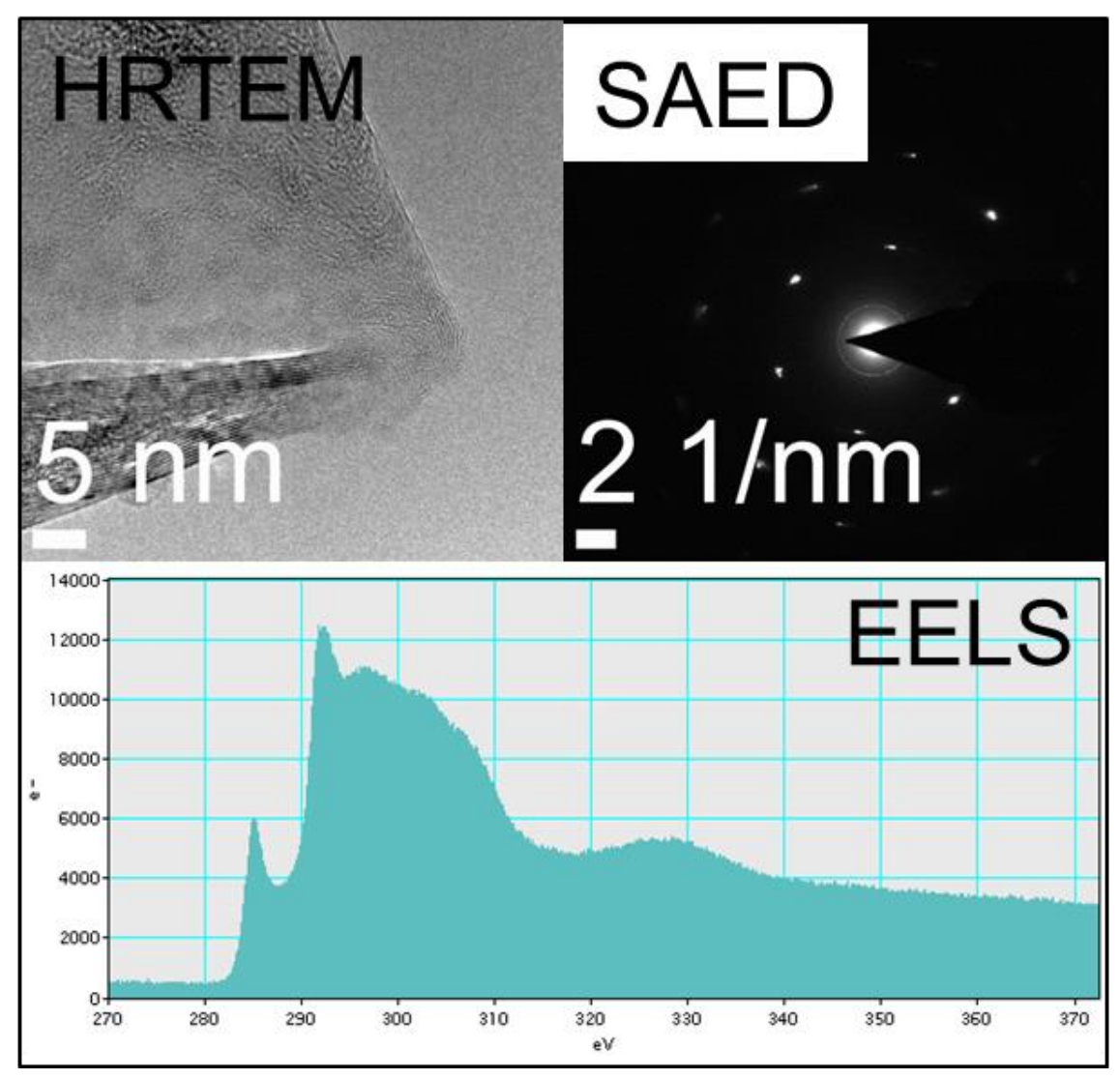

Figure $3.21 \mid$ a, Transmission Electron Microscopy (TEM) image, b, Selected Area Electron Diffraction (SAED) pattern, and c, Electron Energy Loss Spectroscopy (EELS) of Nitrogen-doped 3D graphene foam prepared by caffeine as the carbon source. 

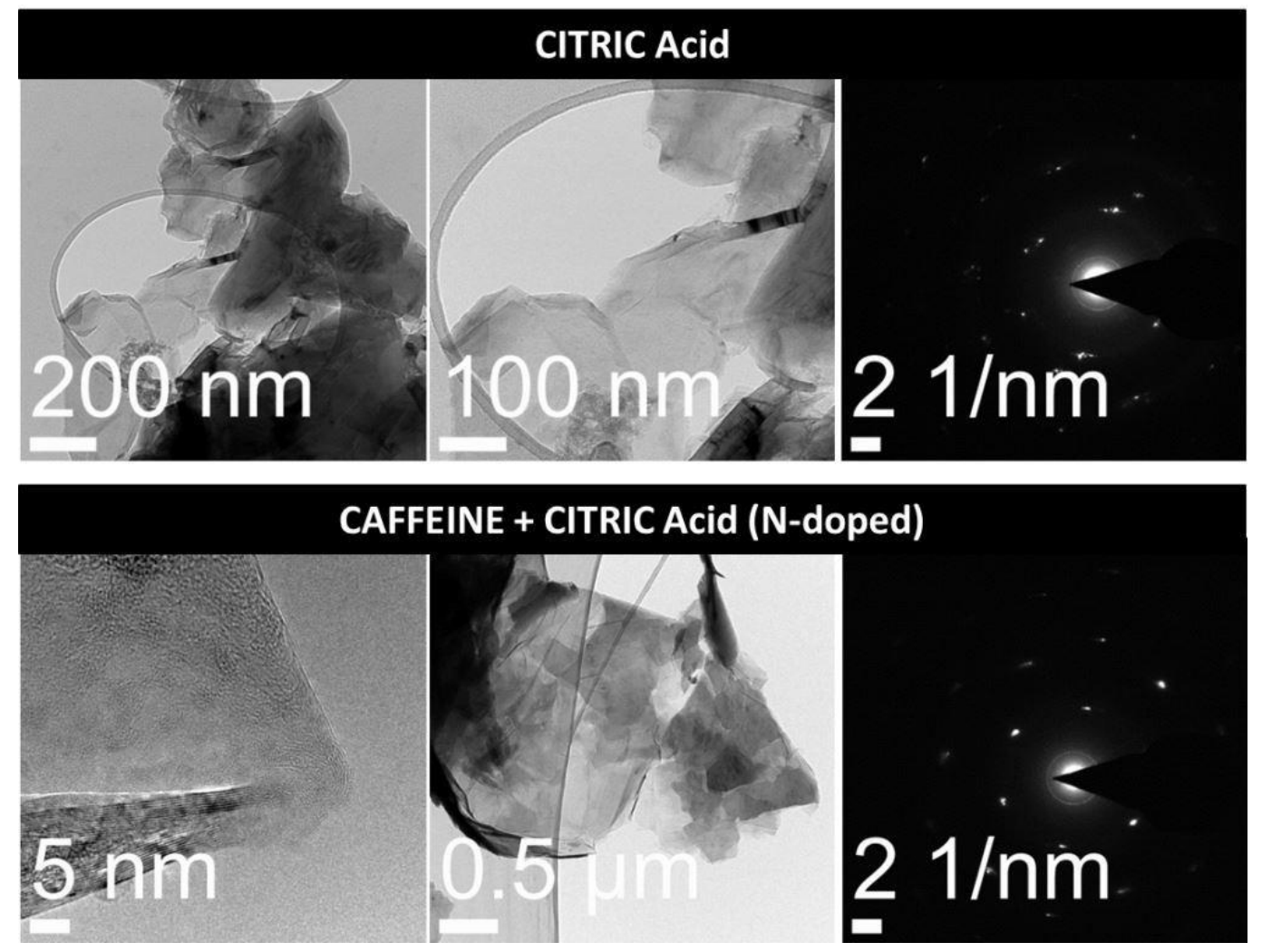

Figure 3.22 | TEM Analysis of Top: Pure 3D Graphene (precursor: Citric Acid) and Bottom: In Situ Nitrogen doped 3D Graphene (precursor: Citric Acid + Caffeine) Furthermore, SAED pattern of the 3D graphene foam indicated the presence of nickel nanoparticles on top of the graphene layers, which resulted in the formation of nanocages on the surface of the graphene structure. 


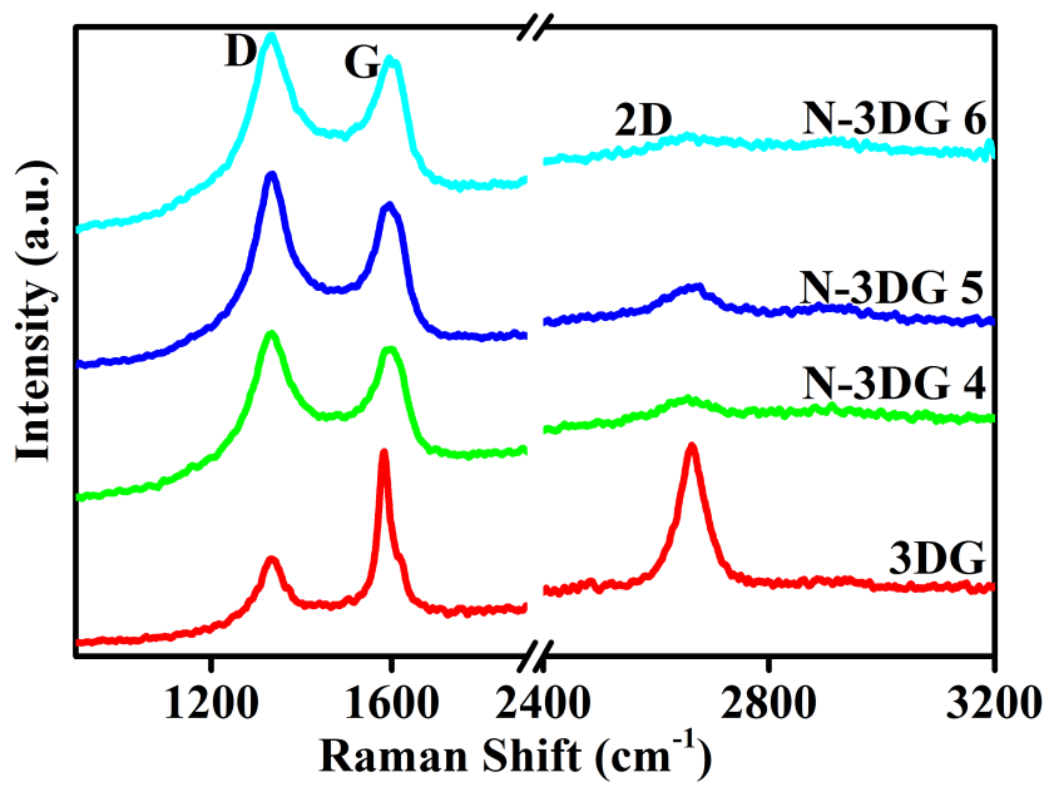

Figure 3.23 | Raman spectroscopy of nitrogen-doped 3D graphene foam (three upper spectra) and Raman spectrum of pure 3D graphene foam (red) all grown at $1000^{\circ} \mathrm{C}$.

Chemical compositions of the nitrogen-doped 3D graphene foams were investigated by X-ray Photoelectron Spectroscopy (XPS). Figures 3.25 and 3.26 are high-resolution XPS spectra for the sample synthesized at $1000{ }^{\circ} \mathrm{C}$ with urea and citric acid as the sources of carbon and nitrogen.

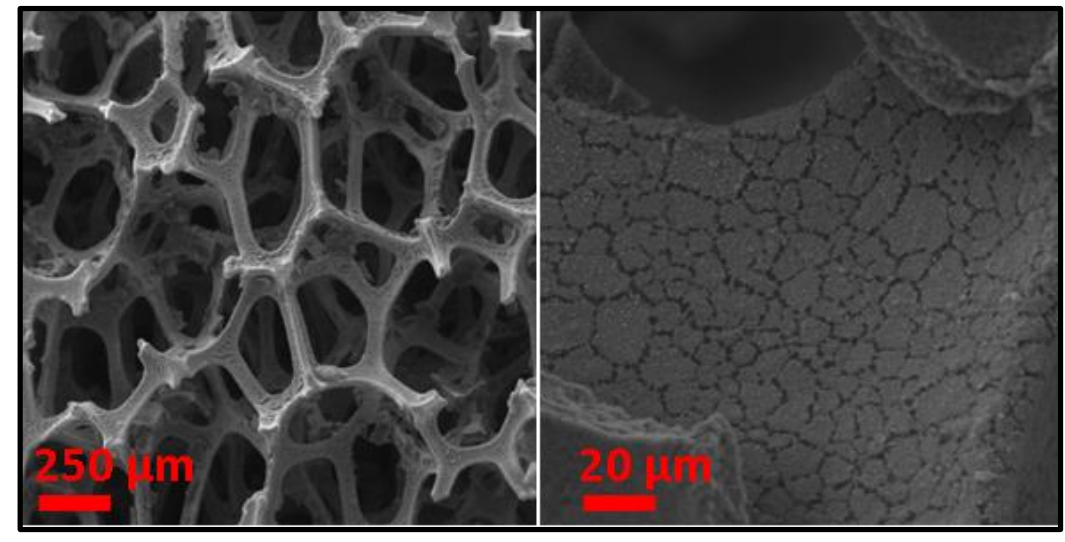

Figure 3.24 | SEM Images of a Nitrogen Doped Sample 


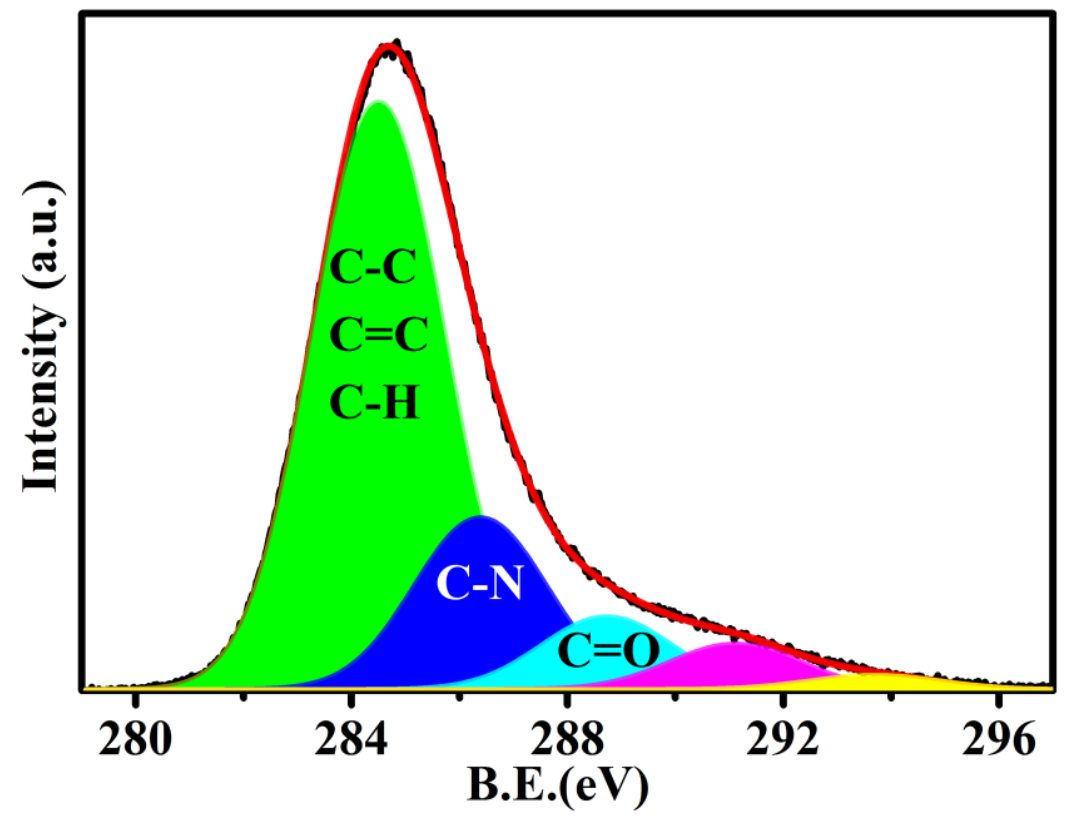

Figure 3.25 | Deconvoluted high-resolution C (1s) X-ray photoelectron spectroscopy (XPS) spectra of Nitrogen-doped 3D graphene foam using Urea as the carbon source.

A detailed analysis of XPS spectra $[148,149]$ revealed the existence of some oxygencontaining functional groups in addition to the nitrogen on the 3D graphene foam surface. Moreover, deconvolutions of the spectra indicate components related to bound hydroxide groups $\left(\mathrm{OH}^{-}\right)$. 


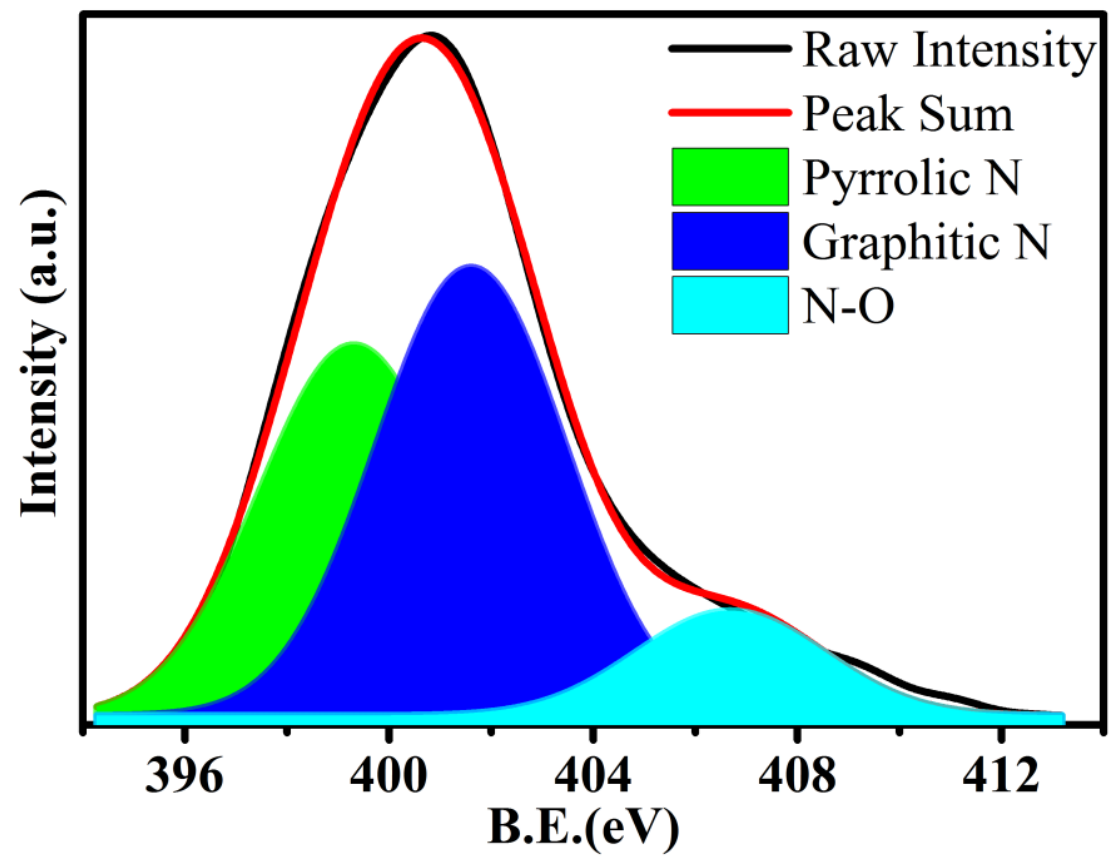

Figure 3.26 | Deconvoluted high-resolution N (1s) X-ray photoelectron spectroscopy (XPS) spectra of Nitrogen-doped 3D graphene foam prepared by Urea as the carbon source.

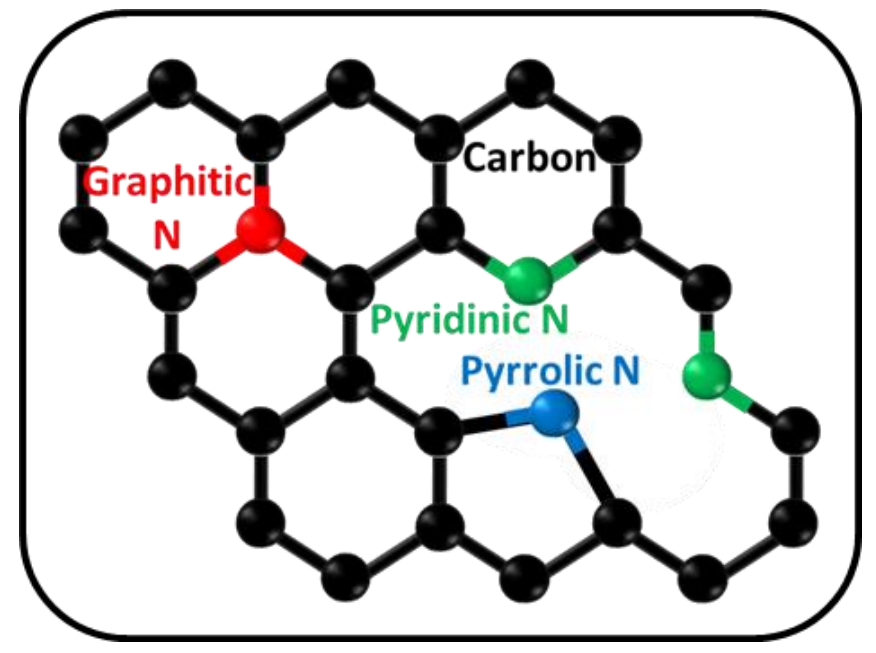

Figure 3.27 | nitrogen-bonding configurations in the nitrogen-doped carbon

The peak analysis of deconvoluted XPS curves indicated that about 3\% nitrogen is incorporated into the graphene surface. Furthermore, electrochemical catalytic activity 
testing ${ }^{3}$ to reduce $\mathrm{CO}_{2}$ showed a significant increase in catalytic activity of Ni-doped carbon when $3 \mathrm{D}$ graphene network is used as the core-shell $\left(\mathrm{CO}_{2}\right.$ reduction to $\left.\mathrm{CO}\right)$.

Figure 3.28 shows a comparison of the catalytic activity of Nickel foam; threedimensional graphene network on nickel foam, Carbon doped with Nitrogen (polyaniline) with core-shell of 3D graphene, and silver film. In the electrochemical testing $\mathrm{Ag} / \mathrm{AgCl}$ (3.0 $\mathrm{M} \mathrm{KCl)} \mathrm{was} \mathrm{used} \mathrm{as} \mathrm{the} \mathrm{reference} \mathrm{electrode,} \mathrm{Pt}$ mesh as counter electrode and the sample as the working electrode. Moreover, the electrolyte was $\mathrm{CO}_{2}$-saturated $0.1 \mathrm{M}$ $\mathrm{KHCO}_{3}$ and the $\mathrm{CO}_{2}$ reduction products were measured by gas chromatography.

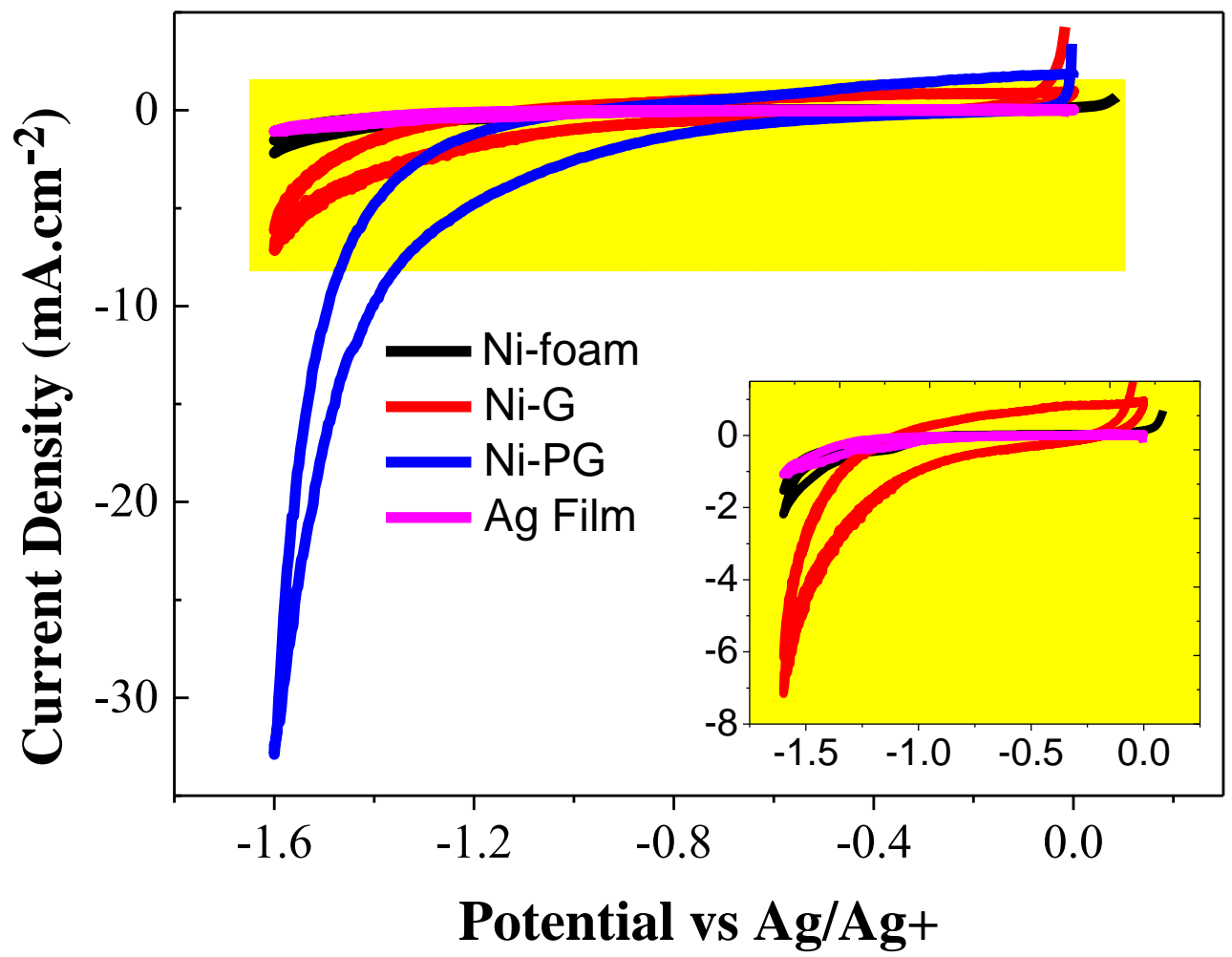

Figure 3.28 | Catalytic activity of Nickel foam, Three-dimensional graphene network on nickel foam (Ni-G), Carbon doped with Nitrogen (polyaniline) with core-shell of 3D graphene (Ni-PG), and Silver film (Ag Film).

\footnotetext{
${ }^{3}$ Performed by Dr. Bijandra Kumar (Conn Center for Renewable Energy Research)
} 


\subsection{Phosphorene}

The high-pressure evolution of Raman frequency of phosphorene (few-layer black phosphorus) from ambient pressure to $\sim 14 \mathrm{GPa}$ was collected at room temperature. High pressure was generated by a gas membrane driven "diamond anvil cell” (Diacell ${ }^{\circledR}$ HeliosDAC Almax easyLab) equipped with $800 \mu \mathrm{m}$ culet diamonds. Inconel gasket with $10 \mathrm{~mm}$ in diameter and $0.20 \mathrm{~mm}$ thickness were pre-indented down to $0.05 \mathrm{~mm}$. Micro Electrical discharge machining (EDM) (Hylozoic Products) was used for drilling a small hole (0.3 $\mathrm{mm}$ in diameter) for sample loading.

In-situ high-pressure Raman measurements were conducted at room temperature using the diamond anvil. Each sample was excited with $\mathrm{He}-\mathrm{Ne}(633 \mathrm{~nm})$ red laser in a $180^{\circ}$ backscattering geometry using a Leica microscope with a 50X Nikon objective lens ( $\mathrm{T}$ Plan EPI SLWD, Working Distance $=22 \mathrm{~mm}$ ). Renishaw inVia spectrometer with a 1800 lines per millimeter grating, and equipped with a CCD detector, was used for Raman spectroscopy. The Raman spectra were collected at various pressures during compression and decompression.

Ruby fluorescence R2-line shifts were used for pressure calibration. This method is known to have an error of $\pm 5 \%$. The ruby fluorescence was measured with same Renishaw spectrometer and He-Cd (442 nm) blue laser (IK series, Kimmon Koha Co.) during compression and decompression. 


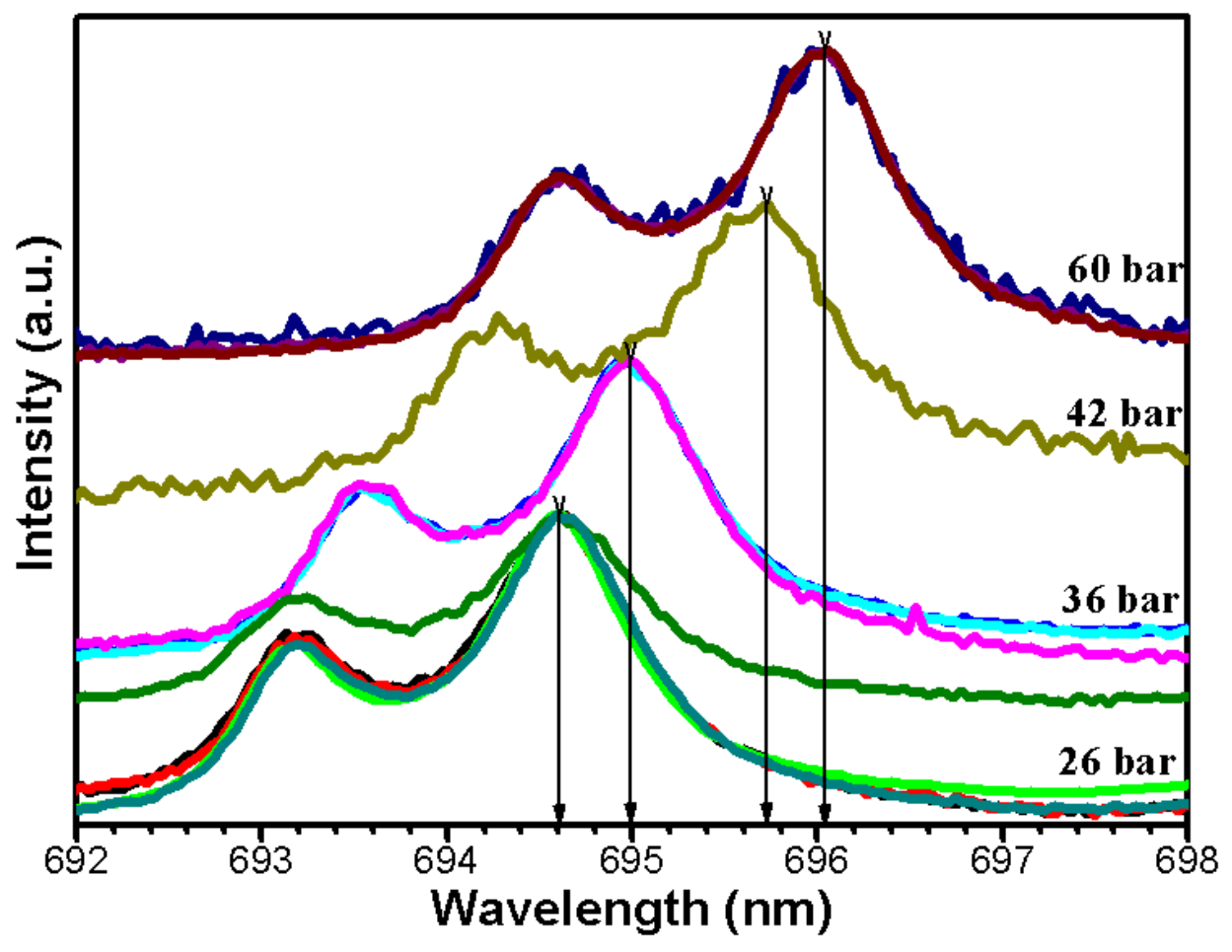

Figure 3.29 | Ruby fluorescence spectra at different gas membrane pressures (measured by gas controller unit). These pressures correspond to the actual pressures inside the DAC. 


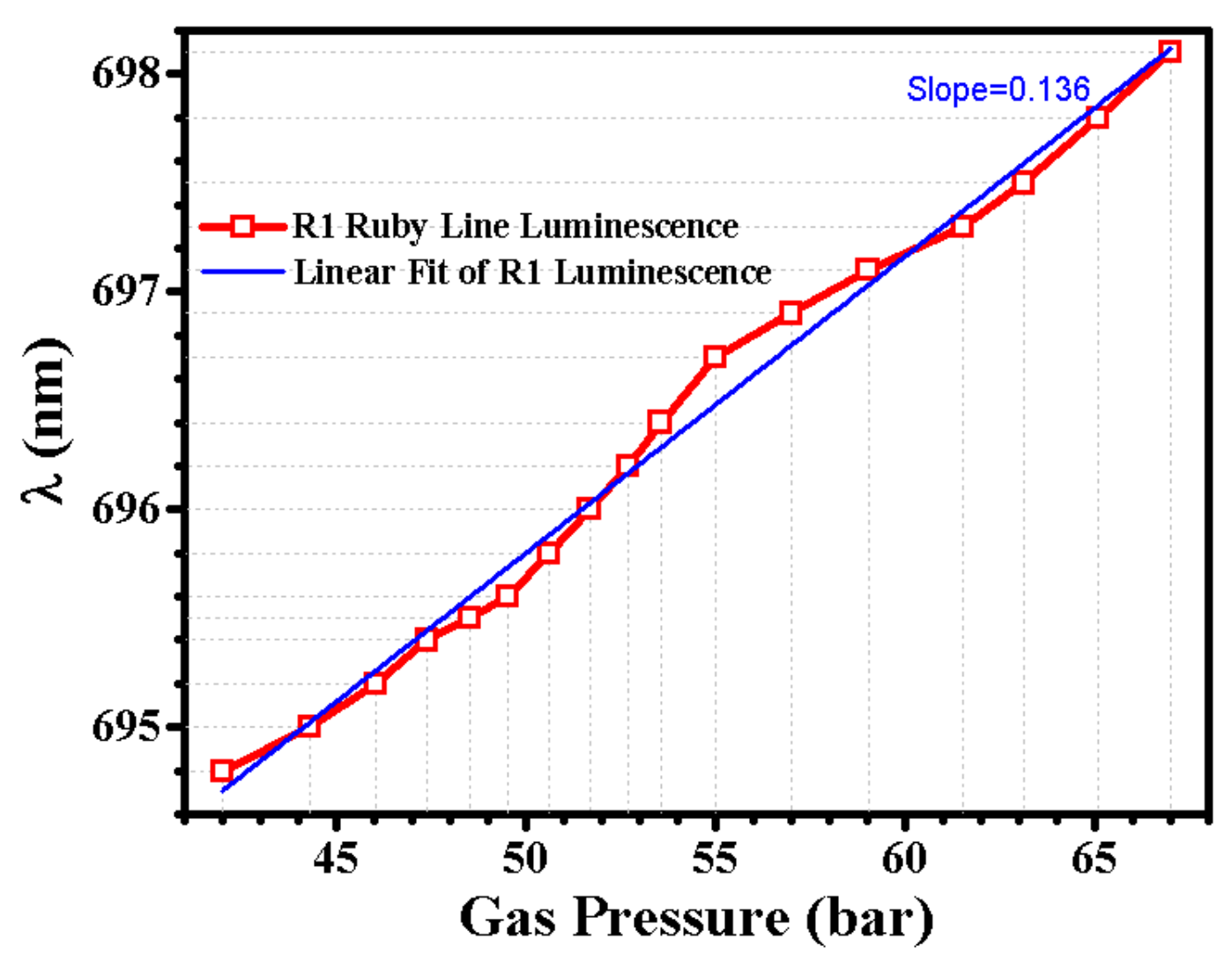

Figure 3.30 | The ruby fluorescence R2 line peak position as a function of pressure inside the gas membrane (measured by gas controller unit).

The pressure of gas membrane (which drive the piston of the cell) was calibrated against the pressure inside the DAC. For each high-pressure experiment, small pieces of phosphorene samples were loaded into the gasket hole under an optical microscope (AmScope Trinocular Stereo Zoom) with long working distance objective lenses. It was visually ensured that the phosphorene samples were floating in the middle of the hole and were not in contact with the gasket or the diamond. We tested two solutions Methanol:Ethanol:DI-water (16:4:1) and Dimethylformamide (DMF) separately as the hydrostatic pressure media. The transparency of diamonds to the broad range of electromagnetic waves allows us to perform a series of $i n-s i t u$ high-pressure 
measurement including precise pressure calibration from ruby photoluminescence R2 line, and Raman spectroscopy that resulted in the observation of phase transition in multilayered BP at high pressures.

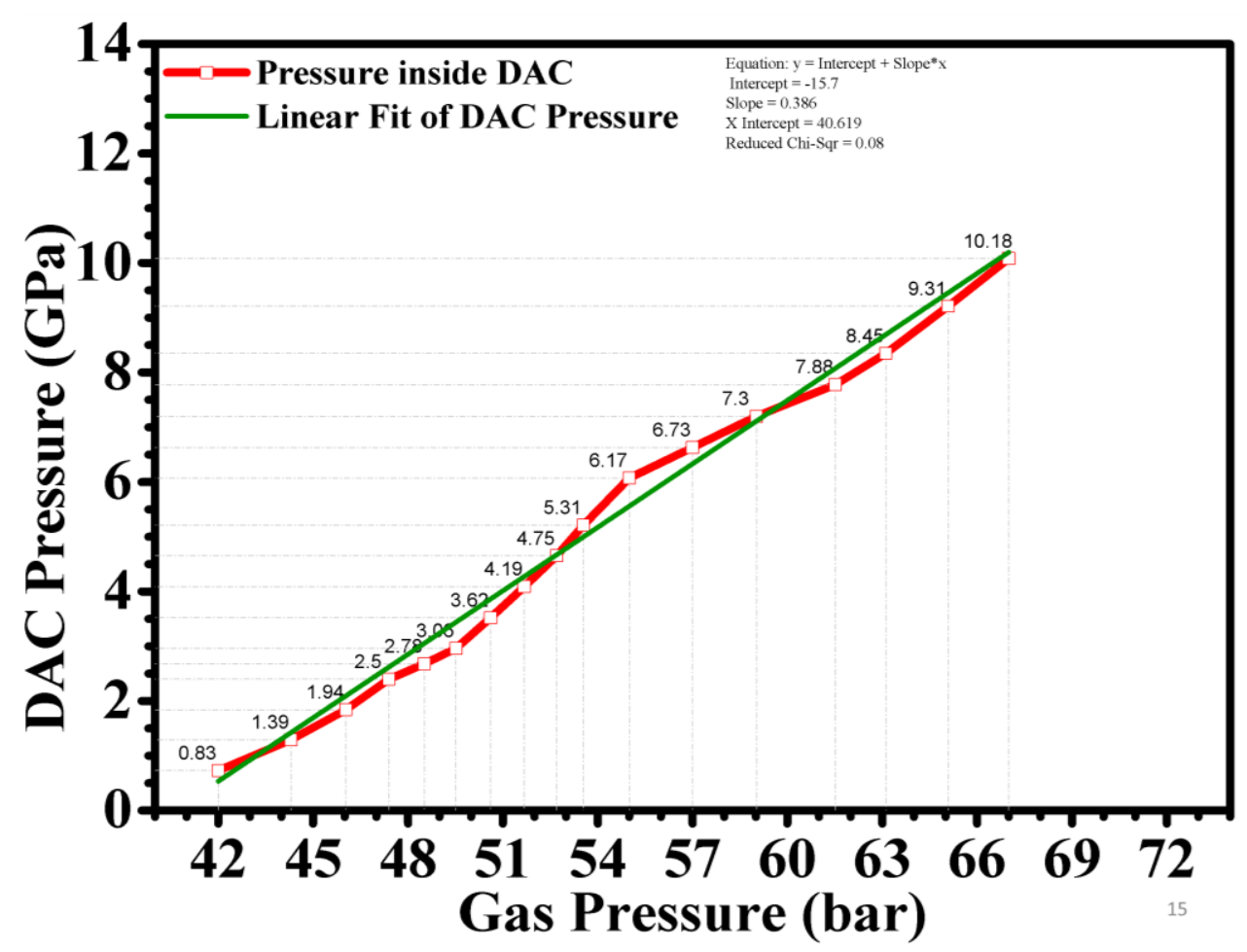

Figure 3.31 | Pressure inside the diamond anvil cell as a function of gas membrane pressure.

The Raman spectra at ambient pressure of few layer black phosphorus samples are shown in Figure 3.32. The prominent Raman spectrum peaks at $360 \mathrm{~cm}^{-1}, 440 \mathrm{~cm}^{-1}$, and $468 \mathrm{~cm}^{-}$ ${ }^{1}$ which were assigned to the $A_{g}^{1}$ (out-of-plane), $B_{2 g}$ (in-plane along the zigzag direction), and $A_{g}^{2}$ (in-plane along the armchair direction) vibrational modes respectively [150-152]. It has been known that both the bulk BP and phosphorene show these three Raman peaks which suggesting that black phosphorus stays crystalline after the exfoliation process $[150,153-156]$. 


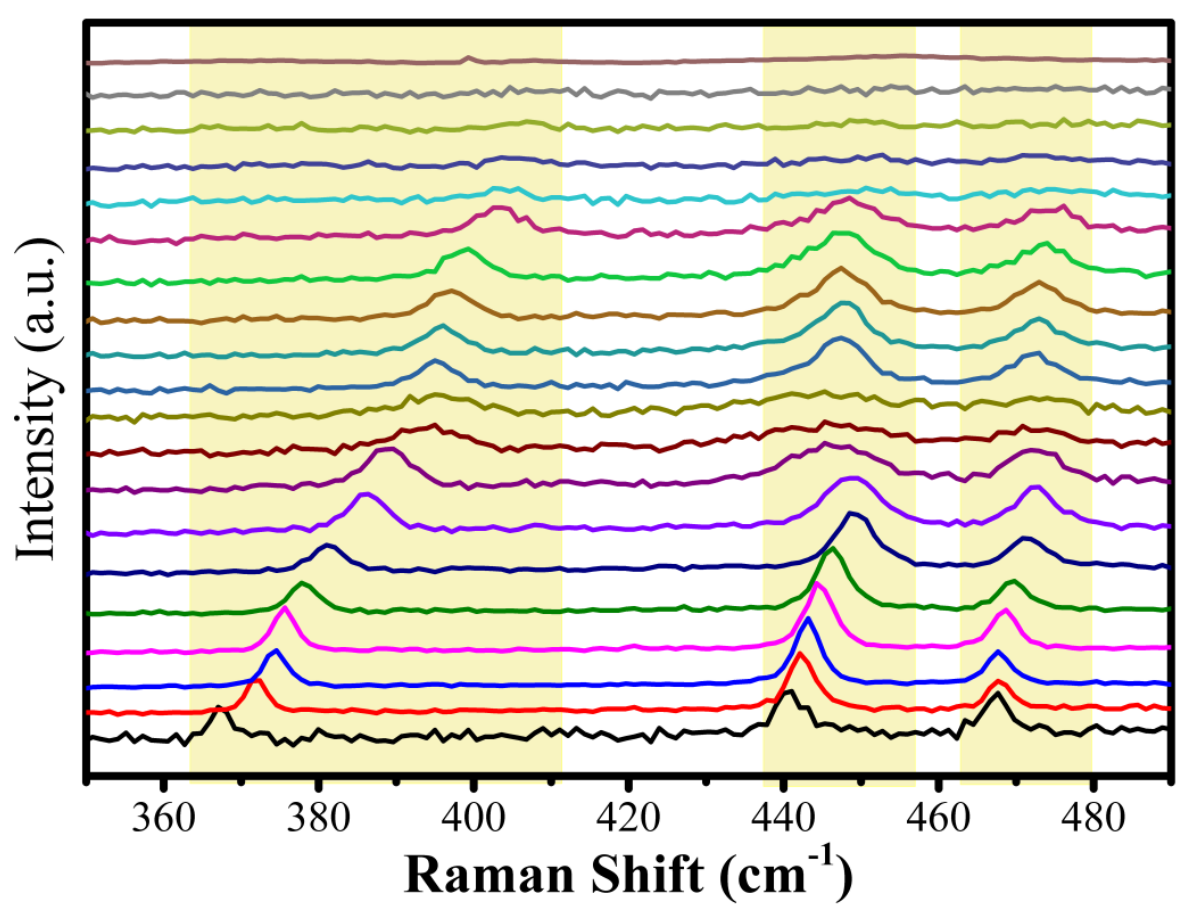

Figure 3.32 | Raman spectra of a few-layer black phosphorus (sample no. 1) under pressure range of $0 \mathrm{GPa}$ (bottom spectrum) to $13.4 \mathrm{GPa}$ (top spectrum) with vertical displacements for clarity.

As shown in Figure 3.32 and Figure 3.33, with the increase in pressure, all phonon modes shift towards higher frequencies (blue shift) with gradually decreasing intensity. The frequencies of Raman modes of a few-layer BP sample as a function of applied hydrostatic pressure are shown in Figure 3.34. The application of pressure was found to modify the vibrational properties of few-layer black phosphorus strongly. 


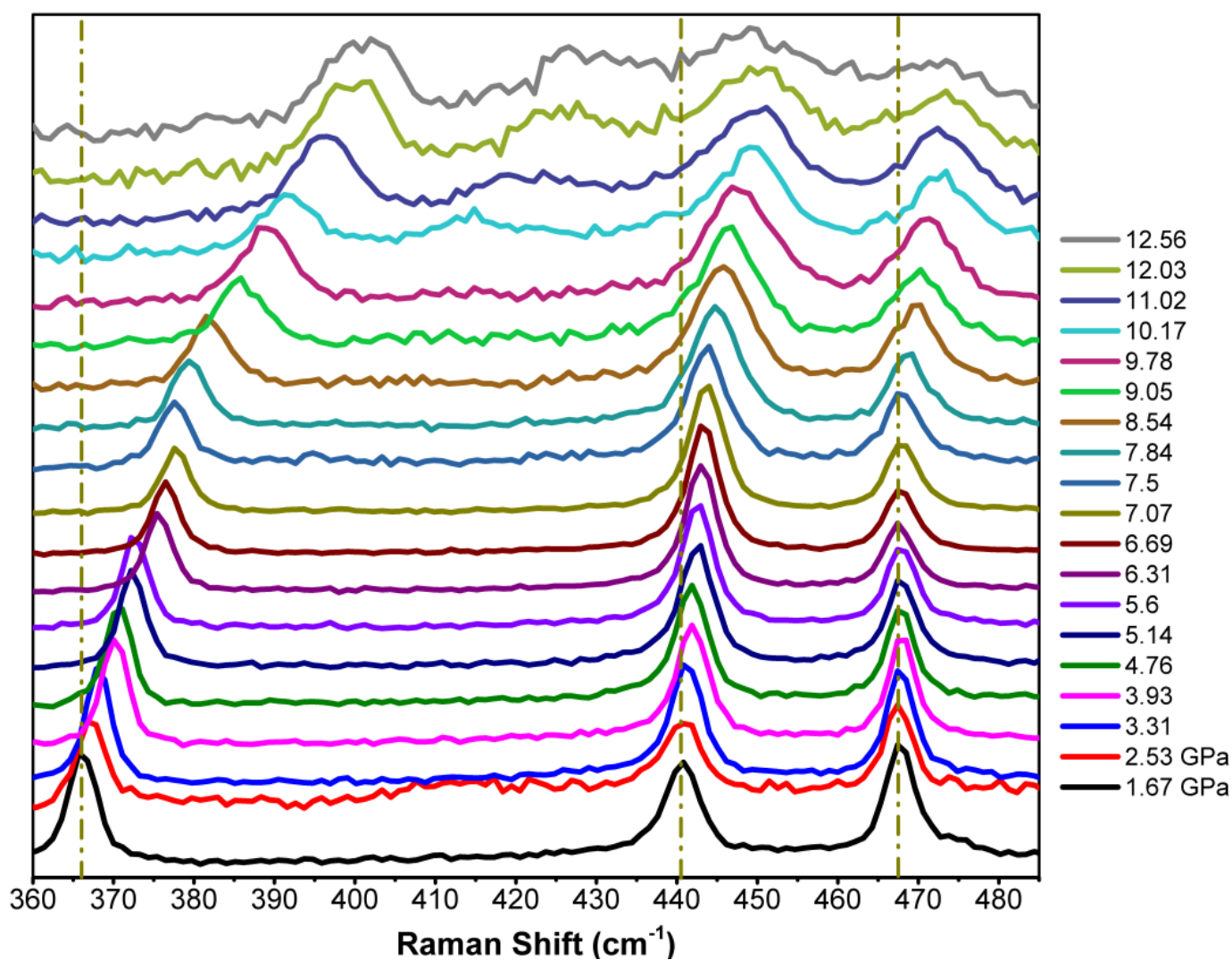

Figure 3.33 | Raman spectra of a few-layer black phosphorus under pressure range of 0 $\mathrm{GPa}$ (bottom spectrum) to $12.56 \mathrm{GPa}$ (top spectrum) with vertical displacements for clarity.

The crystal structure of bulk black phosphorus at normal conditions is orthorhombic (A11) and belongs to the space group Cmce (No. 64) and point group $D_{2 h}^{18}(\mathrm{mmm})$ $[153,157]$. It has been known that the bulk black phosphorus orthorhombic (A11) crystal structure exhibits a sequence of transitions under high pressure. First, at $\sim 5.2$ GPa black phosphorus transforms from the orthorhombic to the rhombohedral (A7) crystal phase with $10 \%$ reduction in volume and then with $3.7 \%$ volume reduction at $\sim 10 \mathrm{GPa}$ it transforms to the simple-cubic structure [153]. The rhombohedral BP is a semimetal and, simple-cubic BP is a metallic material and shows superconductivity at low temperature 
[61-63]. Bulk BP with orthorhombic crystal phase has eight atoms in a unit cell, which half of them (four) are in the primitive unit cell. Therefore, it has 12 vibrational (phonon) modes at the $\Gamma$ point in the Brillouin zone [157]:

$$
\Gamma_{\mathrm{Bulk}}=2 A_{g}+B_{1 g}+B_{2 g}+2 B_{3 g}+A_{1 u}+2 B_{1 u}+2 B_{2 u}+B_{3 u}
$$

which consist of five Raman-active $\left(A_{g}^{1}, A_{g}^{2}, B_{1 g}, B_{2 g}\right.$, and $\left.B_{3 g}\right)$, three infrared-active $\left(B_{1 u}, B_{2 u}\right.$, and $\left.B_{3 u}\right)$, one silent $\left(A_{u}\right)$, and three acoustic $\left(A_{1 u}, B_{1 u}\right.$, and $\left.B_{2 u}\right)$ phonon modes.

The rhombohedral (A7) lattice with two atoms per primitive unit cell has three Raman active modes at zone-center, that is, one $A_{1 g}$, mode and a two-fold degenerate $E_{g}$ mode.

According to the classical Placzek approximation [157-160], the Raman intensity of a phonon mode is proportional to $\left|e_{i} \cdot \tilde{R} \cdot e_{s}^{T}\right|^{2}$ where $e_{i}$ and $e_{s}$ are the electric polarization vectors of the incident laser and scattered light, respectively, and $\tilde{R}$ is the Raman tensor of the phonon mode. Phonon modes can be observed by Raman spectroscopy only when $\left|e_{i} \cdot \tilde{R} \cdot e_{s}^{T}\right|^{2}$ is not zero. By denoting the zigzag and armchair directions as the $\mathrm{x}-$ and $\mathrm{z}-$ axis, and the out-of-plane direction as the y-axis, the calculated (via the group theory analysis) Raman tensors $\tilde{R}$ of $A_{g}, B_{1 g}, B_{2 g}$, and $B_{3 g}$ modes are [158]:

$$
\begin{array}{ll}
\tilde{R}\left(A_{g}\right)=\left(\begin{array}{lll}
a & 0 & 0 \\
0 & b & 0 \\
0 & 0 & c
\end{array}\right) & \tilde{R}\left(B_{1 g}\right)=\left(\begin{array}{ccc}
0 & d & 0 \\
d & 0 & 0 \\
0 & 0 & 0
\end{array}\right) \\
\tilde{R}\left(B_{2 g}\right)=\left(\begin{array}{lll}
0 & 0 & e \\
0 & 0 & 0 \\
e & 0 & 0
\end{array}\right) & \tilde{R}\left(B_{3 g}\right)=\left(\begin{array}{ccc}
0 & 0 & 0 \\
0 & 0 & f \\
0 & f & 0
\end{array}\right)
\end{array}
$$


Where $a-f$ are non-zero terms while other terms are either zero or negligible due to symmetry. In the typical experimental back-scattering laser geometry (y in and y out for the laser), the electric polarization vectors of the incident and scattered light $e_{i}$ and $e_{s}$ are in-plane (the x-z plane) in other words, direction of incident laser beam is perpendicular to the layers plane of BP sample. By setting the polarization angle of the incident (scattered) light as $\theta(\gamma)$ with respect to the x-axis, we have $e_{i}=(\cos \theta, 0, \sin \theta)$ and $e_{s}=(\cos \gamma, 0, \sin \gamma)$. For Raman intensity, we have:

$$
\mathrm{I} \propto\left|e_{i} \cdot \tilde{R} \cdot e_{s}^{T}\right|^{2}
$$

By substituting $e_{i}$ and $e_{s}$ we have:

$$
\mathrm{I} \propto\left|(\cos \theta, 0, \sin \theta) \tilde{R}\left(\begin{array}{c}
\cos \gamma \\
0 \\
\sin \gamma
\end{array}\right)\right|^{2}
$$

By applying the obtained forms of Raman tensor of each mode, we can get the Raman intensities for each mode:

$$
\begin{gathered}
\boldsymbol{I}_{\boldsymbol{A}_{g}} \propto a^{2}\left|\cos \theta \cos \gamma+\frac{\mathrm{c}}{\mathrm{a}} \sin \theta \sin \gamma\right|^{2} \\
\boldsymbol{I}_{\boldsymbol{B}_{2 g}} \propto e^{2} \sin ^{2}(\theta+\gamma) \\
\boldsymbol{I}_{\boldsymbol{B}_{1 \boldsymbol{g}}}=\boldsymbol{I}_{\boldsymbol{B}_{3 g}}=0
\end{gathered}
$$

Therefore, $B_{1 g}$ and $B_{3 g}$ cannot be observed, and only $A_{g}$ and $B_{2 g}$ modes can be observed. Furthermore, the symmetries of N-layer (NL) BP (where N is the number of layers) are slightly different from those of bulk BP. Odd NL BP belongs to space group Pmna (No. 53) and point group $D_{2 h}^{7}(\mathrm{mmm})$; even NL BP belongs to space group Pmca 
(No. 57) and point group $D_{2 h}^{11}(\mathrm{mmm})$. Although NL systems belong to different space groups from the bulk BP, all of them share the same point group $D_{2 h}(\mathrm{mmm})$.

Consequently, the symmetry classification of Raman modes and the forms of their Raman tensors remain unchanged for any thickness [157].

Bulk BP at normal pressure:

Crystal structure Orthorhombic (A11)

Space group

Cmce (No. 64)

Point group

$D_{2 h}^{18}$

Number of atoms in primitive unit cell

4 atoms

$\Gamma_{\mathrm{Bulk}}=2 A_{g}+B_{1 g}+B_{2 g}+2 B_{3 g}+A_{1 u}+2 B_{1 u}+2 B_{2 u}+B_{3 u}$

Raman active modes

$2 A_{g}+B_{1 g}+B_{2 g}+2 B_{3 g}$

Infrared (IR) active modes

$B_{1 u}+B_{2 u}$

Acoustic modes

$B_{1 u}+B_{2 u}+B_{3 u}$

Silent mode

$A_{1 u}$

Few-layer BP at normal pressure:

a) With (AA or AB stacking, and odd \# of layers) or (AA stacking, and even \# of layers):
Space group
Pmna (No. 53)
Point group
$D_{2 h}^{7}(m m m)$ 


$$
\begin{array}{ll}
\begin{array}{l}
\Gamma=2 \mathrm{~N}\left(A_{g}+B_{2 g}+B_{1 u}+B_{3 u}\right)+\mathrm{N}\left(B_{1 g}+B_{3 g}+A_{1 u}+B_{2 u}\right) \\
\text { Raman active modes } \\
\text { Infrared (IR) active modes }
\end{array} & (2 \mathrm{~N}-1)\left(B_{1 u}+B_{2 g}\right)+\mathrm{N}\left(B_{1 g}+B_{3 g}\right)+(2 \mathrm{~N}-1) B_{2 u} \\
\text { Acoustic modes } & B_{1 u}+B_{2 u}+B_{3 u} \\
\text { Silent modes } & \mathrm{N} A_{1 u}
\end{array}
$$

b) With $\mathrm{AB}$ stacking, and even \# of layers:

$$
\begin{aligned}
& \begin{array}{ll}
\text { Space group } \quad \text { Pmca (No. 57) }
\end{array} \\
& \text { Point group } \quad D_{2 h}^{11} \\
& \Gamma=2 \mathrm{~N}\left(A_{g}+B_{2 g}+B_{1 u}+B_{3 u}\right)+\mathrm{N}\left(B_{1 g}+B_{3 g}+A_{1 u}+B_{2 u}\right) \\
& \text { Raman active modes } \quad 2 \mathrm{~N}\left(A_{g}+B_{2 g}\right)+\mathrm{N}\left(B_{1 g}+B_{3 g}\right) \\
& \text { Infrared (IR) active modes } \quad(2 \mathrm{~N}-1)\left(B_{1 u}+B_{3 u}\right)+(2 \mathrm{~N}-1) B_{2 u} \\
& \text { Acoustic modes } \quad B_{1 u}+B_{2 u}+B_{3 u} \\
& \text { Silent modes } \quad \mathrm{N} A_{1 u}
\end{aligned}
$$

Bulk BP at a pressure range of $\sim 4.5$ to $\sim 10 \mathrm{GPa}$ :

Crystal structure

Space group
Rhombohedral (A7)

R⿳亠丷厂m (No. 166) 
Point group $\quad D_{3 d}^{5}$

Number of atoms in primitive unit cell 2 atoms

$\Gamma_{\text {Bulk }}($ Rhombohedral $)=3\left(A_{g}+E_{g}+B_{1 u}+B_{2 u}\right)$

Raman active modes

Infrared (IR) active modes

Acoustic modes

Silent mode
$3\left(A_{g}+E_{g}\right)$

$2\left(B_{1 u}+B_{2 u}\right)$

$B_{1 u}+B_{2 u}$

None

Bulk BP at a pressure above $10 \mathrm{GPa}$ :

Crystal structure

Space group

Point group

Number of atoms in primitive unit cell
Simple cubic

$\operatorname{Pm} \overline{3} \mathrm{~m}$

$O_{h}^{1}$

1 atom

$$
\Gamma_{\text {Bulk }}(\mathrm{SC})=\text { Non }
$$

It is well known that inducing pressure in phosphorene results in an increase in the Raman shift similar to other layered materials [161-164]. Analyses of the high-pressure Raman spectra reveal three distinct regions (Figures 3.35 - 3.37) of pressure. Up to 6.5 GPa the $A_{g}^{1}$ mode showed a linear increase with a large coefficient of $5.78\left(\mathrm{~cm}^{-1}\right)(\mathrm{GPa})^{-1}$. The $B_{2 g}$ mode initially increases by $3 \mathrm{~cm}^{-1}$ and saturates above $2 \mathrm{GPa}$. The $A_{g}^{2}$ mode does not show any significant pressure dependence. Upon further compression, we observed that the shifts in the Raman modes above $\sim 6.5 \mathrm{GPa}$ are unambiguously different than that 
of the orthorhombic phase which was interpreted as a phase transition from orthorhombic to rhombohedral crystal structure. At high pressures above $10 \mathrm{GPa}$, a new phonon modes appear at $\sim 425 \mathrm{~cm}^{-1}$ in the Raman spectra. This change can be associated to occurrence of a phase transition from orthorhombic to simple cubic in few layer black phosphorus. With further increase of the pressure above $\sim 14 \mathrm{GPa}$, the vibrational Raman modes were found to disappear. These spectral changes under high pressure can be due to nonhydrostatic behavior of DMF at that pressure. Upon decompression, the spectroscopic changes are reversible and the high-pressure phase reverts to the original structure.

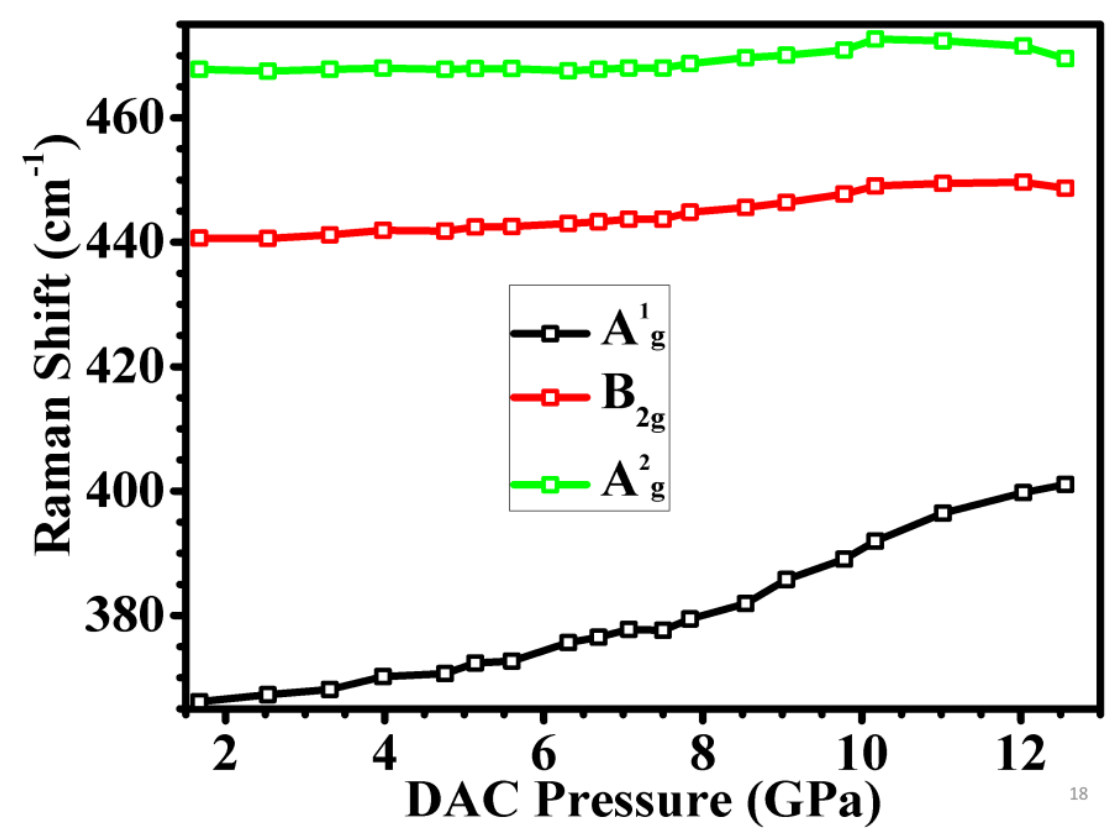

Figure 3.34 | Raman shift of three prominent peaks of phosphorene as a function of pressure. 


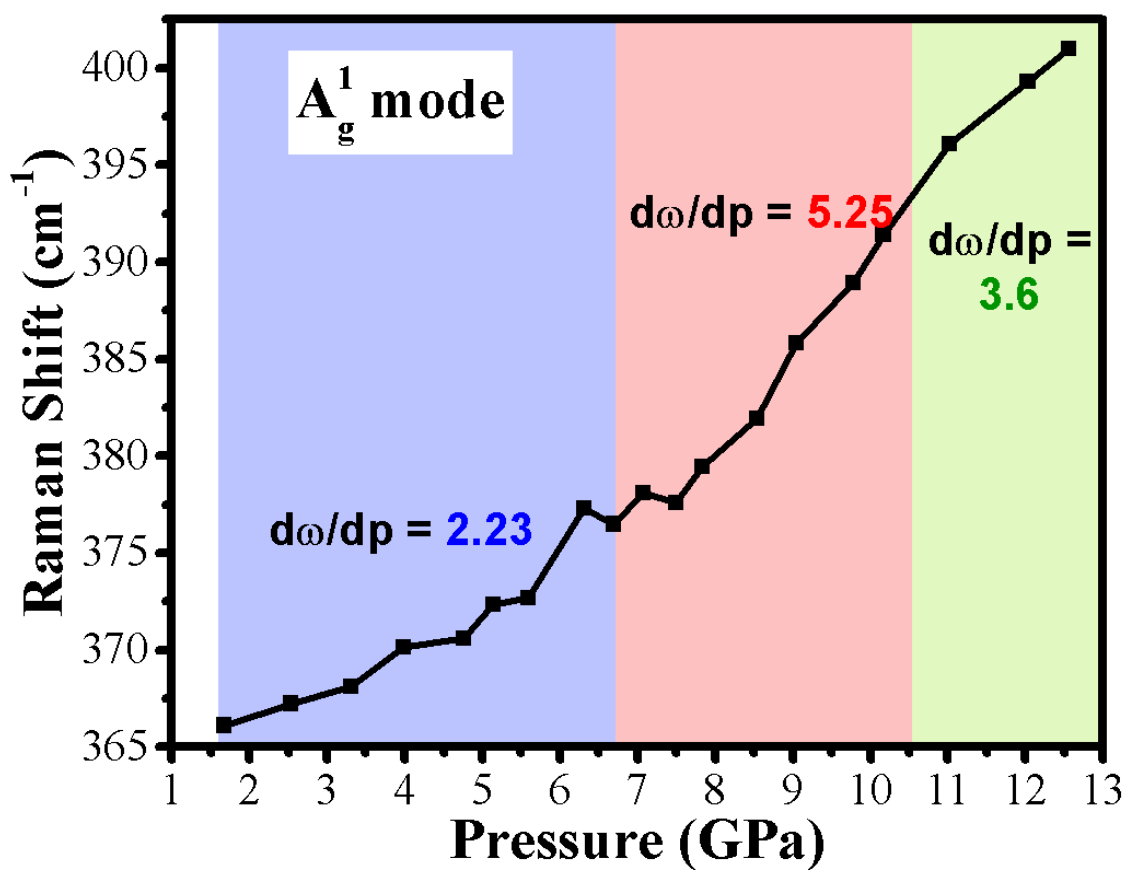

Figure 3.35 | Raman $A_{1 g}$ peak position of phosphorene vs. pressure (red) and its linear fit (black)

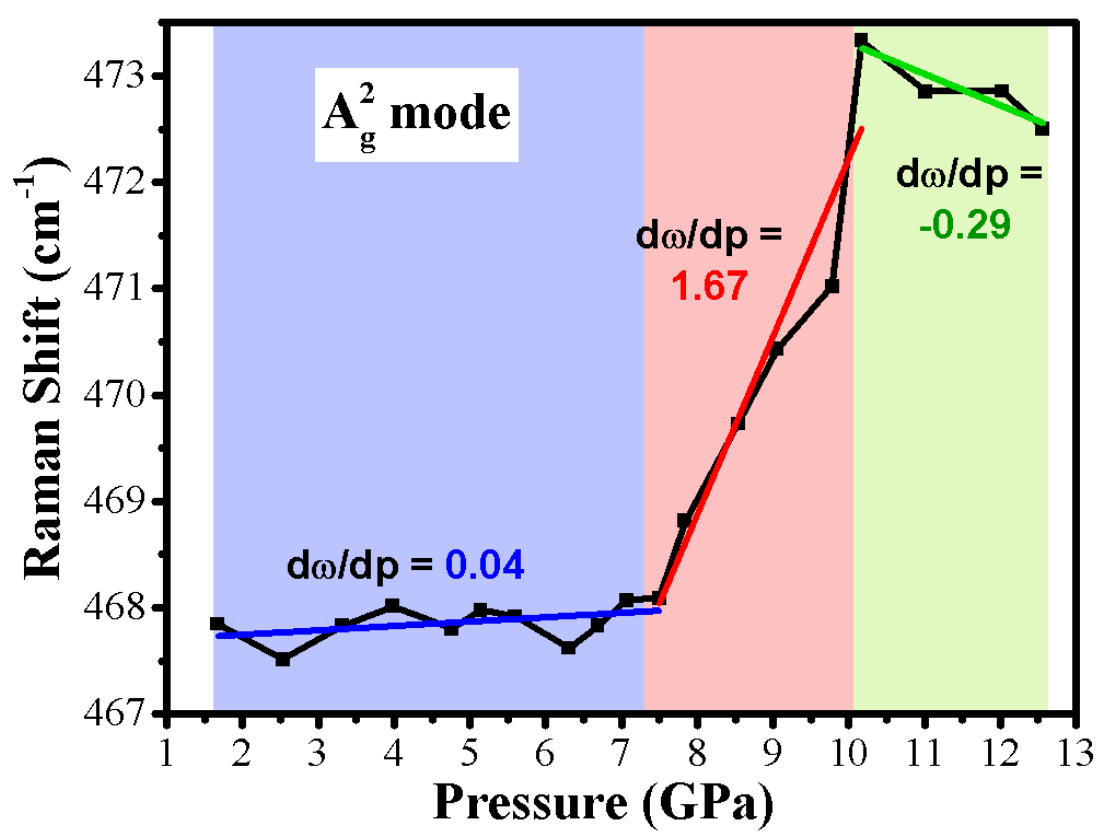

Figure 3.36 | Raman shift of $A_{2 g}$ mode of phosphorene vs. pressure (blue) and its linear fit (black) 


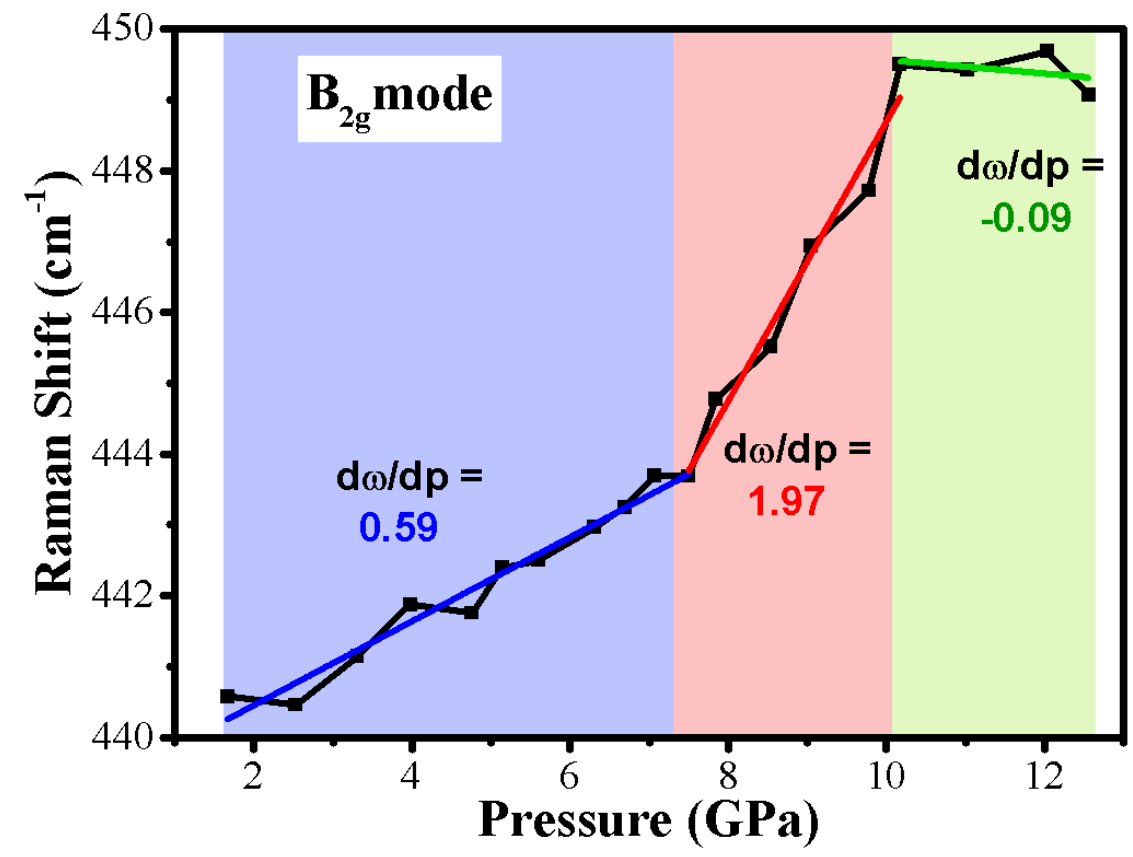

Figure 3.37 | Raman shift of $\mathrm{B}_{2 \mathrm{~g}}$ mode of phosphorene vs. pressure (green) and its linear fit (black) 


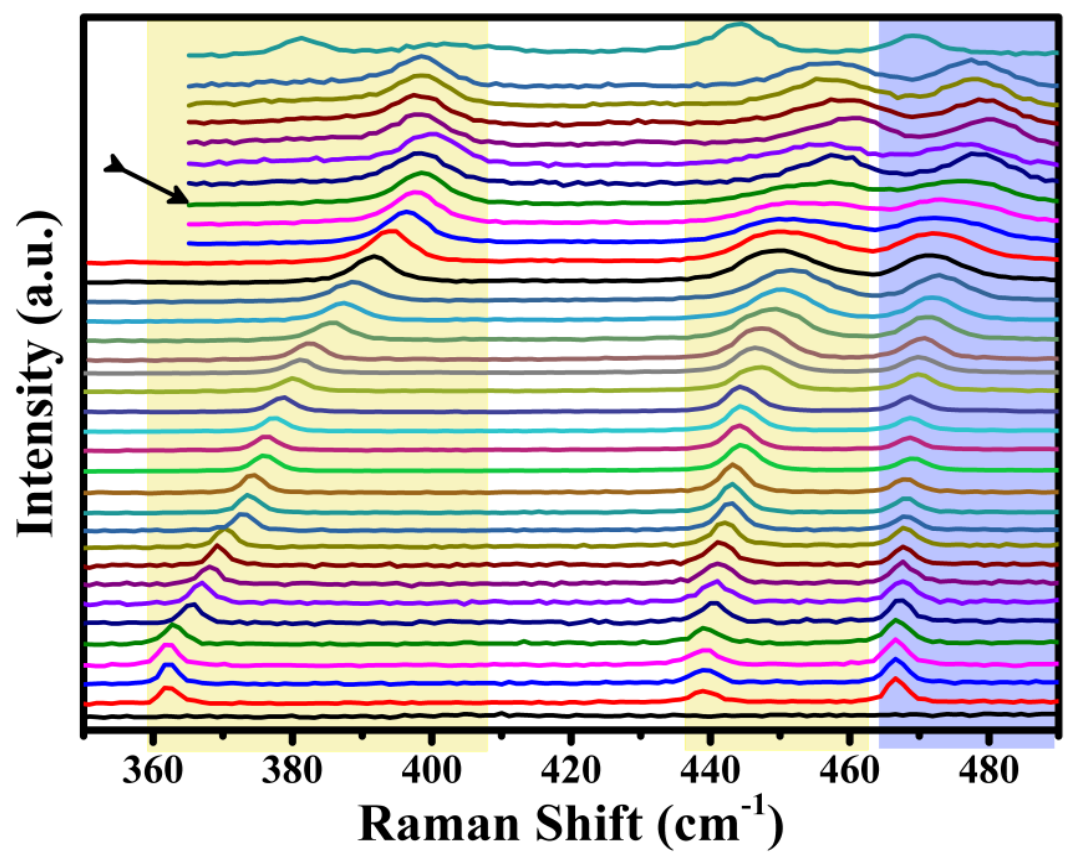

Figure 3.38 | Raman shift of a full pressure cycle of phosphorene from normal pressure (bottom spectrum) to $13 \mathrm{GPa}$ (indicated by the arrow) and returning to ambient pressure (upper one). 


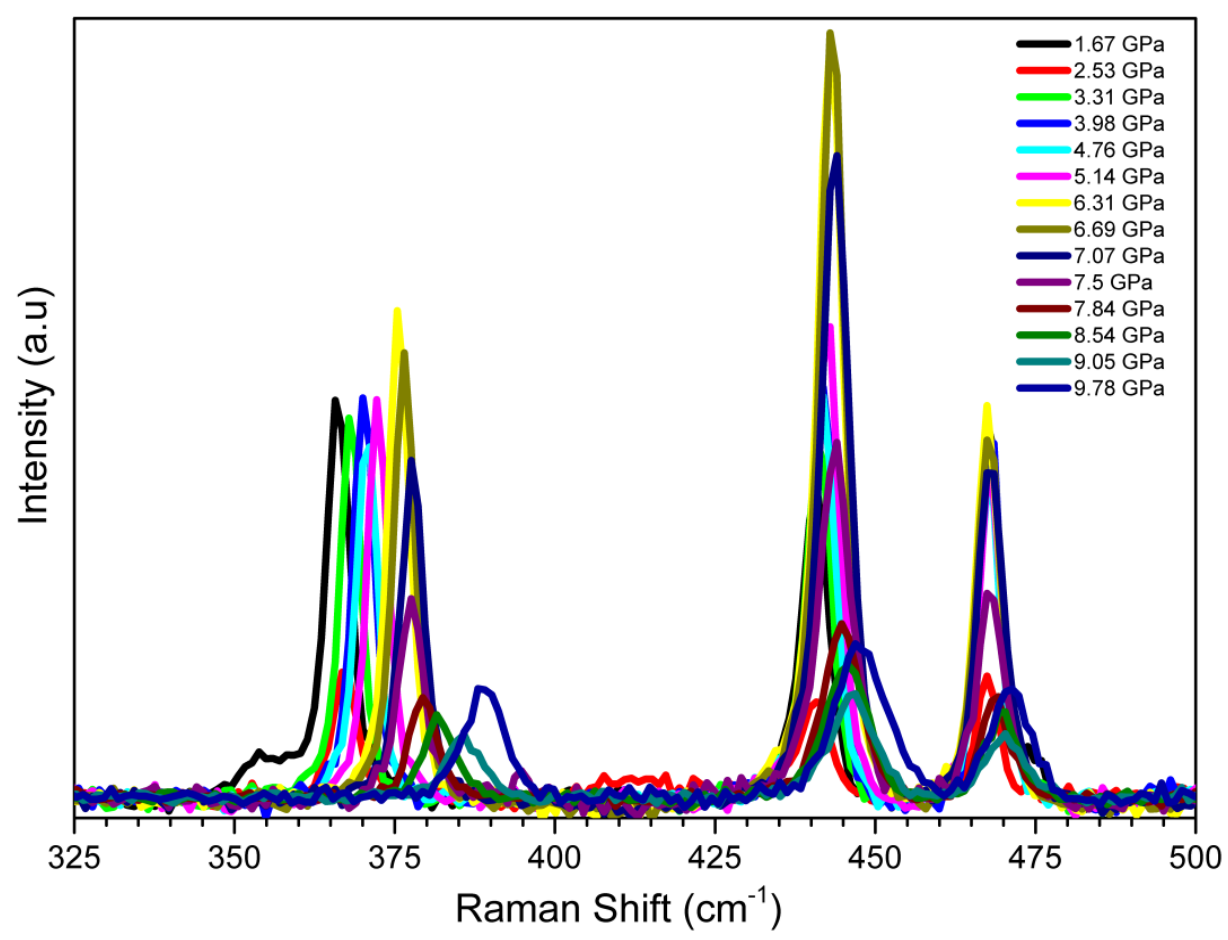

Figure 3.39 | Normalized Raman spectra of phosphorene vs. pressure

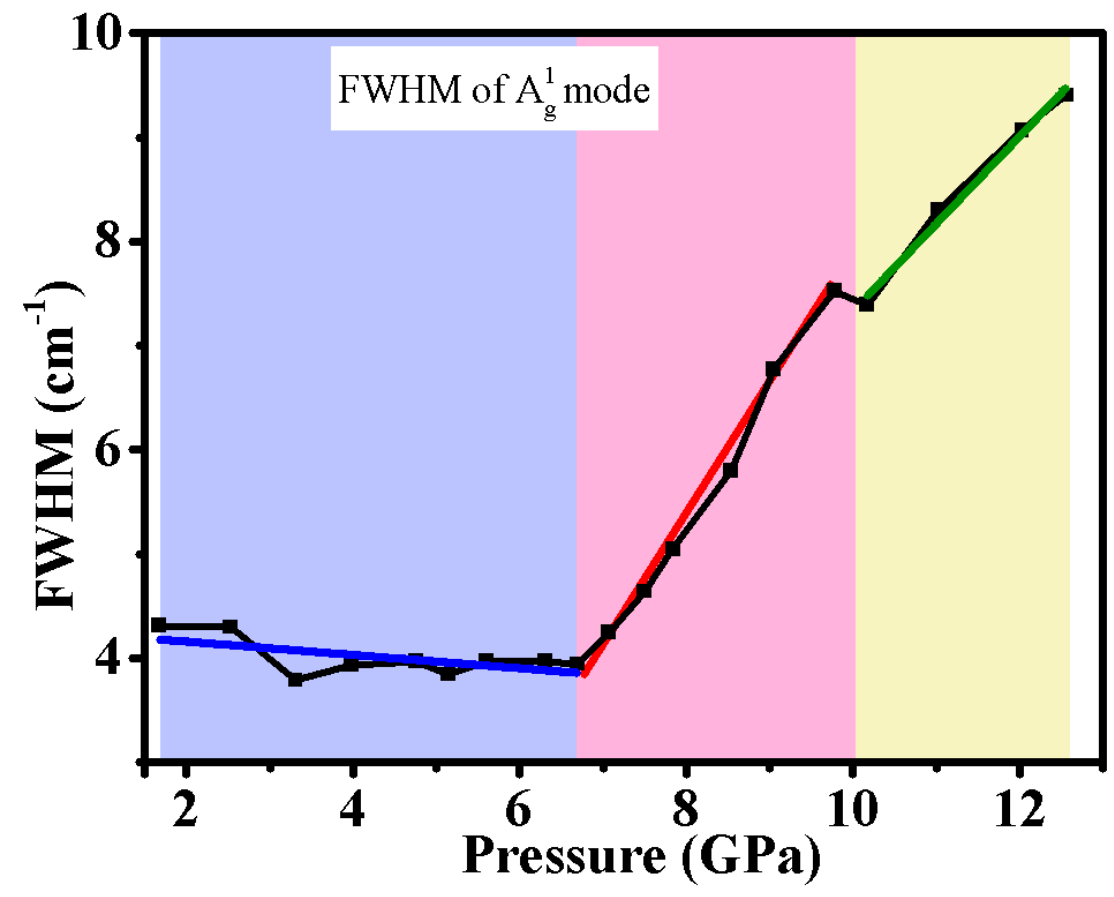

Figure 3.40 | Full-Width Half Maximum (FWHM) of $\mathrm{A}_{1 \mathrm{~g}}$ Raman mode as a function of the pressure. 


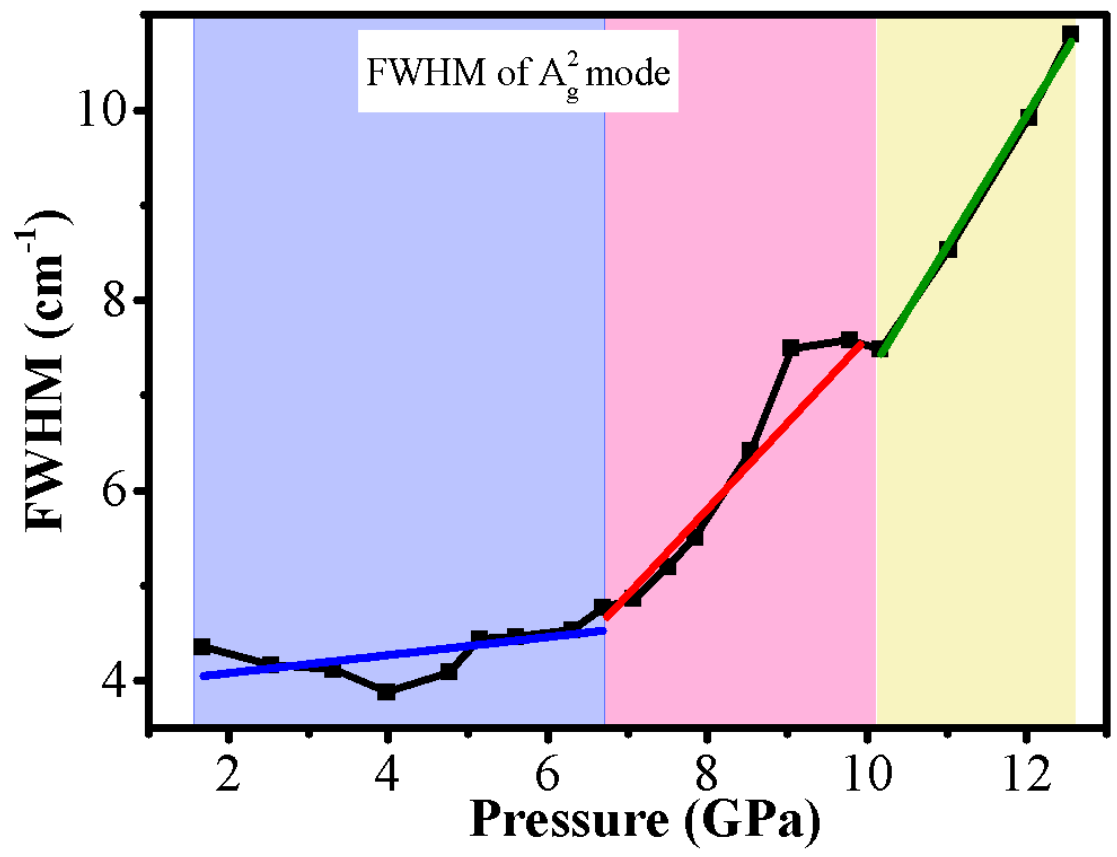

Figure 3.41 | Full-Width Half Maximum (FWHM) of $\mathrm{A}_{2 \mathrm{~g}}$ Raman mode as a function of the pressure.

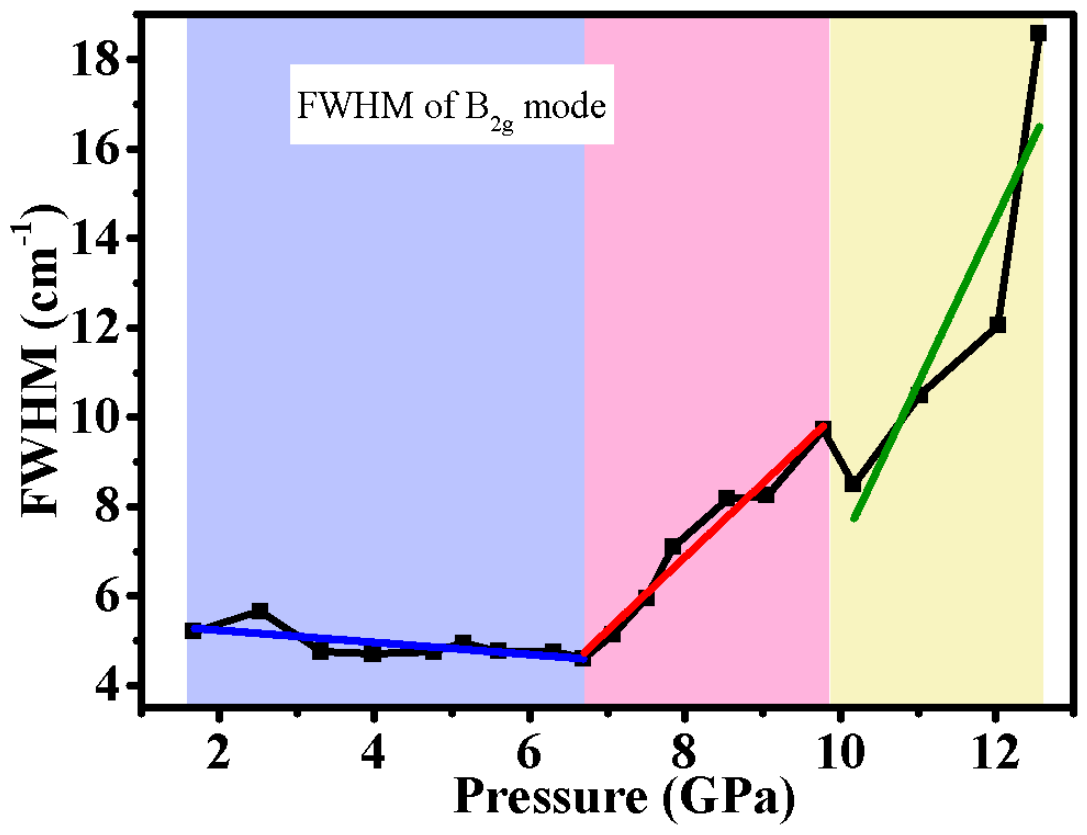

Figure 3.42 | Full-Width Half Maximum (FWHM) of $\mathrm{B}_{2 \mathrm{~g}}$ Raman mode as a function of the pressure. 
These results emphasize the prominent role of pressure in modifying the vibrational and electronic properties of two-dimensional systems. In conclusion, the group theory analysis along with the experimental Raman measurements indicated the pressureinduced structural phase transitions in few-layer BP. High-pressure Raman results suggest existence of two pressure-induced structural phase transitions in few-layer BP samples, which is drastically different from the transformation sequence of bulk BP. 


\section{CHAPTER 4: SUMMARY AND OUTLOOK}

The synthesis of materials under high pressure is a vast area of physics, chemistry, and engineering. High-pressure technology is frequently being used in materials science to improve the electronic and atomic structure of materials and tune various materials properties. Besides, it is also being used in solid-state chemistry to modify existing materials and to synthesize non-equilibrium phases or novel compounds. High pressure can severely reduce the inter-atomic volume and result in densification effects or enhancement of the reactivity. One of the advantages of high-pressure approaches in synthesis chemicals is the fact that these are in general cleaner and faster technique than purely chemical routes, which normally leads to the formation of by-products, or results in undesired disorder or phase separation.

Encouraged by advantageous of high-pressure synthesis method, we designed and demonstrated the successful synthesis of $\alpha-\mathrm{AgGaO}_{2}$ for the first time via solid-state reaction. In this project, high pressure was used to stabilize $\mathrm{Ag}_{2} \mathrm{O}$ and to drive a direct synthesis of rhombohedral silver gallium oxide $\left(\mathrm{AgGaO}_{2}\right)$ through a solid-state reaction between $\mathrm{Ag}_{2} \mathrm{O}$ and $\mathrm{Ga}_{2} \mathrm{O}_{3}$ powders. The instability of silver oxide under the normal conditions imposes considerable challenges for the preparation of silver-gallium oxide

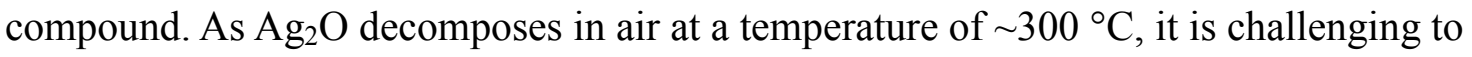
synthesize $\mathrm{AgGaO}_{2}$ in a one-step via a conventional solid-state reaction technique in an open system. Synthesis conditions of high temperature and, more importantly, of high 
pressure was essential to facilitate the synthesis reaction. A thorough characterization of the synthesized sample was performed, and the rhombohedral crystal phase was identified explicitly. Furthermore, vibrational phonon modes analysis indicates good agreement between theoretical DFT calculations and experimental Raman data. In addition, using the DFT/GGA $+U$ formalism, we found the bandgap of $1.83 \mathrm{eV}$ theoretically, which was much closer to the experimental data, than calculated values has been reported previously.

Phosphorene was synthesized in 2014 for the first time, and since then it has been the focus of numerous research projects. This huge research attraction is due to the exceptional and intriguing properties of phosphorene. Its widely tunable band gap, strong in-plane anisotropy, and high carrier mobility make phosphorene the center of various fundamental studies. Recently, numerous theoretical studies have been conducted to investigate strain engineering to additionally tune the electronic structure of phosphorene and few-layer BP. Although several theoretical studies have predicted an electronic and structural phase transition in few-layer BP, there is not experimental study to indicate the transition.

In order to understand the effect of strain on structural, vibrational and electronic properties of few-layer black phosphorus (BP) and to verify the theoretical study with experimental data we applied high pressure up to $\sim 14 \mathrm{GPa}$ to the samples using diamond anvil cell. , Raman scattering data along with the group theory information was thoroughly investigated. We described and interpreted the evolution of vibrational modes of black phosphorene under high pressure. It was found that by increasing the pressure, all three prominent Raman peaks of orthorhombic few-layer BP underwent a blue shift, 
but with different rates. The two $B_{2 g}$ and $A_{g}^{2}$ modes showed little pressure dependence. In contrast the $A_{g}^{1}$ modes showed strong pressure dependence. These behavior can be explained by the fact that $B_{2 g}$ and $A_{g}^{2}$ modes are related to deformation of the in plane atomic arrangement with strong bonding forces but $A_{g}^{1}$ modes correspond to out of plane weak van der Waals forces which are severely impacted by high pressure. Further Raman data analysis revealed existence of three distinct pressure regions signifying the crystal phase transition of the sample. Full Width Half Maximum (FWHM) of Raman peaks showed only a small decrease within the pressure range of zero to $\sim 6.5 \mathrm{GPa}$ while it increased significantly within the pressure ranges of 6.5 to 10 and 10 to $13.5 \mathrm{GPa}$, but with different rates.

For years, graphene and its derivatives have been explored intensively and remain in the center of attention in the field of materials science due to its extraordinary properties. Combinations of the tremendously large surface area, elegant electrical and thermal conductivity, superior chemical stability, and extreme mechanical strength make graphene an essential component for fabricating functional materials for a broad range of applications. However, practical limitations such as the difficulty of mass production of high-quality samples render a barrier to extensive implementation of graphene in practical devices. For example, during the processing of graphene into an actual device, the strong $\pi-\pi$ interaction between graphene sheets cause the isolated graphene flakes to restack to form graphite-like powders which can substantially reduce the specific surface area and thereby result in inefficient utilization of the graphene layers. 
To address these issues and to make full utilization of the high intrinsic surface area of graphene we developed and established a simple, inexpensive, and highly scalable method to grow graphene in the form of a three-dimensional network architecture. The technique is based on pyrolysis of organic materials, and it is almost identical to the conventional chemical vapor deposition (CVD) process except for use of solid organic sources of carbon instead of methane or other gas sources. We found that this method to be simpler, faster and more efficient compared to conventional CVD process. Furthermore, three-dimensional graphene-based materials offer an easy and versatile platform for functionalization and integration into devices. Accordingly, 3D graphene materials and their derivatives triggered extensive studies for numerous potential applications in energy, electrochemistry, bioelectrochemistry and pollution management. High pore volume and the large surface area along with the intrinsic graphene properties, including high mechanical strength excellent electronic conductivity, and perfect thermal stability are additional advantageous of graphene 3D architectures. Several samples with various conditions before and during the growth were prepared using the pyrolysis (CVD with solid carbon source) method. The conditions variable were included surface treatments of Ni foam using different acids, annealing temperatures, the concentration of organic acids and mass flow rate of hydrogen and argon gasses. Based on post growth characterization, we found that the best quality samples of pure 3D graphene result from using of $\mathrm{HCl}$ for $\mathrm{Ni}$ surface etching, and the growth temperature of $1000^{\circ} \mathrm{C}$. In the case of nitrogen doped 3D graphene samples, 1:1 weight ratio solution of urea:citric-acid and $1000{ }^{\circ} \mathrm{C}$ growth temperature showed the highest nitrogen concentration and uniformity. The statistical analysis of Raman spectra of all samples grown at $1000{ }^{\circ} \mathrm{C}$ showed general 
uniformity and high crystallinity of multilayered graphitic shells which were also, verified by electron microscopy. The effect of nitrogen doping of 3D graphene samples on their Raman spectra was well correlated with XPS results, i.e. the intensity of Raman D-band and the N (1s) XPS spectra; both were used as an indication of nitrogen concentration in the graphene structures. We also conducted an extensive statistical analysis of Raman data to establish a correlation with the quality of samples including coverage, uniformity, the number of graphitic layers, the crystallinity of the structure and concentration of defects.

The 3D graphene samples were used for electrochemical reduction of $\mathrm{CO}_{2}$ gas. The electrochemical testing demonstrated a significant increase in catalytic activity when our synthesized 3D graphene foam was used as the core-shell of a layer of nitrogen-doped amorphous carbon. We compared catalytic activity of this combination with a nickel foam, a silver film, and a pure three-dimensional graphene foam (Figure 3.28). 


\section{REFERENCES}

1 Ackland, G. J. High-Pressure Phases of Group Iv and Iii-V Semiconductors. Reports on Progress in Physics 64, 483 (2001).

2 Schettino, V. \& Bini, R. Constraining Molecules at the Closest Approach:

Chemistry at High Pressure. Chemical Society Reviews 36, 869-880, doi:10.1039/B515964B (2007).

3 Hemley, R. J. \& Ashcroft, N. W. The Revealing Role of Pressure in the Condensed Matter Sciences. Physics Today 51, 26-32, doi:10.1063/1.882374 (1998).

4 Jayaraman, A. The Diamond-Anvil High-Pressure Cell. Scientific American 250, 54 - 62, doi:10.1038/scientificamerican0484-54 (1984).

5 Liebermann, R. C. Multi-Anvil, High Pressure Apparatus: A Half-Century of Development and Progress. High Pressure Research 31, 493-532, doi:10.1080/08957959.2011.618698 (2011).

6 Dmitrii Yu, P. Minerals of the Deep Geospheres. Physics-Uspekhi 45, 439 (2002).

7 Kim, M., Dias, R., Ohishi, Y., Matsuoka, T., Chen, J.-Y. \& Yoo, C.-S. PressureInduced Transformations of Dense Carbonyl Sulfide to Singly Bonded Amorphous Metallic Solid. Scientific Reports 6, 31594, doi:10.1038/srep31594

https://www.nature.com/articles/srep31594\#supplementary-information (2016).

8 Russell, J. H. A Pressing Matter. Physics World 19, 26 (2006). 
9 McMillan, P. F. Chemistry at High Pressure. Chemical Society Reviews 35, 855857, doi:10.1039/B610410J (2006).

10 Thompson, W. \& Hanrahan, S. Characteristics of a Cryogenic Extreme HighVacuum Chamber. Journal of Vacuum Science \& Technology 14, 643-645, doi:doi:http://dx.doi.org/10.1116/1.569168 (1977).

11 Dubrovinsky, L., Dubrovinskaia, N., Bykova, E., Bykov, M., Prakapenka, V., Prescher, C., Glazyrin, K., Liermann, H. P., Hanfland, M., Ekholm, M., Feng, Q., Pourovskii, L. V., Katsnelson, M. I., Wills, J. M. \& Abrikosov, I. A. The Most Incompressible Metal Osmium at Static Pressures above 750 Gigapascals. Nature 525, 226-229, doi:10.1038/nature14681 (2015).

12 George, V. T., Brooks, G. \& Humphrey, T. C. Regulation of Cell Cycle and Stress Responses to Hydrostatic Pressure in Fission Yeast. Molecular Biology of the Cell 18, 4168-4179, doi:10.1091/mbc.E06-12-1141 (2007).

13 Schettino, V., Bini, R., Ceppatelli, M. \& Citroni, M. Activation and Control of Chemical Reactions at Very High Pressure. Physica Scripta 78, 058104, doi:10.1088/0031-8949/78/05/058104 (2008).

14 Badding, J. V., Parker, L. J. \& Nesting, D. C. High Pressure Synthesis of Metastable Materials. Journal of Solid State Chemistry 117, 229-235, doi:http://dx.doi.org/10.1006/jssc.1995.1268 (1995).

15 Bini, R., Ceppatelli, M., Citroni, M. \& Schettino, V. From Simple to Complex and Backwards. Chemical Reactions under Very High Pressure. Chemical Physics 398, 262-268, doi:10.1016/j.chemphys.2011.04.021 (2012). 
16 Hamad R. Jappor1, M. A. A. a. A. M. A.-1. Electronic Structure of Alp under Pressure Using Semiempirical Method. doi:10.2174/1874186X01003010001 (2010).

17 Hung, V. V., Masuda-Jindo, K., Hanh, P. T. M. \& Hai, N. T. Equation of States and Melting Temperatures of Diamond Cubic and Zincblende Semiconductors: Pressure Dependence. Journal of Physics: Conference Series 98, 032001 (2008).

18 Sherman, M. S. Going to Extremes. Science \& Technology Review July/August 2004 (2004).

19 Yin, M. T. \& Cohen, M. L. Theory of Static Structural Properties, Crystal Stability, and Phase Transformations: Application to Si and Ge. Physical Review $B$ 26, 5668-5687 (1982).

20 Car, R. \& Parrinello, M. Unified Approach for Molecular Dynamics and DensityFunctional Theory. Physical Review Letters 55, 2471-2474 (1985).

21 Saib, S. \& Bouarissa, N. High-Pressure Band Parameters for Gaas: First Principles Calculations. Solid-State Electronics 50, 763-768, doi:http://doi.org/10.1016/j.sse.2006.04.002 (2006).

22 Hohenberg, P. \& Kohn, W. Inhomogeneous Electron Gas. Physical Review 136, B864-B871 (1964).

23 Kohn, W. \& Sham, L. J. Self-Consistent Equations Including Exchange and Correlation Effects. Physical Review 140, A1133-A1138 (1965).

24 Perdew, J. P. \& Zunger, A. Self-Interaction Correction to Density-Functional Approximations for Many-Electron Systems. Physical Review B 23, 5048-5079 (1981). 
25 Perdew, J. P. \& Wang, Y. Accurate and Simple Analytic Representation of the Electron-Gas Correlation Energy. Physical Review B 45, 13244-13249 (1992).

26 Ceperley, D. M. \& Alder, B. J. Ground State of the Electron Gas by a Stochastic Method. Physical Review Letters 45, 566-569 (1980).

27 Perdew, J. P., Burke, K. \& Ernzerhof, M. Generalized Gradient Approximation Made Simple. Physical Review Letters 77, 3865-3868 (1996).

28 Brazhkin, V. V. High-Pressure Synthesized Materials: Treasures and Hints. High Pressure Research 27, 333-351, doi:10.1080/08957950701546956 (2007).

29 San-Miguel, A. Nanomaterials under High-Pressure. Chem Soc Rev 35, 876-889, doi:10.1039/b517779k (2006).

30 Gérard, D. High Pressure in Solid-State Chemistry. Journal of Physics: Condensed Matter 14, 11031-11035 (2002).

31 "Fritz Haber - Biographical". Nobelprize.Org. Nobel Media Ab 2014. $<$ Http://Www.Nobelprize.Org/Nobel_Prizes/Chemistry/Laureates/1918/HaberBio.Html>.

32 "The Nobel Prize in Chemistry 1931". Nobelprize.Org. Nobel Media Ab 2014. <Http://Www.Nobelprize.Org/Nobel_Prizes/Chemistry/Laureates/1931/>, $<$ http://www.nobelprize.org/nobel_prizes/chemistry/laureates/1931/> (

33 "Percy W. Bridgman - Biographical". Nobelprize.Org. Nobel Media Ab 2014. $<$ Http://Www.Nobelprize.Org/Nobel_Prizes/Physics/Laureates/1946/BridgmanBio.Html>. 
34 Relaxation of the Structure of Simple Metal Ion Complexes in Aqueous Solutions at up to Supercritical Conditions. The Journal of Chemical Physics 118, 719-727, doi:10.1063/1.1524616 (2002).

35 Catalli, K., Shim, S.-H. \& Prakapenka, V. Thickness and Clapeyron Slope of the Post-Perovskite Boundary. Nature 462, 782-785, doi:http://www.nature.com/nature/journal/v462/n7274/suppinfo/nature08598_S1. html (2009).

36 New Developments in Laser-Heated Diamond Anvil Cell with in Situ Synchrotron X-Ray Diffraction at High Pressure Collaborative Access Team. Review of Scientific Instruments 86, 072201, doi:10.1063/1.4926895 (2015).

37 Paszkowicz, W. High-Pressure Powder X-Ray Diffraction at the Turn of the Century. Nuclear Instruments \& Methods in Physics Research Section B-Beam Interactions with Materials and Atoms 198, 142-182, doi:10.1016/s0168$583 x(02) 01129-1(2002)$.

38 Brister, K. X-Ray Diffraction and Adsorption at Extreme Pressures. Review of Scientific Instruments 68, 1629-1647, doi:10.1063/1.1147969 (1997).

39 Lange, R. \& Balny, C. Uv-Visible Derivative Spectroscopy under High Pressure. Biochimica Et Biophysica Acta-Protein Structure and Molecular Enzymology 1595, 80-93, doi:10.1016/s0167-4838(01)00336-3 (2002).

40 Boffa Ballaran, T., Kurnosov, A. \& Trots, D. Single-Crystal X-Ray Diffraction at Extreme Conditions: A Review. High Pressure Research 33, 453-465, doi:10.1080/08957959.2013.834052 (2013). 
41 Ballaran, T. B., Kurnosov, A. \& Trots, D. Single-Crystal X-Ray Diffraction at Extreme Conditions: A Review. High Pressure Research 33, 453-465, doi:10.1080/08957959.2013.834052 (2013).

42 Li, M., Gao, C.-X., Ma, Y.-Z., He, C.-Y., Hao, A.-M., Zhang, D.-M., Li, Y.-C., Liu, J. \& Wang, D.-J. Resistivity Measurement of Molten Olivine in a LaserHeated Diamond Anvil Cell. Chinese Physics Letters 24, 1010 (2007).

43 New Diamond Anvil Cell System for in Situ Resistance Measurement under Extreme Conditions. Review of Scientific Instruments 77, 123902, doi:10.1063/1.2400669 (2006).

44 Yang, J., Liu, C.-L. \& Gao, C.-X. In Situ Electrical Resistance and Activation Energy of Solid C 60 under High Pressure. Chinese Physics B 22, 096202 (2013).

45 Ménez, B., Bureau, H., Cauzid, J., Malavergne, V., Somogyi, A., Simionovici, A., Munoz, M., Avoscan, L., Rommevaux-Jestin, C. \& Gouget, B. in Modern Research and Educational Topics in Microscopy (ed J. Díaz A. Méndez-Vilas) 976 à 988 (Formatex, 2007).

46 Torchio, R., Mathon, O. \& Pascarelli, S. Xas and Xmcd Spectroscopies to Study Matter at High Pressure: Probing the Correlation between Structure and Magnetism in the 3d Metals. Coordination Chemistry Reviews 277, 80-94, doi:10.1016/j.ccr.2014.02.024 (2014).

47 Liu, A. \& Song, Y. In Situ High-Pressure and Low-Temperature Study of Ammonia Borane by Raman Spectroscopy. The Journal of Physical Chemistry C 116, 2123-2131, doi:10.1021/jp205962g (2012). 
48 Tiwari, A., Butler, I. S. \& Kozinski, J. A. A Brief Overview of the Effect of High Pressures on the Vibrational Spectra of Biomaterials. Applied Spectroscopy Reviews 44, 552-567, doi:10.1080/05704920903178421 (2009).

49 Goncharov, A. F. \& Struzhkin, V. V. Raman Spectroscopy of Metals, HighTemperature Superconductors and Related Materials under High Pressure. Journal of Raman Spectroscopy 34, 532-548, doi:10.1002/jrs.1030 (2003).

50 Edwards, G. M. \& Butler, I. S. Pressure-Tuning Spectroscopy of Inorganic Compounds: A Summary of the Past 15 Years. Coordination Chemistry Reviews 199, 1-53 (2000).

51 Lowitzer, S., Winkler, B. \& Tucker, M. Thermoelastic Behavior of Graphite from in Situ High-Pressure High-Temperature Neutron Diffraction. Physical Review B 73, 214115 (2006).

52 Nasu, S. High Pressure Mössbauer Spectroscopy Using a Diamond Anvil Cell. Hyperfine Interactions 90, 59-75, doi:10.1007/BF02069118 (1994).

$5357 \mathrm{fe}$ Mössbauer Spectroscopy in a Diamond-Anvil Cell at Variable High Pressures and Cryogenic Temperatures. Review of Scientific Instruments 65 , 3787-3792, doi:10.1063/1.1144508 (1994).

54 In Situ Impedance Measurements in Diamond Anvil Cell under High Pressure. Review of Scientific Instruments 81, 013904, doi:10.1063/1.3282444 (2010).

55 In Situ Hall Effect Measurement on Diamond Anvil Cell under High Pressure. Review of Scientific Instruments 81, 115101, doi:10.1063/1.3501384 (2010). 
56 Shimizu, H., Imaeda, H., Kume, T. \& Sasaki, S. High-Pressure Elastic Properties of Liquid and Solid Neon to \$7\Phantom $\{\backslash$ Rule $\{0.3 \mathrm{em}\}\{0 \mathrm{ex}\}\} \backslash$ Mathrm $\{\mathrm{Gpa}\} \$$. Physical Review B 71, 014108 (2005).

57 Shimizu, H. High-Pressure in Situ Brillouin Spectroscopy in a Diamond-Anvil Cell. High Pressure Research 24, 491-498, doi:10.1080/08957950412331331772 (2004).

58 Hu, Y., Kazemian, H., Rohani, S., Huang, Y. \& Song, Y. In Situ High Pressure Study of Zif-8 by Ftir Spectroscopy. Chemical Communications 47, 12694-12696, doi:10.1039/C1CC15525C (2011).

59 Wang, Z. P., Tang, X. D. \& Ding, Z. J. Raman and Photoluminescence Spectroscopy Study of Benzoic Acid at High Pressures. Journal of Physics and Chemistry of Solids 66, 895-901, doi:https://doi.org/10.1016/j.jpcs.2004.10.013 (2005).

60 Spain, I. L. Semiconductors at High Pressure: New Physics with the DiamondAnvil Cell. Contemporary Physics 28, 523-546, doi:10.1080/00107518708213742 (1987).

61 Guoyin, S. \& Ho Kwang, M. High-Pressure Studies with X-Rays Using Diamond Anvil Cells. Reports on Progress in Physics 80, 016101 (2017).

62 Bi, W., Zhao, J., Lin, J.-F., Jia, Q., Hu, M. Y., Jin, C., Ferry, R., Yang, W., Struzhkin, V. \& Alp, E. E. Nuclear Resonant Inelastic X-Ray Scattering at High Pressure and Low Temperature. Journal of Synchrotron Radiation 22, 760-765, doi:10.1107/S1600577515003586 (2015). 
63 Jacobsen, S. D., Spetzler, H., Reichmann, H. J. \& Smyth, J. R. Shear Waves in the Diamond-Anvil Cell Reveal Pressure-Induced Instability in (Mg,Fe)O.

Proceedings of the National Academy of Sciences of the United States of America 101, 5867-5871, doi:10.1073/pnas.0401564101 (2004).

64 Bassett, W. A., Reichmann, H.-J., Angel, R. J., Spetzler, H. \& Smyth, J. R. New Diamond Anvil Cells for Gigahertz Ultrasonic Interferometry and X-Ray Diffraction. American Mineralogist 85, 283-287 (2000).

65 Pressure-Enhanced Crystallization Kinetics of Amorphous Si and Ge: Implications for Point-Defect Mechanisms. Journal of Applied Physics 70, 53235345, doi:10.1063/1.350243 (1991).

66 Schettino, V., Bini, R., Ceppatelli, M., Ciabini, L. \& Citroni, M. in Advances in Chemical Physics, Vol 131 Vol. 131 Advances in Chemical Physics (ed S. A. Rice) 105-242 (2005).

67 Schettino, V. \& Bini, R. Molecules under Extreme Conditions: Chemical Reactions at High Pressure. Physical Chemistry Chemical Physics 5, 1951-1965, doi:10.1039/b301381b (2003).

68 Hemley, R. J. Effects of High Pressure on Molecules. Annual Review of Physical Chemistry 51, 763-800 (2000).

69 Sleight, A. W. New Ternary Oxides of Mercury with the Pyrochlore Structure. Inorganic Chemistry 7, 1704-1708, doi:10.1021/ic50067a003 (1968).

70 Knyrim, J. S., Friedrichs, J., Neumair, S., Roessner, F., Floredo, Y., Jakob, S., Johrendt, D., Glaum, R. \& Huppertz, H. High-Pressure Syntheses and Characterization of the Transition Metal Borates Beta- $\mathrm{Mb}_{4} \mathrm{O}_{7}\left(\mathrm{M}=\mathrm{Mn}^{2+}, \mathrm{Ni}^{2+}\right.$, 
$\left.\mathrm{Cu}^{2+}\right)$. Solid State Sciences 10, 168-176,

doi:10.1016/j.solidstatesciences.2007.09.004 (2008).

71 Fujinaka, H., Kinomura, N., Miyamota, Y., Kume, S. \& Koizumi, M. in 7th Internat. AIRAPT Confer. High Pres. Sci. Techn.1st edn 556-557 (Pergamon Press, 1980).

72 Akhtar, M., Menon, M., Sunkara, M., Sumanasekera, G., Durygin, A. \& Jasinski, J. B. High-Pressure Synthesis of Rhombohedral A-Aggao2 Via Direct Solid State Reaction. Journal of Alloys and Compounds 641, 87-92, doi:10.1016/j.jallcom.2015.04.051 (2015).

73 L'Vov, B. V. Kinetics and Mechanism of Thermal Decomposition of Silver Oxide. Thermochimica Acta 333, 13-19, doi:http://dx.doi.org/10.1016/S00406031(99)00085-4 (1999).

74 Herley, P. J. \& Prout, E. G. The Thermal Decomposition of Silver Oxide. Journal of the American Chemical Society 82, 1540-1543, doi:10.1021/ja01492a006 (1960).

75 Benton, A. F. \& Drake, L. C. Kinetics of Reaction and Adsorption in the System Silver-Oxygen. Journal of the American Chemical Society 56, 255-263, doi:10.1021/ja01317a001 (1934).

76 Gallardo, O. A. D., Moiraghi, R., Macchione, M. A., Godoy, J. A., Perez, M. A., Coronado, E. A. \& Macagno, V. A. Silver Oxide Particles/Silver Nanoparticles Interconversion: Susceptibility of Forward/Backward Reactions to the Chemical Environment at Room Temperature. Royal Society of Chemistry Advances $\mathbf{2}$, 2923-2929, doi:10.1039/C2RA01044E (2012). 
77 Maruyama, Y., Irie, H. \& Hashimoto, K. Visible Light Sensitive Photocatalyst, Delafossite Structured Alpha-Aggao(2). J. Phys. Chem. B 110, 23274-23278, doi:10.1021/jp063406s (2006).

78 Shahriari, D. Y., Erdman, N., Haug, U. T. M., Zarzyczny, M. C., Marks, L. D. \& Poeppelmeier, K. R. Direct Synthesis of Agino 2 . Journal of Physics and Chemistry of Solids 64, 1437-1441, doi:http://dx.doi.org/10.1016/S00223697(03)00115-X (2003).

79 SHANNON, R. D., ROGERS, D. B. \& PREWITT, C. T. Chemistry of Noble Metal Oxides. I. Syntheses and Properties of Abo2 Delafossite Compounds. Inorganic Chemistry 10, 713-718 (1971).

80 Nagarajan, R. \& Tomar, N. Ultrasound Assisted Ambient Temperature Synthesis of Ternary Oxide Agmo2 (M=Fe, Ga). Journal of Solid State Chemistry 182, 1283-1290, doi:10.1016/j.jssc.2009.01.043 (2009).

81 Sheets, W. C., Stampler, E. S., Bertoni, M. I., Sasaki, M., Marks, T. J., Mason, T. O. \& Poeppelmeier, K. R. Silver Delafossite Oxides. Inorg. Chem. 47, 2696-2705, doi:10.1021/ic702197h (2008).

82 Sheets, W. C., Mugnier, E., Barnabe, A., Marks, T. J. \& Poeppelmeier, K. R. Hydrothermal Synthesis of Delafossite-Type Oxides. Chem. Mater. 18, 7-20, doi:10.1021/cm051791c (2006).

83 Gao, H. \& Duan, H. 2d and 3d Graphene Materials: Preparation and Bioelectrochemical Applications. Biosensors \& Bioelectronics 65, 404-419, doi:10.1016/j.bios.2014.10.067 (2015). 
84 Fan, X., Chen, X. \& Dai, L. 3d Graphene Based Materials for Energy Storage. Current Opinion in Colloid \& Interface Science 20, 429-438, doi:10.1016/j.cocis.2015.11.005 (2015).

85 Zhou, G., Ye, Z., Shi, W., Liu, J. \& Xi, F. Applications of Three Dimensional Graphene and Its Composite Materials. Progress in Chemistry 26, 950-960, doi:10.7536/pc131250 (2014).

86 Luo, B. \& Zhi, L. Design and Construction of Three Dimensional GrapheneBased Composites for Lithium Ion Battery Applications. Energy \& Environmental Science 8, 456-477, doi:10.1039/c4ee02578d (2015).

87 Jiang, L. \& Fan, Z. Design of Advanced Porous Graphene Materials: From Graphene Nanomesh to 3d Architectures. Nanoscale 6, 1922-1945, doi:10.1039/c3nr04555b (2014).

88 Shen, Y., Fang, Q. \& Chen, B. Environmental Applications of Three-Dimensional Graphene-Based Macrostructures: Adsorption, Transformation, and Detection. Environmental Science \& Technology 49, 67-84, doi:10.1021/es504421y (2015).

89 Liu, X., Kou, Z. \& Mu, S. Porous Graphene Materials. Progress in Chemistry 27, 1566-1577, doi:10.7536/pc150508 (2015).

90 Han, S., Wu, D., Li, S., Zhang, F. \& Feng, X. Porous Graphene Materials for Advanced Electrochemical Energy Storage and Conversion Devices. Advanced Materials 26, 849-864, doi:10.1002/adma.201303115 (2014).

91 Xu, P., Yang, J., Wang, K., Zhou, Z. \& Shen, P. Porous Graphene: Properties, Preparation, and Potential Applications. Chinese Science Bulletin 57, 2948-2955, doi:10.1007/s11434-012-5121-3 (2012). 
92 Yang, Z., Chabi, S., Xia, Y. \& Zhu, Y. Preparation of 3d Graphene-Based Architectures and Their Applications in Supercapacitors. Progress in Natural Science-Materials International 25, 554-562, doi:10.1016/j.pnsc.2015.11.010 (2015).

93 Zeng, M., Wang, W.-L. \& Bai, X.-D. Preparing Three-Dimensional Graphene Architectures: Review of Recent Developments. Chinese Physics B 22, doi:10.1088/1674-1056/22/9/098105 (2013).

94 Yan, Z., Yao, W., Hu, L., Liu, D., Wang, C. \& Lee, C.-S. Progress in the Preparation and Application of Three-Dimensional Graphene-Based Porous Nanocomposites. Nanoscale 7, 5563-5577, doi:10.1039/c5nr00030k (2015).

95 Zhang, X., Zhang, H., Li, C., Wang, K., Sun, X. \& Ma, Y. Recent Advances in Porous Graphene Materials for Supercapacitor Applications. Rsc Advances 4, 45862-45884, doi:10.1039/c4ra07869a (2014).

96 Xu, Y., Shi, G. \& Duan, X. Self-Assembled Three-Dimensional Graphene Macrostructures: Synthesis and Applications in Supercapacitors. Accounts of Chemical Research 48, 1666-1675, doi:10.1021/acs.accounts.5b00117 (2015).

97 Fang, Q., Shen, Y. \& Chen, B. Synthesis, Decoration and Properties of ThreeDimensional Graphene-Based Macrostructures: A Review. Chemical Engineering Journal 264, 753-771, doi:10.1016/j.cej.2014.12.001 (2015).

98 Russo, P., Hu, A. \& Compagnini, G. Synthesis, Properties and Potential Applications of Porous Graphene: A Review. Nano-Micro Letters 5, 260-273, doi:10.5101/nml.v5i4.p260-273 (2013). 
99 Wang, H., Yuan, X., Zeng, G., Wu, Y., Liu, Y., Jiang, Q. \& Gu, S. Three Dimensional Graphene Based Materials: Synthesis and Applications from Energy Storage and Conversion to Electrochemical Sensor and Environmental Remediation. Advances in Colloid and Interface Science 221, 41-59, doi:10.1016/j.cis.2015.04.005 (2015).

100 Xia, X. H., Chao, D. L., Zhang, Y. Q., Shen, Z. X. \& Fan, H. J. ThreeDimensional Graphene and Their Integrated Electrodes. Nano Today 9, 785-807, doi:10.1016/j.nantod.2014.12.001 (2014).

101 Cao, X., Yin, Z. \& Zhang, H. Three-Dimensional Graphene Materials: Preparation, Structures and Application in Supercapacitors. Energy \& Environmental Science 7, 1850-1865, doi:10.1039/c4ee00050a (2014).

102 Ma, Y. \& Chen, Y. Three-Dimensional Graphene Networks: Synthesis, Properties and Applications. National Science Review 2, 40-53, doi:10.1093/nsr/nwu072 (2015).

103 Mao, S., Lu, G. \& Chen, J. Three-Dimensional Graphene-Based Composites for Energy Applications. Nanoscale 7, 6924-6943, doi:10.1039/c4nr06609j (2015).

104 Ji, X., Zhang, X. \& Zhang, X. Three-Dimensional Graphene-Based Nanomaterials as Electrocatalysts for Oxygen Reduction Reaction. Journal of Nanomaterials, doi:10.1155/2015/357196 (2015).

105 Novoselov, K. S., Geim, A. K., Morozov, S. V., Jiang, D., Zhang, Y., Dubonos, S. V., Grigorieva, I. V. \& Firsov, A. A. Electric Field Effect in Atomically Thin Carbon Films. Science 306, 666-669, doi:10.1126/science.1102896 (2004). 
106 Eswaraiah, V., Zeng, Q., Long, Y. \& Liu, Z. Black Phosphorus Nanosheets: Synthesis, Characterization and Applications. Small 12, 3480-3502, doi:10.1002/smll.201600032 (2016).

107 Butler, S. Z., Hollen, S. M., Cao, L., Cui, Y., Gupta, J. A., Gutierrez, H. R., Heinz, T. F., Hong, S. S., Huang, J. \& Ismach, A. F. Progress, Challenges, and Opportunities in Two-Dimensional Materials Beyond Graphene. ACS Nano 7, 2898-2926 (2013).

108 Bhimanapati, G. R., Lin, Z., Meunier, V., Jung, Y., Cha, J., Das, S., Xiao, D., Son, Y., Strano, M. S. \& Cooper, V. R. Recent Advances in Two-Dimensional Materials Beyond Graphene. ACS Nano 9, 11509-11539 (2015).

109 Xu, M., Liang, T., Shi, M. \& Chen, H. Graphene-Like Two-Dimensional Materials. Chemical reviews 113, 3766-3798, doi:10.1021/cr300263a (2013).

110 Akhtar, M., Anderson, G., Zhao, R., Alruqi, A., Mroczkowska, J. E., Sumanasekera, G. \& Jasinski, J. B. Recent Advances in Synthesis, Properties, and Applications of Phosphorene. npj 2D Materials and Applications 1, 5, doi:10.1038/s41699-017-0007-5 (2017).

111 Ferrari, A. C., Bonaccorso, F., Fal'Ko, V., Novoselov, K. S., Roche, S., Bøggild, P., Borini, S., Koppens, F. H., Palermo, V. \& Pugno, N. Science and Technology Roadmap for Graphene, Related Two-Dimensional Crystals, and Hybrid Systems. Nanoscale 7, 4598-4810 (2015).

112 Guo, G.-C., Wei, X.-L., Wang, D., Luo, Y. \& Liu, L.-M. Pristine and DefectContaining Phosphorene as Promising Anode Materials for Rechargeable Li 
Batteries. Journal of Materials Chemistry A 3, 11246-11252,

doi:10.1039/C5TA01661D (2015).

113 Xia, F., Wang, H. \& Jia, Y. Rediscovering Black Phosphorus as an Anisotropic Layered Material for Optoelectronics and Electronics. Nature Communications 5 (2014).

114 Liu, H., Neal, A. T., Zhu, Z., Luo, Z., Xu, X., Tomanek, D. \& Ye, P. D. Phosphorene: An Unexplored 2d Semiconductor with a High Hole Mobility. ACS Nano 8, 4033-4041, doi:10.1021/nn501226z (2014).

115 Bridgman, P. W. Two New Modifications of Phosphorus. Journal of the American Chemical Society 36, 1344-1363, doi:10.1021/ja02184a002 (1914).

116 Rodin, A. S., Carvalho, A. \& Castro Neto, A. H. Strain-Induced Gap Modification in Black Phosphorus. Physical Review Letters 112, 176801 (2014).

117 Castellanos-Gomez, A., Vicarelli, L., Prada, E., Island, J. O., Narasimha-Acharya, K. L., Blanter, S. I., Groenendijk, D. J., Buscema, M., Steele, G. A., Alvarez, J. V., Zandbergen, H. W., Palacios, J. J. \& van der Zant, H. S. J. Isolation and Characterization of Few-Layer Black Phosphorus. 2D Materials 1, doi:10.1088/2053-1583/1/2/025001 (2014).

118 Appalakondaiah, S., Vaitheeswaran, G., Lebegue, S., Christensen, N. E. \& Svane, A. Effect of Van Der Waals Interactions on the Structural and Elastic Properties of Black Phosphorus. Physical Review B 86, doi:10.1103/PhysRevB.86.035105 (2012).

119 Dubrovinskaia, N., Dubrovinsky, L., Solopova, N. A., Abakumov, A., Turner, S., Hanfland, M., Bykova, E., Bykov, M., Prescher, C., Prakapenka, V. B., 
Petitgirard, S., Chuvashova, I., Gasharova, B., Mathis, Y.-L., Ershov, P., Snigireva, I. \& Snigirev, A. Terapascal Static Pressure Generation with Ultrahigh Yield Strength Nanodiamond. Science Advances 2, doi:10.1126/sciadv.1600341 (2016).

120 Bassett, W. Diamond Anvil Cell, 50th Birthday. International Journal of High Pressure Research 29, 163-186, doi:10.1080/08957950802597239 (2009).

121 Briggs, R. In Situ Study of Polymorphism and Melting of Metals and Compounds under Extreme Conditions of High Pressure and High Temperature Doctor of Philosophy thesis, University College London, (2012).

122 Characteristics of Silicone Fluid as a Pressure Transmitting Medium in Diamond Anvil Cells. Review of Scientific Instruments 75, 4450-4454, doi:10.1063/1.1786355 (2004).

123 Evaluation of the Hydrostaticity of a Helium-Pressure Medium with Powder XRay Diffraction Techniques. Journal of Applied Physics 89, 662-668, doi:10.1063/1.1328410 (2000).

124 Pressure Transmitting Medium for Fragile Structure or Single Crystal. Review of Scientific Instruments 38, 135-136, doi:10.1063/1.1720510 (1967).

125 Wang, Q., He, D., Peng, F., Lei, L., Xiong, L., Wang, P. \& Liu, J. Pressure Transmitting Medium-Dependent Structure Stability of Nanoanatase Tio2under High Pressure. High Pressure Research 34, 259-265, doi:10.1080/08957959.2014.892939 (2014).

126 Adams, D. M., Appleby, R. \& Sharma, S. K. Spectroscopy at Very High Pressures. X. Use of Ruby R-Lines in the Estimation of Pressure at Ambient and 
at Low Temperatures. Journal of Physics E: Scientific Instruments 9, 1140 (1976).

127 Forman, R. A., Piermarini, G. J., Barnett, J. D. \& Block, S. Pressure Measurement Made by the Utilization of Ruby Sharp-Line Luminescence. Science 176, 284285, doi:10.1126/science.176.4032.284 (1972).

128 Calibration of the Pressure Dependence of the R1 Ruby Fluorescence Line to 195 Kbar. Journal of Applied Physics 46, 2774-2780, doi:10.1063/1.321957 (1975).

129 An Optical Fluorescence System for Quantitative Pressure Measurement in the Diamond-Anvil Cell. Review of Scientific Instruments 44, 1-9, doi:10.1063/1.1685943 (1973).

130 Syassen, K. Ruby under Pressure. High Pressure Research 28, 75-126, doi:10.1080/08957950802235640 (2008).

131 Dewaele, A., Torrent, M., Loubeyre, P. \& Mezouar, M. Compression Curves of Transition Metals in the Mbar Range: Experiments and Projector AugmentedWave Calculations. Physical Review B 78, 104102 (2008).

132 Mao, H. K., Xu, J. \& Bell, P. M. Calibration of the Ruby Pressure Gauge to 800 Kbar under Quasi-Hydrostatic Conditions. Journal of Geophysical Research: Solid Earth 91, 4673-4676, doi:10.1029/JB091iB05p04673 (1986).

133 Köpf, M., Eckstein, N., Pfister, D., Grotz, C., Krüger, I., Greiwe, M., Hansen, T., Kohlmann, H. \& Nilges, T. Access and in Situ Growth of Phosphorene-Precursor Black Phosphorus. Journal of Crystal Growth 405, 6-10, doi:10.1016/j.jcrysgro.2014.07.029 (2014). 
134 Lange, S., Schmidt, P. \& Nilges, T. Au3snp7@Black Phosphorus: An Easy Access to Black Phosphorus. Inorganic Chemistry 46, 4028-4035, doi:10.1021/ic062192q (2007).

135 Nilges, T., Kersting, M. \& Pfeifer, T. A Fast Low-Pressure Transport Route to Large Black Phosphorus Single Crystals. Journal of Solid State Chemistry 181, 1707-1711, doi:10.1016/j.jssc.2008.03.008 (2008).

136 Kumar, S. \& Gupta, H. C. Ab Initio Study of Structural, Bonding, and Vibrational Properties of Agao2 (a=Ag,Cu) Delafossites. Computational and Theoretical Chemistry 977, 78-85, doi:10.1016/j.comptc.2011.09.018 (2011).

137 Ouyang, S., Kikugawa, N., Chen, D., Zou, Z. \& Ye, J. A Systematical Study on Photocatalytic Properties of $\mathrm{Agmo}_{2}(\mathrm{M}=\mathrm{Al}, \mathrm{Ga}$, in $)$ : Effects of Chemical Compositions, Crystal Structures, and Electronic Structures. Journal of Physical Chemistry C 113, 1560-1566, doi:10.1021/jp806513t (2009).

138 Mitchell, D. R. G. Difftools: Electron Diffraction Software Tools for Digitalmicrograph $^{\mathrm{TM}}$. Microscopy Research and Technique 71, 588-593, doi:10.1002/jemt.20591 (2008).

139 Rodríguez-Carvajal, J. Recent Advances in Magnetic Structure Determination by Neutron Powder Diffraction. Physica B 192, 55-69, doi:http://dx.doi.org/10.1016/0921-4526(93)90108-I (1993).

140 Andriotis, A. N., Mpourmpakis, G., Lisenkov, S., Sheetz, R. M. \& Menon, M. UCalculation of the Lsda+U Functional Using the Hybrid B3lyp and Hse Functionals. physica status solidi (b) 250, 356-363, doi:10.1002/pssb.201248215 (2013). 
141 Dudarev, S. L., Botton, G. A., Savrasov, S. Y., Humphreys, C. J. \& Sutton, A. P. Electron-Energy-Loss Spectra and the Structural Stability of Nickel Oxide:An Lsda+U Study. Physical Review B 57, 1505-1509 (1998).

142 Kresse, G. \& Joubert, D. From Ultrasoft Pseudopotentials to the Projector Augmented-Wave Method. Physical Review B 59, 1758-1775 (1999).

143 Martina, I., Wiesinger, R., Jembrih-Simbürger, D. \& Schreiner, M. Micro-Raman Characterisation of Silver Corrosion Products: Instrumental Set up and Reference Database. e-PS 9, 1-8 (2012).

144 Dong, H., Li, Z., Xu, X., Ding, Z., Wu, L., Wang, X. \& Fu, X. Visible LightInduced Photocatalytic Activity of Delafossite Agmo2 (M=Al, Ga, in) Prepared Via a Hydrothermal Method. Applied Catalysis B: Environmental 89, 551-556, doi:10.1016/j.apcatb.2009.01.018 (2009).

145 Ferrari, A. C. \& Basko, D. M. Raman Spectroscopy as a Versatile Tool for Studying the Properties of Graphene. Nat Nano 8, 235-246, doi:http://www.nature.com/nnano/journal/v8/n4/abs/nnano.2013.46.html\#supple mentary-information (2013).

146 Malard, L. M., Pimenta, M. A., Dresselhaus, G. \& Dresselhaus, M. S. Raman Spectroscopy in Graphene. Physics Reports 473, 51-87, doi:https://doi.org/10.1016/j.physrep.2009.02.003 (2009).

147 Ferrari, A. C. Raman Spectroscopy of Graphene and Graphite: Disorder, Electron-Phonon Coupling, Doping and Nonadiabatic Effects. Solid State Communications 143, 47-57, doi:https://doi.org/10.1016/j.ssc.2007.03.052 (2007). 
148 Wang, Y., Shao, Y., Matson, D. W., Li, J. \& Lin, Y. Nitrogen-Doped Graphene and Its Application in Electrochemical Biosensing. ACS Nano 4, 1790-1798, doi:10.1021/nn100315s (2010).

149 Wang, H., Maiyalagan, T. \& Wang, X. Review on Recent Progress in NitrogenDoped Graphene: Synthesis, Characterization, and Its Potential Applications. ACS Catalysis 2, 781-794, doi:10.1021/cs200652y (2012).

150 Feng, Y., Zhou, J., Du, Y., Miao, F., Duan, C. G., Wang, B. \& Wan, X. Raman Spectra of Few-Layer Phosphorene Studied from First-Principles Calculations. $J$ Phys Condens Matter 27, 185302, doi:10.1088/0953-8984/27/18/185302 (2015).

151 Carvalho, A., Wang, M., Zhu, X., Rodin, A. S., Su, H. \& Castro Neto, A. H. Phosphorene: From Theory to Applications. Nature Reviews Materials 1, 16061, doi:10.1038/natrevmats.2016.61 (2016).

152 Fei, R. \& Yang, L. Lattice Vibrational Modes and Raman Scattering Spectra of Strained Phosphorene. Applied Physics Letters 105, 083120, doi:10.1063/1.4894273 (2014).

153 Akahama, Y., Kobayashi, M. \& Kawamura, H. Raman Study of Black Phosphorus up to 13 Gpa. Solid State Communications 104, 311-315, doi:http://dx.doi.org/10.1016/S0038-1098(97)00325-6 (1997).

154 Vanderborgh, C. A. \& Schiferl, D. Raman Studies of Black Phosphorus from 0.25 to 7.7 Gpa at 15 K. Physical Review B 40, 9595-9599, doi:10.1103/PhysRevB.40.9595 (1989). 
155 Sugai, S. \& Shirotani, I. Raman and Infrared Reflection Spectroscopy in Black Phosphorus. Solid State Communications 53, 753-755, doi:http://dx.doi.org/10.1016/0038-1098(85)90213-3 (1985).

156 Pressure Dependence of the Lattice Vibration in the Orthorhombic and Rhombohedral Structures of Black Phosphorus. Journal of the Physical Society of Japan 50, 3356-3361, doi:10.1143/JPSJ.50.3356 (1981).

157 Ribeiro-Soares, J., Almeida, R. M., Cançado, L. G., Dresselhaus, M. S. \& Jorio, A. Group Theory for Structural Analysis and Lattice Vibrations in Phosphorene Systems. Physical Review B 91, doi:10.1103/PhysRevB.91.205421 (2015).

158 Wang, Y., Cong, C., Fei, R., Yang, W., Chen, Y., Cao, B., Yang, L. \& Yu, T. Remarkable Anisotropic Phonon Response in Uniaxially Strained Few-Layer Black Phosphorus. Nano Research 8, 3944-3953, doi:10.1007/s12274-015-0895-7 (2015).

159 Long, D. A. Intensities in Raman Spectra. I. A Bond Polarizability Theory. Proceedings of the Royal Society A: Mathematical, Physical and Engineering Sciences 217, 203-221, doi:10.1098/rspa.1953.0057 (1953).

160 Wu, J., Mao, N., Xie, L., Xu, H. \& Zhang, J. Identifying the Crystalline Orientation of Black Phosphorus Using Angle-Resolved Polarized Raman Spectroscopy. Angew Chem Int Ed Engl 54, 2366-2369, doi:10.1002/anie.201410108 (2015).

161 Clark, S. M., Jeon, K.-J., Chen, J.-Y. \& Yoo, C.-S. Few-Layer Graphene under High Pressure: Raman and X-Ray Diffraction Studies. Solid State Communications 154, 15-18, doi:10.1016/j.ssc.2012.10.002 (2013). 
162 Pressure and Temperature-Dependent Raman Spectra of Mos2 Film. Applied Physics Letters 109, 242101, doi:10.1063/1.4968534 (2016).

163 Nayak, A. P., Pandey, T., Voiry, D., Liu, J., Moran, S. T., Sharma, A., Tan, C., Chen, C.-H., Li, L.-J., Chhowalla, M., Lin, J.-F., Singh, A. K. \& Akinwande, D. Pressure-Dependent Optical and Vibrational Properties of Monolayer Molybdenum Disulfide. Nano Letters 15, 346-353, doi:10.1021/n15036397 (2015).

164 Raman Scattering of 2h-Mos2 at Simultaneous High Temperature and High Pressure (up to $600 \mathrm{~K}$ and $18.5 \mathrm{Gpa}$ ). AIP Advances 6, 035214, doi:10.1063/1.4944832 (2016). 


\section{CURRICULUM VITA}

NAME: $\quad$ Meysam Akhtar

ADDRESS: Department of Physics and Astronomy

102 Natural Science Building

University of Louisville

Louisville KY 40292

Linkedin.com/in/meysamakhtar

EDUCATION \& EXPERTISE:

Ph.D., Physics

University of Louisville (UofL)

$2013-2017$

M.Sc., Physics

University of Louisville (UofL)

$2010-2013$

B.Sc., Physics

Shahid Beheshti University

$2002-2007$

AWARDS:

- Recipient of "Doctoral Dissertation Completion Award”, UofL, Spring 2017 
• Recipient of "Conference Travel Fund”, UofL, 2016

- Recipient of "Graduate Research Fund", School of Interdisciplinary,

UofL, 2015

• Recipient of “John Dillon Jr Physics Fellowship”, UofL, 2012-13

\section{REFEREED JOURNALS:}

- Journal of Physics D: Applied Physics

- Journal of Physics: Condensed Matter

- Nanotechnology

PROFESSIONAL ASSOCIATIONS:

- Associate member of Institute of Physics (IOP)

- Member of American Physical Society (APS)

- Member of American Association for the Advancement of Science (AAAS)

- Member of American Chemical Society (ACS)

- Member of Materials Research Society (MRS)

\section{PUBLICATIONS:}

- Akhtar, M., Menon, M., Sunkara, M., Sumanasekera, G., Durygin, A., \& Jasinski, J., High-pressure synthesis of rhombohedral $\alpha-\mathrm{AgGaO} 2$ via direct solid state reaction, Journal of Alloys and Compounds 641 (2015) 87-92.

- Akhtar, M., Anderson, G., Zhao, R., Alruqi, A., Mroczkowska, J., Sumanasekera, G., Jasinski, J., Recent Advances in Synthesis, Properties, and Applications of Phosphorene, npj 2D Materials and Applications 1, 5 (2017). 
- Zhao, R., Jayasingha, R., Sherehiy, A., Dharmasena, R., Akhtar, M., Jasinski, J., Wu, S., Henner, V., Sumanasekera, G., In Situ Transport Measurements and Band Gap Formation of Fluorinated Graphene, J. Phys. Chem. C 119 (2015), 20150-20155.

• Ziolkowska, D., Jangam, J., Rudakov, G., Paronyan, T., Akhtar, M., Sumanasekera, G., Jasinski, J., Simple synthesis of highly uniform bilayer-carbon nanocages, Carbon 115 (2017) 617-624.

- Zhao, R., Akhtar, M., Alruqi, A., Dharmasena, R., Jasinski, J., Thantirige, R., Sumanasekera, G., Electrical Transport Properties of Graphene Grown at Low Temperature Using Plasma Enhanced Chemical Vapor Deposition, Materials Research Express (ACEPTED).

\section{PRESENTATIONS:}

- Akhtar, M., Dilip, J.J.S., Sumanasekera, G., Jasinski, J., "Pyrolysis Synthesis of Three-Dimensional Graphene Network for Energy Applications”, MRS Fall Meeting, Boston, MA (USA), Nov 29-Dec 4, 2015. (Poster)

- Akhtar, M., Pishgar, S., Sumanasekera, G., \& Jasinski, J., "In-situ Raman and PL spectroscopy of phosphorene under high-pressure", APS March Meeting, New Orleans, LA USA, March 13-17, 2017. (Talk)

- Akhtar, M., Menon, M., Sunkara, M., Sumanasekera, G., Durygin, A., \& Jasinski, J., "High-Pressure Synthesis of Rhombohedral Delafossite Structured of a-AgGaO2”, APS March Meeting, Baltimore, MD USA, March 14-18, 2016. (Talk) 